\title{
A revision of Aeschynanthus (Gesneriaceae) in Singapore and Peninsular Malaysia
}

\author{
D.J. Middleton \\ Herbarium, Singapore Botanic Gardens, National Parks Board, \\ 1 Cluny Road, Singapore 259569 \\ david_middleton@nparks.gov.sg
}

\begin{abstract}
The genus Aeschynanthus Jack is revised for Singapore and Peninsular Malaysia. Four species for Singapore and fourteen species for Peninsular Malaysia are recognised, keys to the species are given, all names are typified, and detailed descriptions of all species are provided. Conservation assessments are provided for all species. Eleven names are lectotypified here and one epitype is designated.
\end{abstract}

Keywords. Conservation assessments, Didymocarpoideae, identification key, lectotypifications

\section{Introduction}

Aeschynanthus Jack is a large and variable genus with around 160 species from Sri Lanka and India through southern China and Southeast Asia to New Guinea and the Solomon Islands (Weber, 2004; Middleton, 2007). The last complete account of the genus was by Clarke (1883) who included 64 species. More recently, regional revisions have been published for China (Wang et al., 1998), Thailand (Middleton, 2007), Cambodia, Laos and Vietnam (Middleton, 2009), and India (Bhattacharyya \& Goel, 2015). Checklists have been published for Singapore (Turner, 1993; Chong et al., 2009), Peninsular Malaysia (Turner, 1997), Myanmar (Kress et al., 2003), Sulawesi (Mendum \& Atkins, 2003), and Sumatra (Tjitrosoedirdjo et al., 2009). Several of these checklists are now rather out-of-date due to the discovery of new species and/or due to the synonymisation of names.

A general background to research on Aeschynanthus was given in Middleton (2007). For Singapore and Peninsular Malaysia the last comprehensive treatment was by Ridley (1923), in which 14 species of Aeschynanthus for the Malay Peninsula were recognised. The checklists for Singapore (Turner, 1993; Chong et al., 2009) both included four native species; the checklist for Peninsular Malaysia (Turner, 1997) included 17 species (one with two varieties).

In preparation for a revision of Gesneriaceae for the Flora of Peninsular Malaysia the genus has been revised for Peninsular Malaysia and for neighbouring Singapore. Fourteen species are recognised. All fourteen species are in Peninsular Malaysia and four of these are also in Singapore. One of the species in Singapore is presumed extinct there and the other three are considered Critically Endangered (Chong et al., 2009; Williams, 2014). 
An infrageneric classification is not followed in this paper pending a more thorough investigation of the genus using molecular sequence data to compare the morphological characters. A preliminary study by Denduangboripant et al. (2001) found that a phylogeny based on ITS data did not reveal monophyletic clades that corresponded to the existing sections.

All provisional IUCN Conservation Assessments given here, calculated using the methodology of IUCN (2012), are for the species throughout their range rather than only for Peninsular Malaysia and/or Singapore. Additional comments are given under each species when the local situation differs from the global.

\section{Morphological Characters}

A more detailed discussion of morphological characters is given in Middleton (2007) and references given therein. Here is a brief discussion of the range of characters found in the species in Singapore and Peninsular Malaysia.

All species are epiphytes or occasionally lithophytes. Species such as Aeschynanthus speciosus Hook., A fulgens Wall. ex R.Br. and A. rhododendron Ridl. are large with robust stems that arch or hang due to their own weight. Others such as Aeschynanthus albidus (Blume) Steud., A. angustifolius (Blume) Steud., A. fecundus P.Woods, A. longicaulis Wall. ex R.Br., A. longiflorus (Blume) A.DC., A. obconicus C.B.Clarke in A.DC. \& C.DC. and A. wallichii R.Br. are generally more delicate and are more pendulous, although usually not loosely hanging (as, for example, is found in A. gracilis Parish ex C.B.Clarke from Thailand to NE India). Aeschynanthus dischidiodes (Ridl.) D.J.Middleton has often been reported as growing from ants' nests in trees. Aeschynanthus pulcher (Blume) G.Don, A. radicans Jack and probably A. volubilis Jack either have the habit of the more delicate species previously mentioned, sometimes with long pendent stems, or creep over tree trunks and branches or rocks, rooting at the nodes. The erect habit of species such as Aeschynanthus andersonii C.B.Clarke and A. humilis Hemsl. from Thailand is not found in Singapore or Peninsular Malaysia.

The leaves of most species are opposite but are always in whorls of three or more in Aeschynanthus speciosus and A. angustifolius. In Aeschynanthus angustifolius the leaf blades are extraordinarily variable in shape (see discussion under that species). In the other species the leaves are generally ovate to elliptic and not particularly variable in shape and size. In most species the margins are entire but they are distinctly toothed in Aeschynanthus dischidioides, sometimes slightly so in A. angustifolius, and strongly undulate in $A$. speciosus. All species are shortly petiolate and the blades are mostly coriaceous, more rarely thinner and softer.

The structure of the inflorescence is discussed in Middleton (2007) and references therein. In Singapore and Peninsular Malaysia all species either lack a peduncle or the peduncle is so short as to appear absent. The flowers are, therefore, either axillary and solitary, axillary and appearing fasciculate, or subterminal and clustered. In all species in Singapore and Peninsular Malaysia the bracts are fairly small, simple and linear.

The calyx consists of five lobes free to the base (Aeschynanthus angustifolius, 
A. dischidiodes, A. fecundus, A. longicaulis, A. longiflorus and A. speciosus), or a very short tube with five long lobes (A. albidus), or of a narrow or somewhat widening tube that is around half or more of the length of the calyx with either obvious or obscure lobes at margin (A. fulgens, A. pulcher, A. radicans, A. rhododendron and A. volubilis), or of an open cup-shaped calyx with obscure lobes at the margin (A. obconicus and $A$. wallichii). The colour of the calyx can either be quite fixed within a species (it always appears to be red in $A$. obconicus for example) or very variable within a species (dark purple or red to green in A. pulcher for example). The calyx shape and size is very variable in a number of species.

The corolla is zygomorphic, tubular, and weakly to quite strongly curved. The limb is 2-lipped with the upper lip 2-lobed and the lower lip 3-lobed. Visitation by animals has not been observed in most species but pollination is assumed to be by birds for most, possibly all, species (see Middleton (2007) for further discussion). The corolla is red, orange, yellow or green, or a combination of these colours, in the species in Singapore and Peninsular Malaysia. Flower colour is highly diagnostic for most species although is often very subjectively recorded on herbarium specimen labels when it is described at all.

All Aeschynanthus species are strongly protandrous with the stamens withering as the style elongates and the stigma enlarges. There are four stamens in two pairs with the anthers of each pair fused at their tips. In Singapore and Peninsular Malaysia no species have all four anthers fused together as is found in Aeschynanthus chiritoides C.B.Clarke from Vietnam to NE India. The gynoecium consists of the stipe, the ovary, the style and the stigma. The stipe is short in all species but rather longer in Aeschynanthus rhododendron. The dimensions given in the descriptions below reflect the measurements made on specimens to hand. It should be borne in mind though that the absolute and relative lengths of the parts of the gynoecium are enormously variable depending on the age of the flower.

The fruit is a long and narrow capsule in all species. Dehiscence is loculicidal. The basal portion of the capsule, the stipe of the unfertilised gynoecium, lacks seeds and is generally short except in Aeschynanthus rhododendron where it forms an obvious narrow stalk.

The seeds have been the principal source of characters for earlier infrageneric classifications of Aeschynanthus (see Middleton (2007) and references cited therein). The seeds of Aeschynanthus species consist of the seed grain, one apical appendage and one or more hilar appendages. Seeds with two hilar appendages are not found anywhere in Malesia (Mendum et al., 2001). The apical appendage points towards the base of the capsule. In Singapore and Peninsular Malaysia there is one hilar appendage in Aeschynanthus angustifolius, A. fulgens, A. longiflorus, A. obconicus, A. pulcher, A. radicans, $A$. rhododendron, $A$. speciosus, $A$. volubilis and $A$. wallichii and three or more hilar appendages in A. albidus, A. dischidioides, A. fecundus and A. longicaulis. Of those with only one hilar appendage, the appendage is long and filiform in all species except Aeschynanthus rhododendron where it is short and stout. In a number of species there is a curious cluster of inflated cells, termed bubble cells, at the hilar end of the seed. These are found in A. obconicus, A. pulcher, A. radicans, A. volubilis and $A$. wallichii. 


\section{Materials studied}

Herbarium material was studied from the following herbaria: A, AAU, BISH, BKF, BM, C, CGE, E, FI, G, G-DC, K, KEP, KLU, K-W, L, LAE, M, MEL, MICH, NY, P, PSU, SING, SINU, TI, U, UKMB, US (herbarium codes from Thiers (continuously updated)). All specimens cited have been seen unless otherwise indicated with $n v$. A single standardised name is given for those collectors whose name appears in more than one form, including when abbreviated to initials, on different collections.

The dimensions given in the descriptions are for dried material for vegetative characters and rehydrated or fresh material for floral characters. Dimensions given closely resemble those given in Middleton (2007) for some of the taxa due to the paucity of material collected since and because Malaysian material was used in the Thai descriptions when the Thai material was insufficient (as it was for several taxa).

The vegetation types given in the Habitat and Ecology sections below follow notes on the specimens and observations in the field using the vegetation classification of Saw (2010).

\section{Aeschynanthus Jack}

Trans. Linn. Soc. London 14: 42 (1823); C.B.Clarke in A.DC. \& C.DC., Monogr. Phan. 5(1): 18 (1883); Ridley, Fl. Malay Penins. 2: 496 (1923); Wang, Fl. Reipubl. Popularis Sin. 69: 498 (1990); Middleton, Edinburgh J. Botany 64: 368 (2007); Middleton, Edinburgh J. Botany 66: 393 (2009). - TYPE: Aeschynanthus volubilis Jack.

Trichosporum D.Don, Edinburgh Philos. J. 7: 82 (1822), nom. rej; Blume, Bijdr. Fl. Ned. Ind. (1826). - TYPE: Trichosporum parviflorum D.Don (= Aeschynanthus parviflorus (D.Don) Spreng.), lectotype designated by Middleton (2007).

Rheitrophyllum Hassk., Flora 25 (2): beibl. 56 (1842). - TYPE: Rheitrophyllum subverticillatum Hassk. (= Aeschynanthus angustifolius (Blume) Steud.).

Oxychlamys Schltr., Bot. Jahrb. Syst. 58: 286 (1923). - TYPE: Oxychlamys pullei Schltr. (= Aeschynanthus oxychlamys Mendum)

Euthamnus Schltr., Bot. Jahrb. Syst. 58: 284 (1923). - TYPE: Euthamnus papuanus Schltr. (= Aeschynanthus papuanus (Schltr.) B.L.Burtt)

Micraeschynanthus Ridl., Fl. Malay Penin. 5: 324 (1925). - TYPE: Micraeschynanthus dischidioides Ridl. (= Aeschynanthus dischidioides (Ridl.) D.J.Middleton)

Epiphytic herbs or subshrubs with erect, arching or pendulous stems, these sometimes rooting along their lengths when in contact with a suitable substrate. Leaves opposite or verticillate, pedicellate; blades coriaceous to distinctly fleshy, more rarely herbaceous, 
simple, margins entire to weakly crenate or weakly dentate, sometimes somewhat undulate, venation pinnate but more often than not obscure. Inflorescence an axillary few-flowered cyme, or flowers solitary in the axils of leaves, or a pseudoterminal cluster. Flowers strongly protandrous. Calyx of 5 sepals, these free or variously fused into a tube for part or most of length, when fused the whole tubular or cup-shaped. Corolla zygomorphic, tubular, widening towards lobes, curved to various degrees, sometimes distinctly inflated at the base, glabrous to variously pubescent outside and inside; with 5 lobes, these consisting of a 2-lobed upper lip, 2 lateral lobes and a lower lobe; very variable in colour but most frequently red, orange, yellow or green (or combination of these) and then often with other darker or lighter patterning. Stamens 4 , in 2 pairs, attached to the inside of the corolla tube and occupying the space in the upper curve of the flowers, included or exserted from corolla tube when mature; vestigial staminode present; anthers of each pair attached by their apices (occasionally all 4 attached together outside this region). Disk present, annular to dentate. Pistil developing as filaments wither and reflex downwards and also occupying the space in the upper curve of the corolla tube, consisting of a sterile stipe at the base, the fertile ovary section, the style and the peltate stigma; ovules many, anatropous. Fruit a long narrow capsule which opens loculicidally by two valves. Seeds many, tiny, with short to long appendages at both ends.

About 160 species from India and southern China through Southeast Asia and Malesia to the Solomon Islands. Fourteen species in Peninsular Malaysia, four species in Singapore (of which one is considered to be nationally extinct).

Key to Aeschynanthus species recorded from Singapore

1a. Corolla predominantly green or yellowish, inside with coarse multicellular hairs; seeds with many hairs at one end 1. A. albidus

1b. Corolla red, inside without coarse multicellular hairs; seeds with only 1 hair at each end

2a. Calyx $<7 \mathrm{~mm}$ long, in a wide and shallow cup or saucer, much wider at apex than at base 14. A. wallichii

2b. Calyx $>10 \mathrm{~mm}$ long, tube mostly parallel to corolla tube, apex not much wider than base

3a. Ovary, stipe and style densely pubescent; leaves pubescent beneath

10. A. radicans

3b. Ovary with sessile glands, only stipe and style pubescent; leaves usually glabrous, more rarely sparsely pubescent beneath 9. A. pulcher 
Key to Aeschynanthus species recorded from Peninsular Malaysia

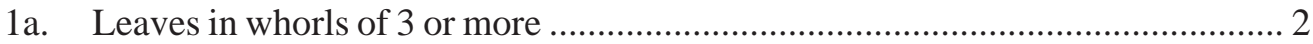

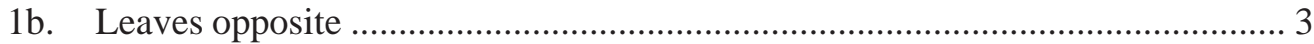

2a. Corolla predominantly green to yellow, 18-25 mm long; leaves extremely variable from ovate to linear, 1.7-43 times as long as wide

2. A. angustifolius

2b. Corolla predominantly red and orange, 54-118 mm long; leaves ovate to elliptic, never linear, 2.3-6.1 times as long as wide 12. A. speciosus

3a. Inside of corolla tube with dense multicellular hairs towards base, often in tufts; leaves sometimes conspicuously variegated; corolla at least in part green, yellow or yellowish green; seeds with $>2$ hairs at hilar end 4

3b. Inside of corolla tube without dense multicellular hairs towards base; leaves not conspicuously variegated; corolla entirely red or orange, red/orange and yellow, or a combination of colours but never green; seeds with 1 hair at hilar end ...... 7

4a. Calyx fused into a short tube at base 1. A. albidus

4b. Calyx lobes free to base 5

5a. Leaves not variegated, 1-2.4 times as long as wide, margin often distinctly dentate 3. A. dischidioides

5b. Leaves variegated, 1.7-9 times as long as wide, margin entire or very minutely and obscurely dentate 6

6a. Corolla predominantly green, 20.5-31 mm long; stamens exserted from corolla tube 7. A. longicaulis

6b. Corolla red in upper third and on lobes, 14.5-19 mm long; stamens not exserted from corolla tube 4. A. fecundus

7a. Calyx lobes free to base 6. A. longiflorus

7b. Calyx fused into a tube for part or most of length 8

8a. Calyx in a wide cup, very much wider at apex than at base, lobes often barely discernable but if obvious then much wider than long; seeds with bubble cells ....

8b. Calyx tube clasping corolla tube or only gently flaring from base, lobes usually easily discernable, either wider than long or longer than wide; seeds with or without bubble cells 10

9a. Calyx red, 8-19 mm long 8. A. obconicus

9b. Calyx green, 2.5-6.5 mm long 14. A. wallichii 
10a. Calyx lobes usually longer than wide, apex acute to acuminate (tips of lobes sometimes rounded); seeds without bubble cells ......................................... 11

10b. Calyx lobes usually wider than long, apex rounded; seeds with bubble cells ... 12

11a. Stamens not or barely exserted from corolla tube; corolla lobes spreading or reflexed 11. A. rhododendron

11b. Stamens strongly exserted from corolla tube; corolla lobes not reflexed, occasionally slightly spreading 5. A. fulgens

12a. Corolla 19.5-27 mm long 13. A. volubilis

12b. Corolla 42-66 mm long 13

13a. Ovary, stipe and style densely pubescent; leaves sparsely to densely puberulent beneath 10. A. radicans

13b. Ovary with sessile glands, only stipe and style pubescent; leaves usually glabrous, more rarely sparsely puberulent beneath 9. A. pulcher

1. Aeschynanthus albidus (Blume) Steud., Nomencl. Bot. ed. 2 1: 32 (1840); A.DC., Prod. 9: 262 (1845); Bakhuizen van den Brink, Blumea 6: 395 (1950); Backer \& Bakhuizen van den Brink, Fl. Java 2: 523 (1965); Burtt \& Woods, Notes Roy. Bot. Gard. Edinburgh 33: 479 (1975); Turner, Gard. Bull. Singapore 47: 243 (1997 ['1995’]); Lok \& Tan, Nat. Singapore 1: 5 (2008). - Bignonia albida Blume, Verh. Batav. Genootsch. Kunsten 9: 195 (1823). - Trichosporum albidum (Blume) Nees, Flora 8: 144 (1825). - Lysionotus albidus (Blume) Blume, Bijdr. Fl. Ned. Ind. 765 (1826). - Aeschynanthus purpurascens Hassk., Cat. Hort. Bot. Bogor. 154 (1844), nom. illegit; Hooker, Bot. Mag. 72: T.4236 (1846); Miquel, Fl. Ned. Ind. 2: 717 (1858); C.B.Clarke in A.DC. \& C.DC., Monogr. Phan. 5(1): 37 (1883); Ridley, Fl. Mal. Pen. 2: 497 (1923); Henderson, Malay. Wild. Fl. Dicot. 340 (1959); Turner, Gard. Bull. Singapore 45: 92 (1993). - TYPE: Indonesia, Java, Jawa Barat, Gunung Salak, Blume, C.L. s.n. (lectotype L [L0003309], designated here). (Fig. 1, 2)

?Aeschynanthus atropurpureus Van Houtte, Hort. Vanhoutt. 1(2): 42 (1846); Miquel, Fl. Ned. Ind. 2: 718 (1858). - TYPE: Not known. No original material known but Miquel's later description is specific enough to identify the taxon and it has long been placed in synonymy of Aeschynanthus albidus by other authors.

Aeschynanthus discolor T.Moore, Paxton’s Fl. Gard. 3: 55 (1852). - TYPE: Not found. Synonymy based on detailed description given in protologue.

Aeschynanthus motleyi C.B.Clarke in A.DC. \& C.DC., Monogr. Phan. 5(1): 20 (1883); Ridley, J. Linn. Soc. Bot. 32: 500 (1896); Ridley, J. Straits Branch Roy. Asiat. Soc. 44: 12 (1905); Ridley, J. Asiat. Soc. Bengal, Pt. 2, Nat. Hist. 74(2): 732 (1909). Trichosporum motleyi (C.B.Clarke) Kuntze, Revis. Gen. Pl. 478 (1891). - TYPE: 
Indonesia, Kalimantan, Kalimantan Selatan, Banjarmasin, Motley, J. 916 (lectotype K [K000831891], effectively designated by Burtt \& Woods (1975)).

Aeschynanthus motleyi var. sumatrensis C.B.Clarke in A.DC. \& C.DC., Monogr. Phan. 5(1): 20 (1883). - TYPE: Indonesia, Sumatra, Sumatera Barat, Padang, Ayer Mancior, Beccari, O. 823 (lectotype K [K000831892], designated here; isolectotypes BM [BM000537118], FI [FI013073], K [K000831893], L [L0281671], MEL).

Aeschynanthus fraserianus Kraenzl., J. Linn. Soc. Bot. 37: 284 (1906). - Trichosporum fraserianum (Kraenzl.) Merr., J. Straits Branch Roy. Asiat. Soc. special number: 530 (1921). - TYPE: Indonesia, Kalimantan, Marisinsing, Fraser 268 (holotype K [K000831890]).

Epiphyte with stems upright, arching or pendulous; stems green flushed purple, glabrous. Leaves opposite; petiole 3-13 mm long, purple, glabrous; blade slightly fleshy or coriaceous, obovate, elliptic or ovate, above green and sometimes with paler green or yellow mottling, beneath green with purple-red mottling or completely purple-red, 2.3-13.7 × 1.1-5.5 cm, 1.5-9.4 times as long as wide, apex acuminate, base rounded to cuneate, glabrous above and beneath, margin very weakly crenate, c. 3 pairs of secondary veins, obscure or weakly visible, tertiary venation obscure. Inflorescence subterminal or axillary, 1-5-flowered; peduncle c. $1 \mathrm{~mm}$ long; pedicels 7-10 mm long, glabrous. Calyx with a short tube at base, lobes free, tube clasping corolla tube at base, often slightly narrower at apex, green, green flushed with purple or red or entirely reddish, glabrous or sparsely eglandular puberulent, total length 14-30 mm long; tube 2-8.5 mm long which is $14-39 \%$ of total length, 3-6.5 mm wide at top of tube; lobes linear or narrowly triangular, slightly spreading or erect, 10.5-22 × 1-3.5 mm, apex acuminate. Corolla 16-29 mm long, tube broad at base, slightly curved, externally green or greenish yellow, lobes green or green with faint purple central lines, internally green, lobes green with red speckling or with dark purple chevrons and lines; upper lobes orbicular, not spreading or reflexed, 2.2-3.1 $\times 2.6-3.5 \mathrm{~mm}$, sinus $2.5-3 \mathrm{~mm}$ deep, apex rounded; lateral lobes orbicular, slightly spreading or not, 2.1-2.9 × 2.9-4.5 mm, apex rounded; lower lobe ovate or orbicular, slightly spreading, 2.7-3.5 × 2.5-2.8 mm, apex rounded; glabrous except for ciliate lobes, inside with five tufts of multicellular hairs near base, sometimes with some additional multicellular hairs higher up, sessile glands present at top of tube. Stamens long exserted, fused in 2 pairs; filaments cream or green, glandular pubescent, anthers pink; anterior filaments inserted at 9-14.5 mm from corolla base which is $48-53 \%$ of corolla length, filaments $21.5-23.5 \mathrm{~mm}$ long, anthers $2.7-3.2 \times 0.8-1.3 \mathrm{~mm}$; posterior filaments inserted at $9.5-14 \mathrm{~mm}$ from corolla base which is $50-58 \%$ of corolla length, filaments $19-20.5 \mathrm{~mm}$ long, anthers $1.2-2.3$ $\times$ 0.7-1.1 mm; staminode 0.7-1.6 mm long. Disk 1.2-1.5 mm high, 5-crenate. Pistil 16-26 mm long; stipe c. 2-3 mm long, with sessile glands; ovary 7-12 mm long, with sessile glands; style cream or green, 8-12 mm long, glandular puberulent; stigma pale red, 2 mm across. Capsule 11.5-40 cm long, 2.5-4 mm wide. Seed grain $1.5-2.4 \times$ 0.4-0.5 mm, warty, bubble cells absent; apical appendage a filiform hair, $13-21 \mathrm{~mm}$ 

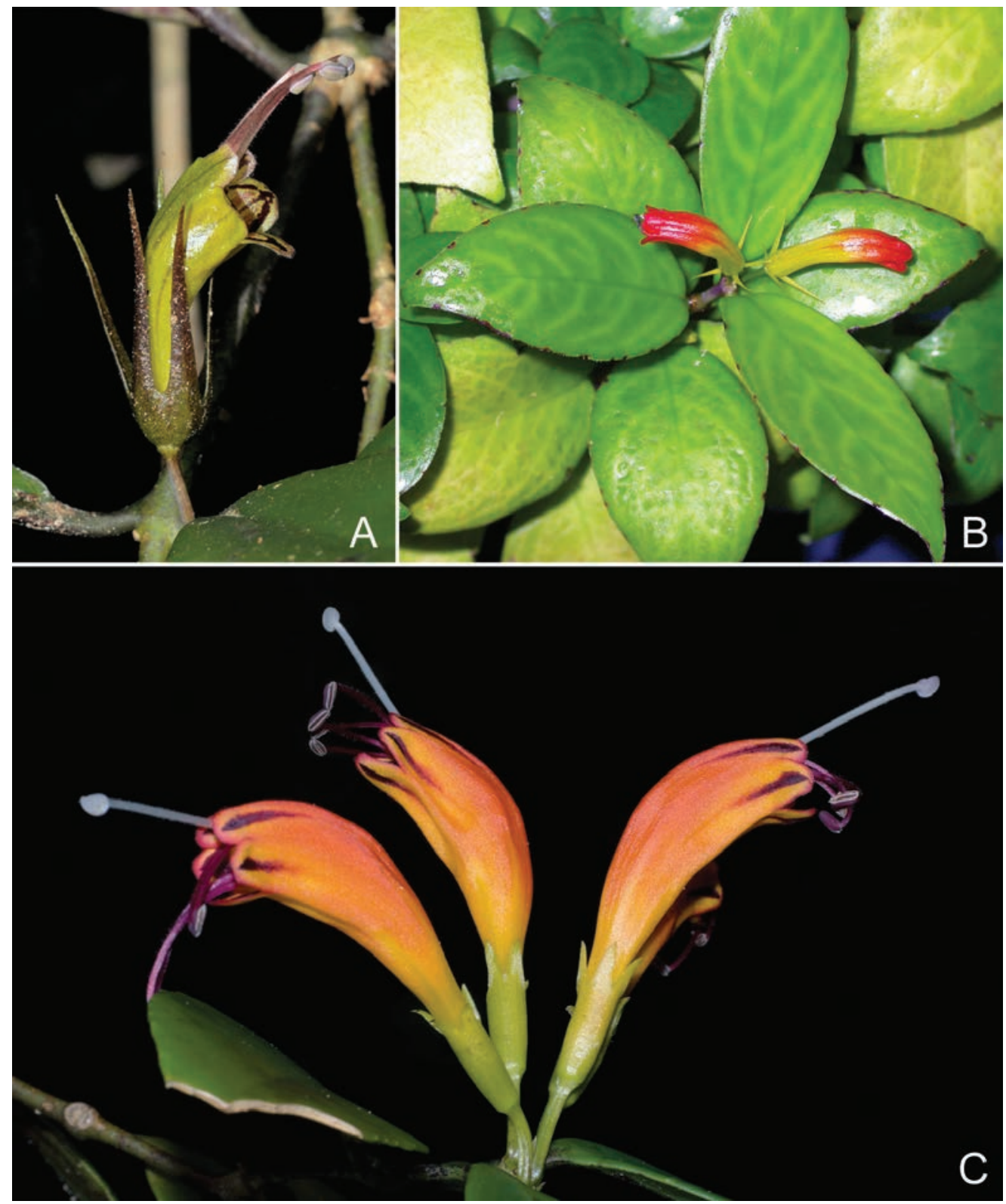

Fig. 1. A. Aeschynanthus albidus (Blume) Steud. B. Aeschynanthus fecundus P.Woods. C. Aeschynanthus fulgens Wall. ex R.Br. (Photos: A, Saw Leng Guan; B-C, David Middleton)

long; hilar appendages of many (30-40) filiform hairs, 14-19 mm long; appendages papillose.

Distribution. Peninsular Malaysia (Johor, Kelantan, Pahang, Perak, Selangor, 


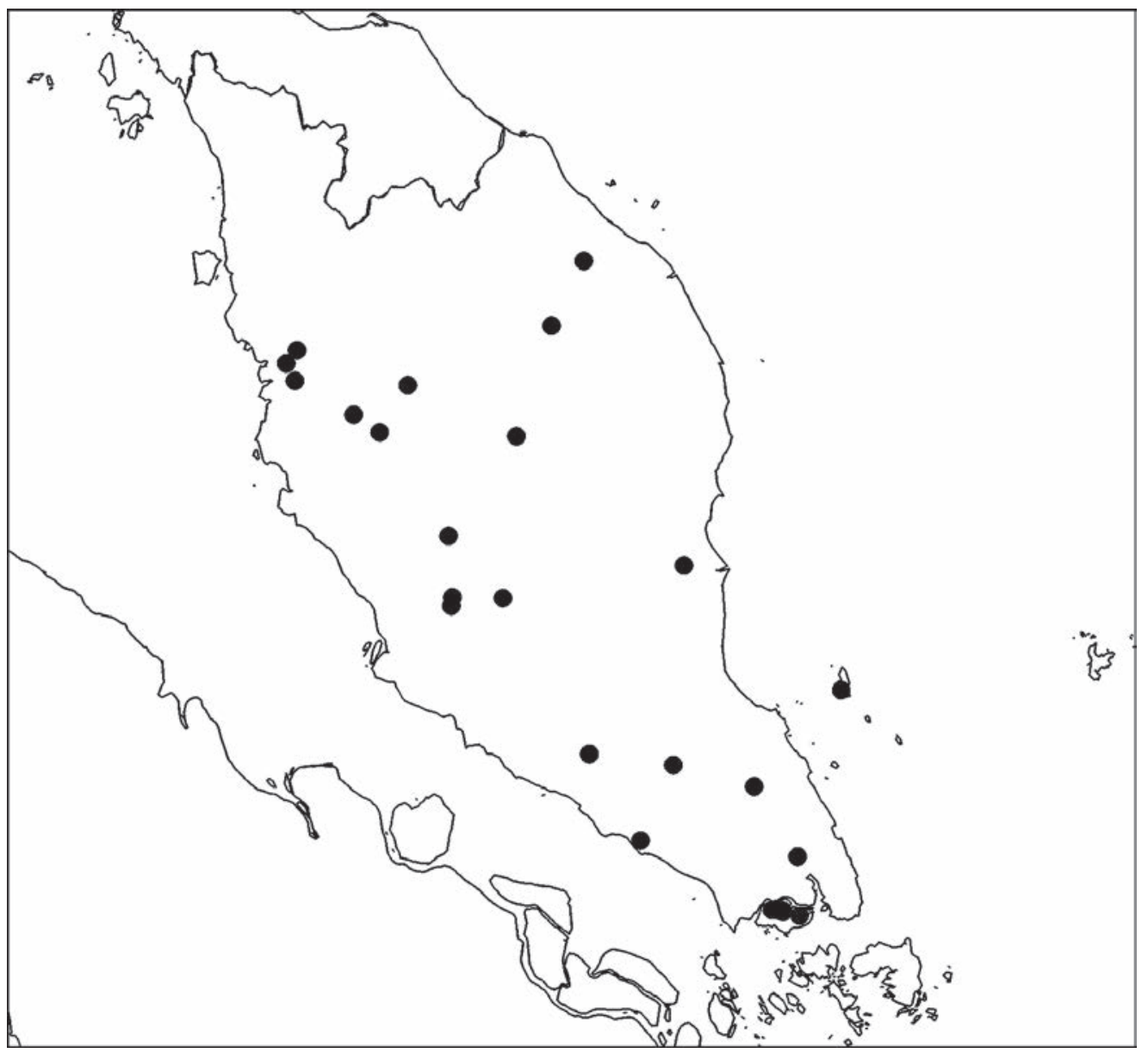

Fig. 2. Distribution of Aeschynanthus albidus (Blume) Steud. in Singapore and Peninsular Malaysia (•).

Terengganu), Singapore, Sumatra, Java, Borneo.

Habitat and ecology. In lowland mixed dipterocarp forest, sometimes by streams, or in lower montane forest at 20-1440 m altitude (to 1520 m on Mt Kinabalu).

Provisional IUCN conservation assessment. Least Concern (LC). This species is widespread and locally fairly common. In Peninsular Malaysia it has been collected over a fairly wide area in the last 20 years. In Singapore it is listed as nationally Critically Endangered by Chong et al. (2009). Although it was formerly more widespread it is now known in Singapore only in Nee Soon swamp forest.

Singaporean and Peninsular Malaysian specimens examined. PENINSULAR MALAYSIA: Johor: Gunong [Gunung] Ledang VJR, 19 Aug 1974, Kochummen, K.M. FRI16796 (K, KEP, SING); Lenggor Forest Reserve, Jong, K. 9017 (KLU); Batu Pahat, Nov 1891, Nongchi 8 
(SING); Kota Tinggi, 1 Oct 1929, Teruya, Z. 951 (SING); Bekok, Sungei Bekok, 7 Mar 1971, Heaslett, E.A. s.n. (SING); Kelantan: Kuala Aring, 7 Sep 1899, Yapp, R.H. 161 (CGE, K); Sungai Keteh, 6 Feb 1924, Md Nur \& Foxworthy, F.W. 11963 (SING); Gua Musang, Lojing FR, 860 m, 27 Apr 2011, Mohd Hairul, M.A., Siti Munirah, M.Y. \& Mohd Nazri, A. FRI72324 (KEP); Pahang: Jalan Bentong bt. 18, 13 Sep 1973, MK \& AR 1321 (L, UKMB); Fraser's Hill, 700 m, 10 Mar 1995, Chin, S.C. et al. 4533 (SING); Pulau Manis, Jul 1891, Ridley, H.N. 2151 (SING); Sabai Estate near Bentong, 26 Jan 1958, Shah, M. 155 (K, L, SING); Kuala Lipis, 22 Nov 1924, Burkill, H.M. \& Md Haniff 15750 (SING); Pulau Tioman, Kpg. Juara, 371 m, 25 Apr 2012, Saw, L.G. \& Mohd Hairul, M.A. FRI48326 (E, KEP); Perak: Gopeng, 150-240 m, Jun 1883, Kunstler, H. 4463 (NY, SING); Dipang, Mar 1885, Scortechini, B. 1815 (SING); Kurau, Wray, L. 4245 (SING); Taiping, Gunung Hijau, 1440 m, 19 Mar 2007, Julius, A. FRI53313 (E, KEP); Taiping, Bukit Larut, Jun 1893, Ridley, H.N. s.n. (SING); Kuala Kangsar, Bubu FR, Gunung Bubu, 12 Mar 2010, Julius, A., Coode, M.J.E. \& Angan, A. FRI57691 (KEP); Selangor: Genting Highlands, Ulu Gombak, 8 Apr 1921, Hume, H.L. 9596 (SING); ibidem, 16 Apr 1921, Hume, H.L. 9772 (SING); 17 mile Ulu Gombak, 26 Oct 1937, Md Nur SFN34254 (SING); Terengganu: Gunong [Gunung] Lawit, Old trail along Sungai Kamiah, 300-610 m, 1 Apr 1970, Davidson, C. 1292 (L).

SINGAPORE: s.l., 1894, Ridley, H.N. s.n. (SING); Seletar, 1894, Ridley, H.N. 6244 (SING); ibidem, 3 Sep 1889, Ridley, H.N. s.n. (SING); Bukit Mandai, Mar 1890, Ridley, H.N. s.n. (SING); Chan Chu Kang, Jul 1891, Ridley, H.N. s.n. (SING); ibidem, 1892, Ridley, H.N. s.n. (SING); Teban, 17 May 1891, Goodenough, J.S. s.n. (SING); Krangi [Kranji], 1894, Ridley, H.N. s.n. (SING); Nee Soon Swamp Forest, 22 Nov 2007, Ng, H.H. \& Lok, A.F.S.L. s.n. (SINU); Nee Soon Swamp Forest, 25 Mar 2015, Lua, H.K. \& Ibrahim, H. SING2015-084 (SING).

Notes. A very variable species, particularly in the size and relative dimensions of the calyx.

Burtt \& Woods (1975) gave the type as “Type: cult. Hort. Bogor (L?)”. However, in the protologue two collections are mentioned, one from "Tjoo" and one from "Salak". There is no mention of cultivated material from Bogor so the material designated as type by Burtt \& Woods (1975) cannot be considered original material. A Blume collection from Salak is, therefore, here chosen as lectotype.

This species has generally been called Aeschynanthus purpurascens or, less frequently, A. motleyi, in the literature from the Malay Peninsula. Aeschynanthus purpurascens is an illegitimate name as Aeschynanthus albidus and several other earlier combinations are included in synonymy. Aeschynanthus motley was described from Borneo but the Peninsular Malaysia, Borneo, Sumatra and Java material cannot be distinguished.

2. Aeschynanthus angustifolius (Blume) Steud., Nomencl. Bot. ed. 2 1: 32 (1840); A.DC., Prod. 9: 262 (1845); Miquel, Fl. Ned. Ind. 2: 716 (1858); C.B.Clarke in A.DC. \& C.DC., Monogr. Phan. 5(1): 38 (1883); Bakhuizen van den Brink, Blumea 6: 395 (1950); Backer \& Bakhuizen van den Brink, Fl. Java 2: 524 (1965); Woods, Kew Mag. 8(1): 22 (1991); Turner, Gard. Bull. Singapore 47(1): 243 (1997 ['1995’]). - Bignonia angustifolia Blume, Catalogus 82 (1823). - Trichosporum angustifolium (Blume) Nees, Flora 8: 144 (1825). - Lysionotus angustifolius (Blume) Blume, Bijdr. Fl. Ned. 
Ind. 765 (1826). - TYPE: Indonesia, Java, Jawa Barat, Bogor, Reinwardt, C.G.C. s.n. (lectotype L, designated here). (Fig. 3, 4)

Rheitrophyllum subverticillatum Hassk., Flora 25(2): beibl. 56 (1842). - TYPE: Java, Bogor Botanic Garden, 21 August 1840, Hasskarl s.n. (not traced). Although the type has not been traced the description of the leaves in the protologue is suitably diagnostic to be certain that it is this taxon.

Aeschynanthus tetraquetrus C.B.Clarke in A.DC. \& C.DC., Monogr. Phan. 5(1): 38 (1883). - Trichosporum tetraquetrum (C.B.Clarke) Kuntze, Revis. Gen. Pl. 478 (1891). - TYPE: Indonesia, Sumatra, Sumatera Barat, Gunung Singgalan, 1700 m, Beccari, O. s.n. (lectotype FI [FI013076], designated here; isolectotypes FI [FI013075], K).

Aeschynanthus stenophyllus Ridl., J. Asiat. Soc. Bengal 74 (2): 733 (1909); Ridley, Fl. Mal. Pen. 2: 498 (1923). - Trichosporum stenophyllum (Ridl.) Merr., Contr. Arnold Arbor. 8: 152 (1934). - TYPE: Peninsular Malaysia, Perak, Goping [Gopeng], Kinta, 150-240 m, August 1883, King's Collector 4738 (lectotype SING [SING0035634], designated by Woods (1991); isolectotype K).

Trichosporum ternifolium Merr., Contr. Arnold Arbor. 8: 153 (1934). - TYPE: Indonesia, Sumatra, in ravines between Baboeli and Paekas, 1200 m, 9 January 1932, Bangham, W.N. \& Bangham, C.M. 781 (holotype A [A00261780]; isotype NY [NY00313032]).

Epiphyte with pendulous or arching stems; stems glabrous. Leaves verticillate in whorls of 3-7, rarely opposite; petiole 1-6 mm long, glabrous; blade coriaceous or slightly fleshy, ovate or elliptic to linear, $1-8.6 \times 0.15-1.7 \mathrm{~cm}, 1.7-43$ times as long as wide (see note below), apex obtuse to acuminate, base cuneate, mid green above, paler beneath, not variegated, glabrous above and beneath, margin weakly and distantly dentate or entire, secondary veins obscure, tertiary veins obscure. Flowers solitary in the axils of leaves, bracts minute; pedicels 9.5-10 mm long, glabrous. Calyx of separate lobes free to base, green, glabrous except with very few hairs at tips of lobes; lobes narrowly triangular, 1.5-4.2 × 0.7-0.9 mm, apex acute, glabrous. Corolla 18-25 mm long, tube fairly narrow, slightly wider at base, fairly straight, curved at apex, externally yellowish to yellowish green to green, margins of lobes reddish brown, internally yellowish green, reddish brown on margins of lobes and speckled on inner lobes; upper lobes oblong, not spreading or reflexed, 2.2-3.8 $\times 2.2-3.2 \mathrm{~mm}$, sinus 2.6$2.8 \mathrm{~mm}$ deep, apex rounded; lateral lobes oblong, not spreading or reflexed, 3.5-4.3 $\times$ 3-3.2 mm, apex rounded; lower lobe oblong, slightly spreading, 3.5-6 × 2.5-4 mm, apex rounded; tube and outside of lobes minutely glandular puberulent, lobes ciliate, inside of lobes and tube with scattered short glandular hairs. Stamens shortly exserted, fused in 2 pairs; filaments glandular pubescent; anterior filaments inserted at 11-15 $\mathrm{mm}$ from corolla base which is $61-65 \%$ of corolla length, filaments $10-12 \mathrm{~mm}$ long, anthers $1.2-1.5 \times 0.8-0.9 \mathrm{~mm}$; posterior filaments inserted at $12-16 \mathrm{~mm}$ from corolla 

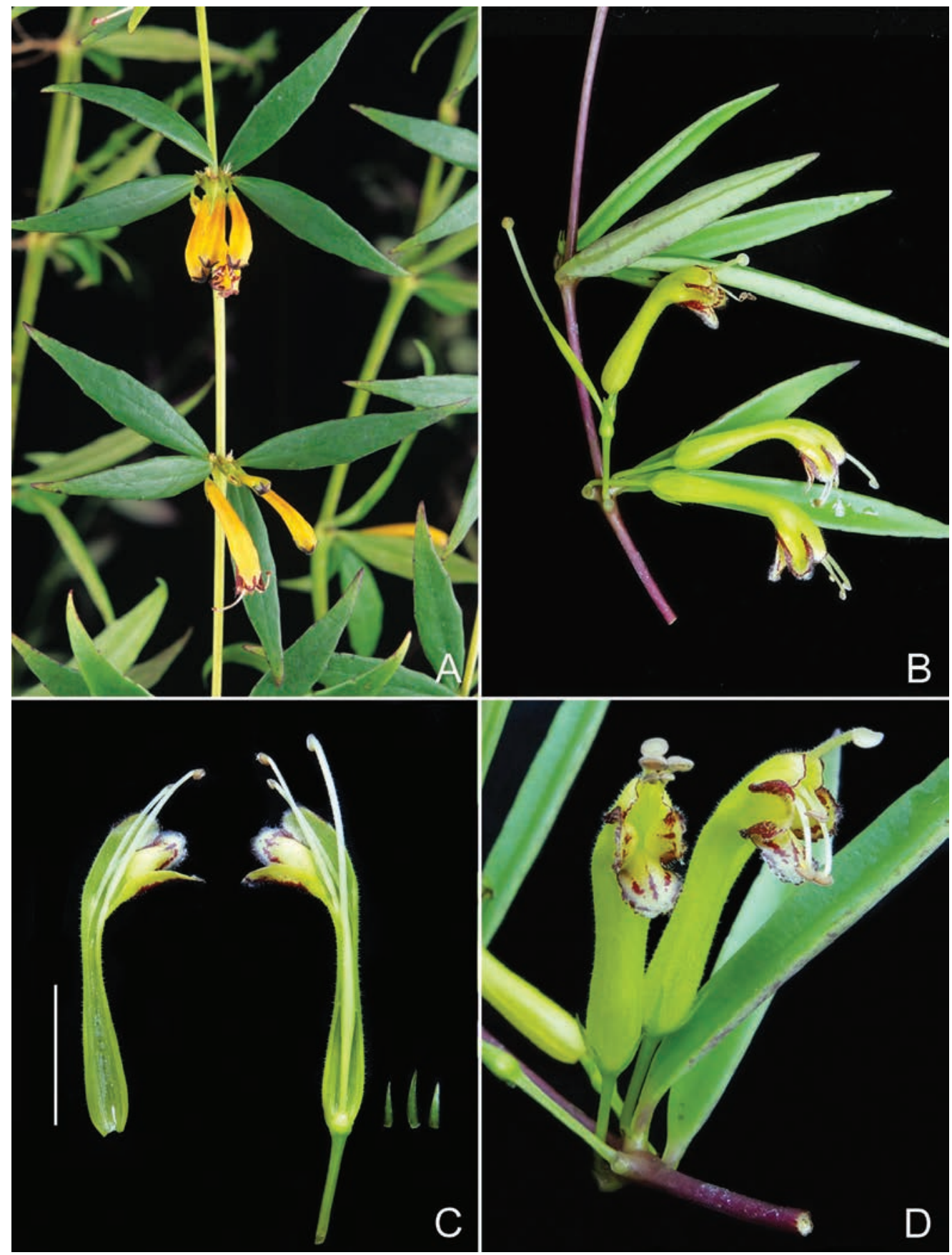

Fig. 3. Aeschynanthus angustifolius (Blume) Steud. A. Habit with yellow-flowered form. B. Habit with green-flowered form. C. Flower dissection with three calyx lobes removed. D. Flowers from the front showing stamens in two pairs and developing style and stigma in the flower on the left and a more developed style and the stamens beginning to wither and reflex in the flower on the right. (Photos: A, David Middleton; B-D, Jana Leong-Škorničková) 


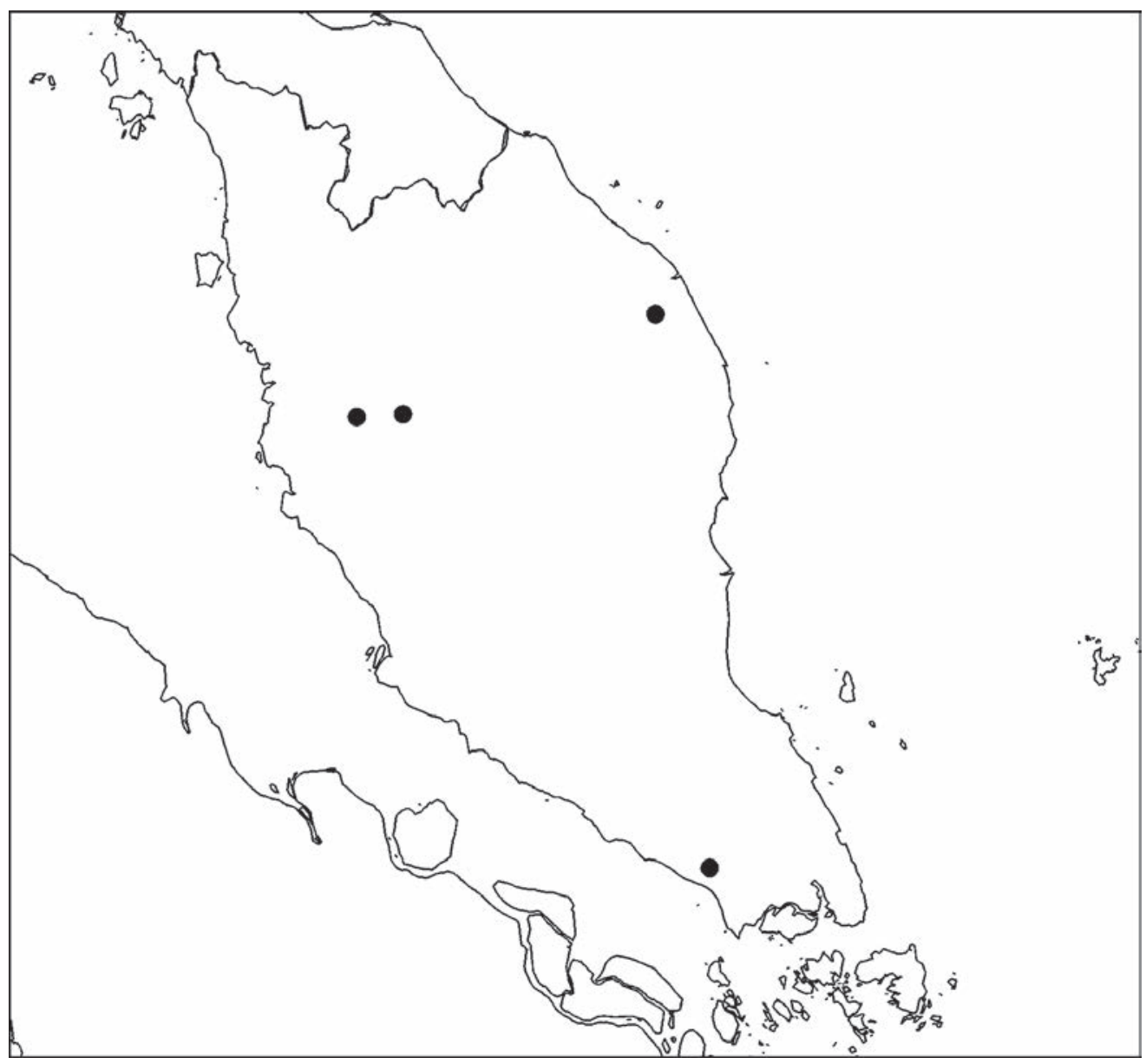

Fig. 4. Distribution of Aeschynanthus angustifolius (Blume) Steud. in Peninsular Malaysia (•).

base which is $67-73 \%$ of corolla length, filaments $8.5-9 \mathrm{~mm}$ long, anthers $0.8-1.2$ $\times$ 0.6-0.7 mm; staminode c. $0.5 \mathrm{~mm}$ long. Disk 1.2-1.5 mm high, margin 5-crenate. Pistil 28-30 mm long, pale green; stipe 5-6 mm long, with sparse sessile glands; ovary 8-9 mm long, with sparse sessile glands; style c. $15 \mathrm{~mm}$ long, with short glandular hairs. Capsule 13-30 cm long, 2.4-3.3 mm wide. Seed grain 1.6-1.9 × 0.3 mm, bubble cells absent; apical appendage a filiform hair 44-50 mm long; hilar appendage a single filiform hair, 28-30 $\mathrm{mm}$ long.

Distribution. Peninsular Malaysia (Johor, Pahang, Terengganu), Sumatra, Java, Borneo.

Habitat and ecology. Primary or secondary lowland to lower montane forest. In Peninsular Malaysia recorded from 150-1390 m altitude and in Sumatra recorded up to $1700 \mathrm{~m}$. 
Provisional IUCN conservation assessment. Data Deficient (DD). The EOO and AOO for this species using the existing collections would suggest an assessment of Least Concern. However, all of the collections I have seen of this species collected in the last 20 years have been made only in Borneo over a range that would suggest Near Threatened. I have seen no collections from Peninsular Malaysia made since 1930 and its current status there is uncertain. It has never been collected in Singapore.

Additional Peninsular Malaysian specimens examined. PENINSULAR MALAYSIA: Johor: Sedenak, Nov 1909, Md Nur s.n. (SING); ibidem, 1909, Ridley, H.N. s.n. (SING); Pahang: Cameron Highlands, 1390 m, 1 Apr 1930, Henderson, M.R. s.n. (NY); Terengganu: Kuala Berang, 14 May 1925, Holttum, R.E. 15312 (K, SING).

Notes. Leaf characters are extremely variable within this species even though most material does show linear leaves $>7$ times as long as wide. Wide variation occurs even within individuals as was previously commented upon by Woods (1991). For example, Poulsen et al. 1566 (KEP) from Long Maga, Ulu Padas, Sabah, has leaves from 1.7-30 times as long as wide on a single branch. In this case there appear to have been two distinct phases of growth with older, shorter and wider leaves on the older part of the branch and then a new flush of growth with linear leaves on the younger part of the branch.

This species has been described as several different species from the different parts of its range due to differences in corolla colour and leaf shape but there are no clear discontinuities in these characters across its range.

3. Aeschynanthus dischidioides (Ridl.) D.J.Middleton, Edinburgh J. Bot. 64: 425 (2007). - Micraeschynanthus dischidioides Ridl., Fl. Malay Penin. 5: 325 (1925). - TYPE: Peninsular Malaysia, Pahang, Gunung Tahan, 1670 m, Ridley, H.N. 16122 (lectotype K, designated by Middleton (2007); isolectotype SING [SING0089714]). (Fig. 5, 6)

Aeschynanthus myrmecophilus P.Woods, Notes Roy. Bot. Gard. Edinburgh 33: 483 (1975); Turner, Gard. Bull. Singapore 47(1): 243 (1997 ['1995’]). - TYPE: Peninsular Malaysia, Pahang, Cameron Highlands, Robinson’s Falls, 1500 m, 16 April 1968, Woods, P.J.B. 616 (holotype E [E00062778]).

Aeschynanthus hildebrandii auct. non Hemsl. ex Hook.f.: Ridley, J. Linn. Soc. Bot. 32: 502 (1896); Ridley, J. Straits Branch Roy. Asiat. Soc. 44: 15 (1905); Ridley, J. Asiat. Soc. Bengal 74(2): 734 (1909); Ridley, Fl. Mal. Pen. 2: 499 (1923).

Epiphyte, often rooted in ants nests, with stems hanging; stems sparsely puberulent or glabrous. Leaves opposite (although sometimes appearing whorled due to very short internodes); petiole 1-3 mm long, purple, glabrous; blade thickly coriaceous, green edged with purple above, not variegated, green to reddish purple beneath, ovate 
or orbicular, 0.7-2.4 cm long, 0.6-2.6 cm wide, 1-2.4 times as long as wide, apex rounded to acuminate, base rounded or subcordate, glabrous above and beneath, not punctate beneath, margin dentate or entire, undulate or not, secondary veins obscure, tertiary veins obscure. Inflorescence axillary or terminal, 1-6-flowered; pedicels 8.5$16 \mathrm{~mm}$ long, green or purple, with sparse and minute hairs, smaller ones more or less papillae, hairs (papillae) green or red in life. Calyx of separate lobes free to base, green, with sparse and minute hairs; lobes narrowly ovate or narrowly triangular, erect, 2-6.5 × 0.6-1.6 mm, apex acute. Corolla 11-25 mm long, tube broad for all of length, slightly to strongly curved, outside of tube yellow to yellow-orange for most of length and then abruptly turning to dark orange or red on top of tube and lobes, sometimes only on lobes, inside of tube yellow, lobes dark red to orange-red at margin and yellow at base with dark bands on lower 3 lobes, outside sparsely puberulent, denser on ciliate lobes, inside with a ring of robust multicellular hairs in lower half; upper lobes oblong, not spreading or reflexed, conspicuously shorter than neighbouring lateral lobes, 2.6-4 × 2.5-4.8 mm, sinus 3.2-4.2 mm deep, apex rounded; lateral lobes ovate or oblong, not spreading or reflexed, 3.6-6 $\times 2.9-5.5 \mathrm{~mm}$, apex rounded, truncate or retuse; lower lobe oblong, not spreading or reflexed, 4.9-6 $\times 3-5.8 \mathrm{~mm}$, apex rounded to truncate. Stamens clearly exserted, fused in 2 pairs; filaments various shades of green to red, posterior pair glandular hairy at apex and papillose at base, anterior pair only papillose, anthers grey; anterior filaments inserted at 8-11 $\mathrm{mm}$ from corolla base which is $44-62 \%$ of corolla length, filaments $12-16 \mathrm{~mm}$ long, anthers $1.4-2 \times 0.6-1.2$ $\mathrm{mm}$; posterior filaments inserted at $9.5-11.5 \mathrm{~mm}$ from corolla base which is $48-69 \%$ of corolla length, filaments $10.5-13 \mathrm{~mm}$ long, anthers $1.1-1.6 \times 0.5-1 \mathrm{~mm}$; staminode 0.7-1 mm long. Disk 1.2-1.5 mm high, 5-dentate. Pistil 21-24 mm long; stipe 2.5-3.5 mm long, glabrous or with sparse sessile glands; ovary green, 6-6.5 mm long, with sessile glands, these sometimes sparse; style 13-14 mm long, glandular pubescent. Capsule 8-24 cm long, 2.7-3 mm wide. Seed grain 1.2-1.6 × 0.3-0.4 mm, warty, bubble cells absent; apical appendage a filiform hair, 10-50 mm long; hilar appendage of 3-6 filiform hairs, $17-30 \mathrm{~mm}$ long.

Distribution. Endemic in Peninsular Malaysia (Pahang, Perak, Selangor).

Habitat and ecology. In lower montane forest at 1100-2000 m altitude, often growing in ant gardens associated with Lecanopteris or Dischidia.

Provisional IUCN conservation assessment. Vulnerable (VU B1ab(iii)). The known EOO is around 15,000 $\mathrm{km}^{2}$ (but see note below) and the locations where it occurs are disturbed by farming, plantations and tourism.

Additional Peninsular Malaysian specimens examined. PENINSULAR MALAYSIA: Pahang: Cameron Highlands, 1390 m, 1 Apr 1930, Henderson, M.R. s.n. (NY, SING); ibidem, 9 Mar 1947, Smith, J.W. 63690 (KEP, SING); ibidem, 14 Oct 1929, Symington, C.F. 20938 (KEP); ibidem, 1976, Anthony, S. SA230 (KEP); Cameron Highlands, Along route no. 7 to G. Beremban, 1500 m, 3 Mar 1994, Perumal, B., Gan, C.L., Shahril, K.Z., Angan, A. \& Bedul FRI41631 (KEP, KLU); Cameron Highlands, Gedung FR, Near Sg. Ichat tea plantation, 1365 


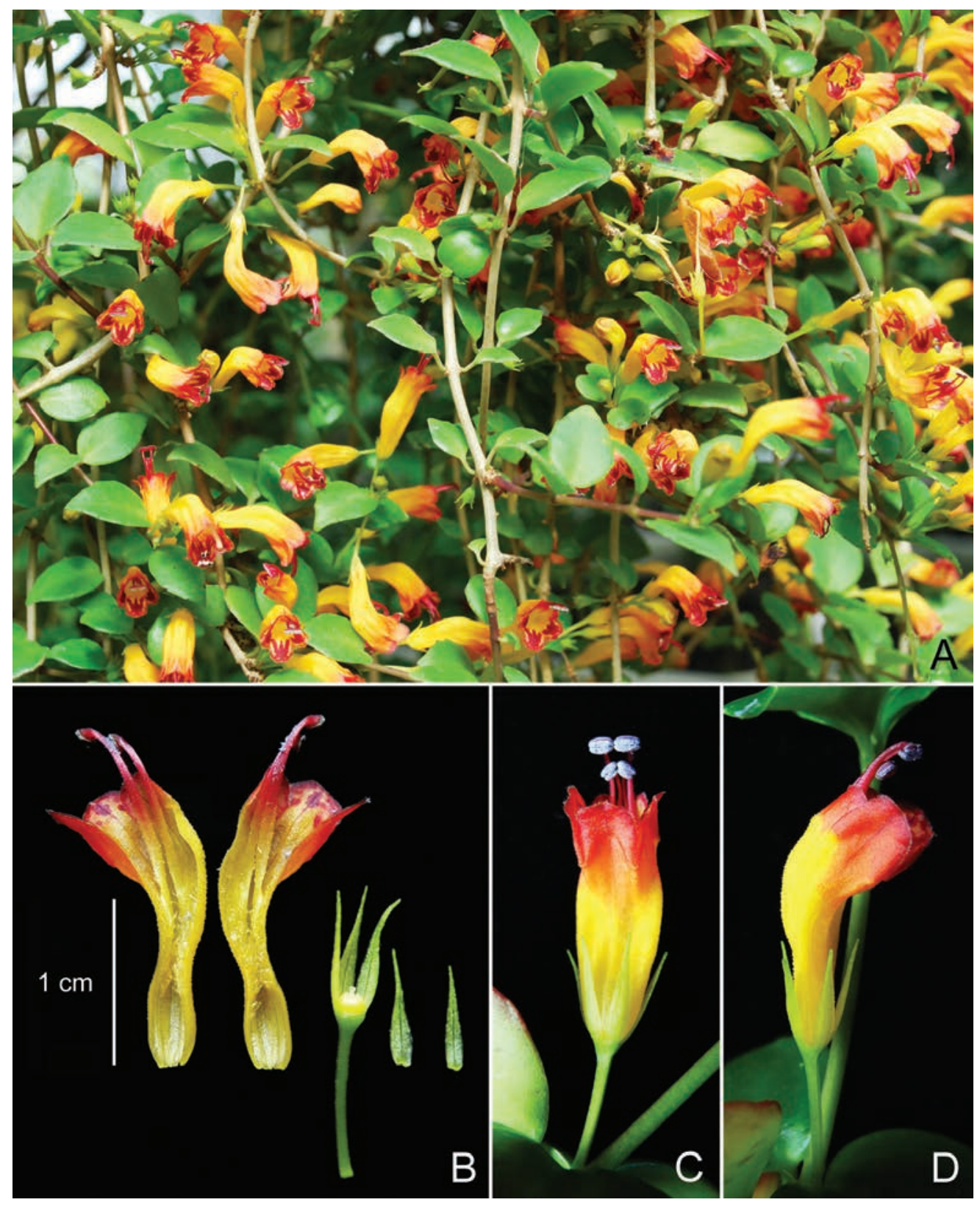

Fig. 5. Aeschynanthus dischidioides (Ridl.) D.J.Middleton. A. Habit. B. Flower dissection showing multicellular hairs in corolla tube and undeveloped style after two calyx lobes removed. C. Flower from below with stamens fused in two pairs. D. flower, side view. (Photos: A, David Middleton; B-D, Jana Leong-Škorničková) 


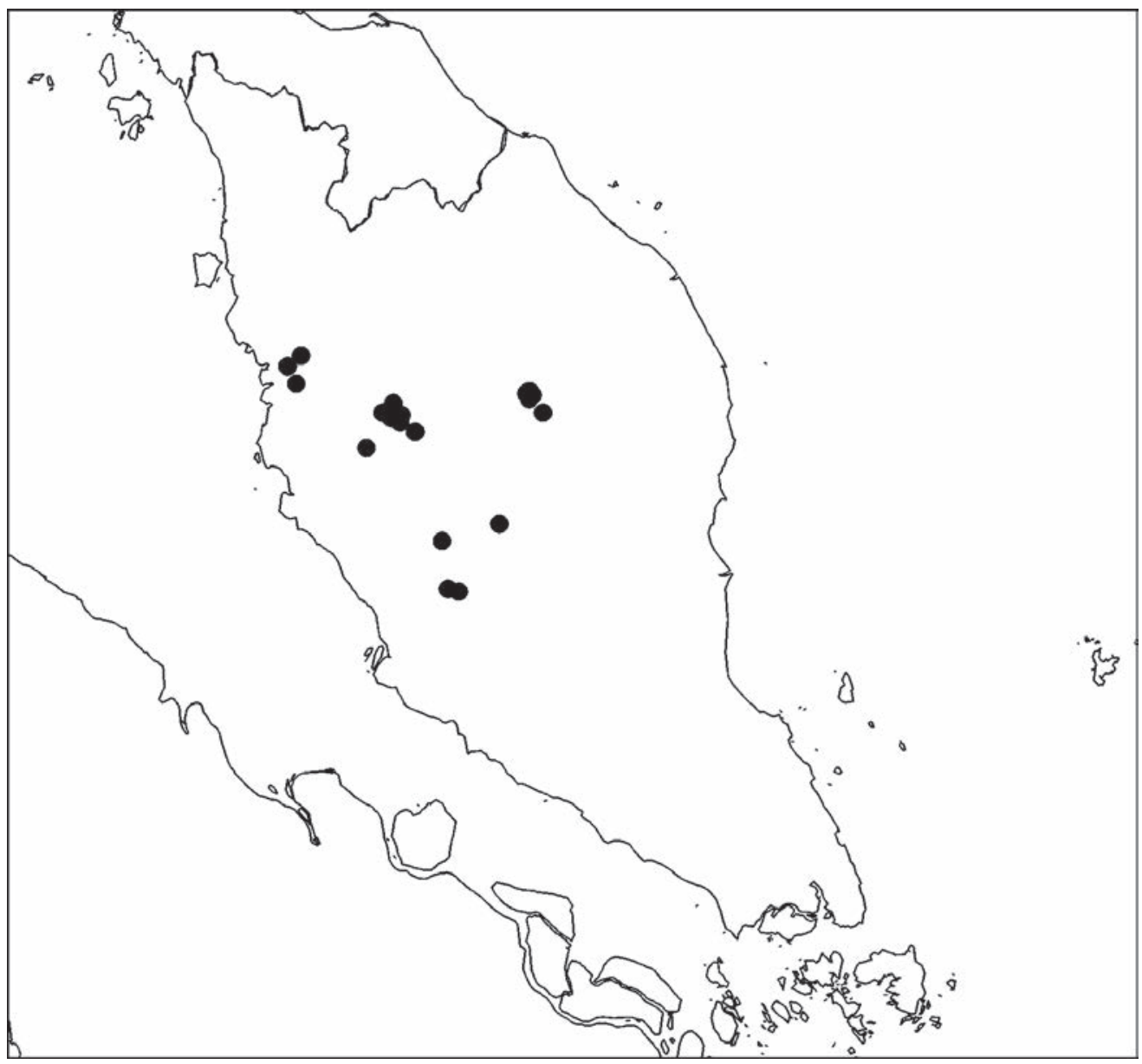

Fig. 6. Distribution of Aeschynanthus dischidioides (Ridl.) D.J.Middleton in Peninsular Malaysia (•).

m, 19 Feb 2008, Syahida-Emiza \& Chew, M.Y. FRI57277 (KEP); Cameron Highlands, G. Jasar, 9 Aug 2008, Rosdi, M. \& Phoon, S.N. et al. FRI59872 (KEP); Cameron Highlands, Gunung Batu Berinchang, Kpg. Melayu Brinchang, 22 Feb 2012, Imin, K. \& Syahida-Emiza FRI76065 (KEP); Cameron Highlands, Gunung Batu Berinchang, 2000 m, 20 Jun 1975, Van Balgooy, M.M.J. 2658 (E, L); Cameron Highlands, Boh Tea Estate, 29 Apr 1937, Md Nur SFN32980 (SING); ibidem, 28 Jul 1991, Kiew, R. RK3241 (SING); Cameron Highlands, Tanah Rata, 18 Aug 1986, Weber, A. s.n. (KEP); ibidem, 1440 m, 9 Sep 1956, Burkill, H.M. HMB868 (SING); Cameron Highlands, Tanah Rata, Parit Waterfall, 1457 m, 26 Sep 2012, Imin, K. et al. FRI77536 (E, KEP, SING); Cameron Highlands, Tanah Rata, Jln Tenkolok, 1489 m, 23 Feb 2012, Imin, K. \& Syahida-Emiza FRI76073 (KEP); Cameron Highlands, Robinson's Falls, 5 Apr 2006, Kiew, R. RK5307 (SING); Cameron Highlands, en route from Robinson Waterfall to Boh tea plantation, 600-1100 m, 29 Aug 1990, Okada, H., Darnaedi, D., Akiyama, H., Kawahara, T. \& Watano, Y. 1050 (TI); Taman Negara, 1100-1530 m, 18 Aug 1990, Darnaedi, D., Akiyama, H., Kawahara, T. \& Khairuddin, K. 566 (TI); Taman Negara, Kem Bonsai, From Kem Bonsai to Kem Belumut, 1985 m, 8 May 2008, Yao, T.L. FRI65302 (E, KEP); 
Taman Negara, Gunung Tahan, 1820m, 1 Sep 1928, Holttum, R.E. s.n. (SING); ibidem, 7 Jul 1905, Wray, L. \& Robinson, H.C. 5483 (BM, K, SING); ibidem, Jul 1911, Ridley, H.N. s.n. (SING); Gunung Tahan, Foot of Gunung Gedong, 1520 m, 3 Sep 1928, Holttum, R.E. 20772 (SING); Gunung Tahan, Skeats Hill, Jul 1911, Ridley, H.N. s.n. (SING); Fraser's Hill, Pine tree hill, 1450 m, 19 Apr 1955, Purseglove, J.W. P4222 (K, SING); ibidem, 1450 m, 3 Sep 1966, Burkill, H.M. HMB4221 (SING); Padang Camp, 1670m, 14 Sep 1937, Corner, E.J.H. s.n. (L); Kluang Tabang, 1900, Barnes, E. s.n. (SING); Perak: Gunong [Gunung] Jasar, 17 Apr 1968, Woods, P.J.B. 634 (E); Taiping, 1210 m, 14 Feb 1917, Md Haniff \& Md Nur 2469 (K, SING); ibidem, 1240 m, 14 Feb 1917, Md Haniff \& Md Nur 2347 (SING); Taiping, Bukit Larut, Jun 1893, Ridley, H.N. \& Curtis, C. 7365 (SING); Kuala Kangsar, Bubu FR, Gunung Bubu, 1675 m, 20 Dec 2006, Kamarul Hisham, M., Kueh, H.L., Lim, C.L. \& Yao, T.L. FRI52094 (KEP); Selangor: Ulu Kali, 1600 m, 2 Mar 1996, Van Balgooy, M.M.J. 7149 (L).

Notes. This is currently the only species of Aeschynanthus considered to be endemic to Peninsular Malaysia. However, considering that most of its distribution closely mirrors that of Aeschynanthus rhododendron, suggesting similar habitat requirements, it may also occur in the far south of Thailand and in Sumatra where A. rhododendron has been collected.

The combination in Aeschynanthus was published by Middleton (2007) when the monotypic Micraeschynanthus dischidiodes was synonymised with Aeschynanthus myrmecophilus. Middleton (2007) concluded that the type material of Micraeschynanthus dischidiodes has only immature and aberrant flowers but that in all other characters it matches Aeschynanthus myrmecophilus, thereby requiring synonymisation and a new combination in Aeschynanthus.

4. Aeschynanthus fecundus P.Woods, Notes Roy. Bot. Gard. Edinburgh 33: 482 (1975); Turner, Gard. Bull. Singapore 47: 243 (1997 ['1995’]); Burtt, Thai Forest Bull., Bot. 29: 83 (2001); Smitinand, Thai Pl. Names ed. 2, 14 (2001); Middleton, Edinburgh J. Bot. 64: 379 (2007). - Aeschynanthus parviflorus Ridl., J. Fed. Malay States Mus. 4: 48 (1909), nom. illeg. - Aeschynanthus breviflorus Ridl., Fl. Mal. Pen. 2: 497 (1923), nom. illeg. - TYPE: Peninsular Malaysia, Pahang, Telom, November 1900, Ridley, H.N. 13599 (holotype SING [SING0089822]; isotypes BM [BM000537160], K [K000190151]). (Fig. 1, 7)

Epiphyte with erect, arching or pendulous stems; stems green, flushed purplish or reddish brown, glabrous. Leaves opposite; petiole green, 2-4 mm long, glabrous; blade slightly fleshy, green with paler green or yellow variegation above, pale green beneath, elliptic, 2.1-8.6 × 0.5-3.7 cm, 1.7-5.3 times as long as wide, apex acuminate, base rounded to cuneate, glabrous above and beneath, not punctate beneath, margin with few minute red-tipped teeth but appearing more or less entire, secondary veins obscure, tertiary venation obscure. Inflorescences axillary, 1-2-flowered, peduncle absent; pedicels green, 4-7 mm long, sparsely minutely papillose or glabrous. Calyx of separate lobes free to base, yellow-green or green, tips red, glabrous or with sparse sessile glands; lobes linear to narrowly ovate, slightly spreading or erect, 2.3-13.5 


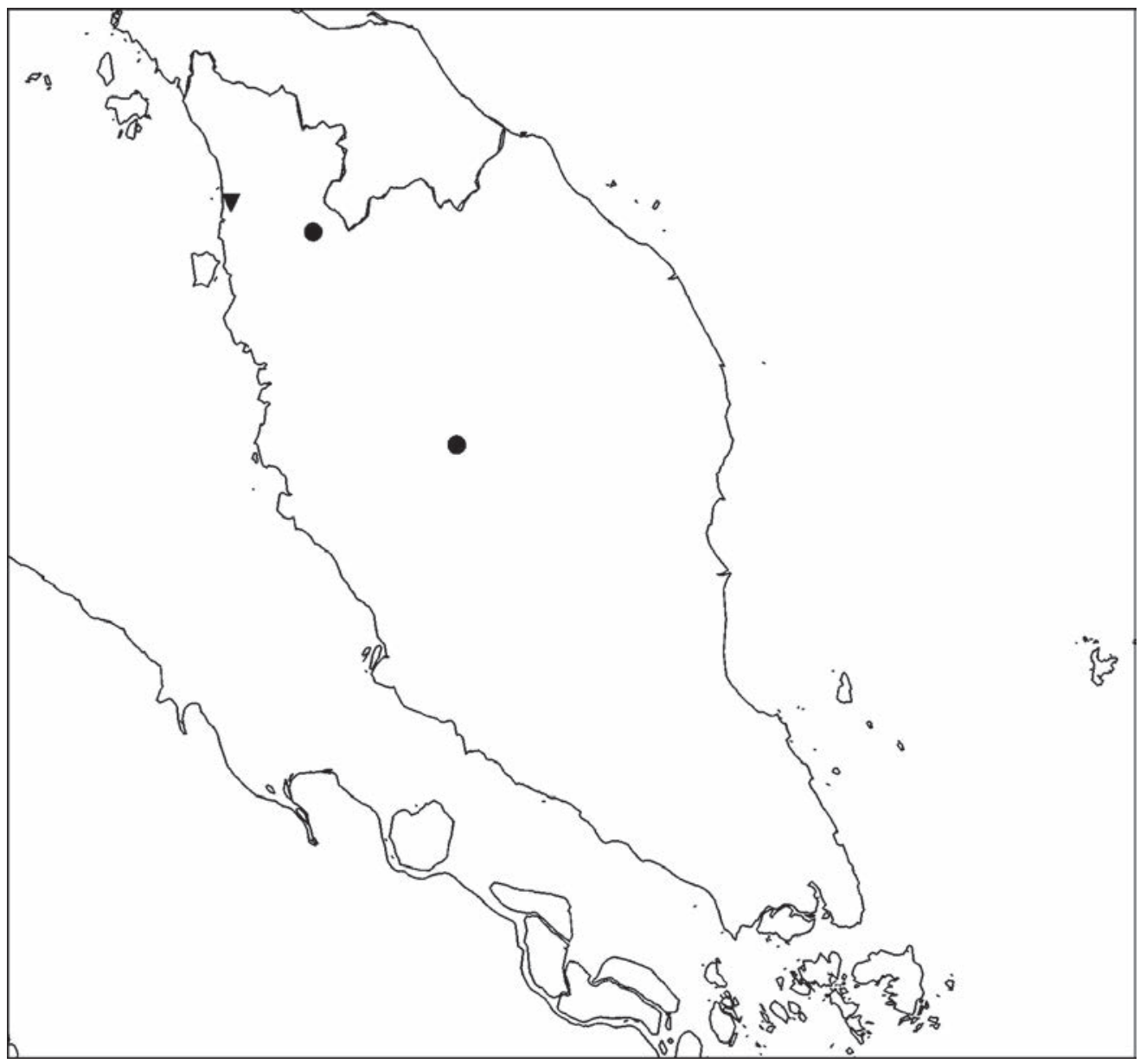

Fig. 7. Distribution of Aeschynanthus fecundus P.Woods $(\bullet)$ and Aeschynanthus fulgens Wall. ex R.Br. ( $\boldsymbol{\nabla})$ in Peninsular Malaysia

× 0.6-1.5 mm, apex acute or acuminate. Corolla 14.5-19 mm long, externally tube yellowish green or yellow in lower two-thirds, brownish red higher, lobes dark red or brownish red, internally tube light yellowish, lobes pale brownish red, tube fairly broad at base; upper lobes oblong or elliptic, not spreading or reflexed, 1.1-2.3 $\times 1.2-2.3 \mathrm{~mm}$, sinus 1.4-2.6 mm deep, apex rounded; lateral lobes ovate, not spreading or reflexed, 1.1-2.5 × 1.6-2.9 mm, apex rounded; lower lobe elliptic, not spreading or reflexed, $1.3-2.3 \times 1-2.6 \mathrm{~mm}$, apex rounded; glabrous or with few sessile glands around top outside, with irregular tufts of multicellular hairs near base and glandular papillose on ventral surface near throat inside. Stamens not exserted, fused in 2 pairs, filaments yellow or greenish yellow, with glandular hairs, anthers yellow to grey, pollen cream; anterior filaments inserted at $7-8.6 \mathrm{~mm}$ from corolla base which is $45-48 \%$ of corolla length, filaments 7-9 mm long, anthers $1-1.3 \times 0.6 \mathrm{~mm}$; posterior filaments inserted at $8-12 \mathrm{~mm}$ from corolla base which is $55-63 \%$ of corolla length, filaments c. $6 \mathrm{~mm}$ long, 
anthers $0.7-0.9 \times 0.5-0.6 \mathrm{~mm}$; staminode 0.8-2 mm long. Disk $1 \mathrm{~mm}$ high, a simple annular ring. Pistil 17-19 mm long; stipe c. $1.5 \mathrm{~mm}$ long, with few sessile glands; ovary green, 4.5-5.5 mm long, with sessile glands; style green or yellowish green, 11-12 mm long, glandular pubescent; stigma pink or purple. Capsule 3.6-12 cm long, 1.9-2.8 mm wide. Seed grain 2-2.5 $\times 0.2-0.3 \mathrm{~mm}$, only weakly warty, bubble cells absent; apical appendage a filiform hair, 19-28 mm long; hilar appendage of 11-18 filiform hairs, 12-17 $\mathrm{mm}$ long, appendages papillose.

Distribution. Western and southern Thailand, Peninsular Malaysia (Kedah).

Habitat and ecology. In lowland and hill dipterocarp forest, sometimes on bamboo. Altitude information is not available for the species in Peninsular Malaysia but in Thailand it occurs up to 700 m altitude.

Provisional IUCN conservation assessment. Least Concern (LC). Even though this species has been collected only once in recent years in Peninsular Malaysia, and only twice ever, in Thailand it has a wide distribution and has been collected fairly often.

Additional Peninsular Malaysian specimens examined. PENINSULAR MALAYSIA: Kedah: Baling, Gunung Inas Forest Reserve, Bukit Palong, 2 Nov 2007, Imin, K. et al. FRI58593 (K, KEP, SING).

Notes. This species is difficult to tell apart from Aeschynanthus longicaulis when in fruit. In Thailand this species was once found in fruit with the seeds already germinating before the seeds had dispersed. It is not known if this was an aberration or common occurrence.

5. Aeschynanthus fulgens Wall. ex R.Br., Cyrtandreae 115 (1839); Steudel, Nomencl. Bot. ed. 2, 1: 32 (1840); Brown in Bennett, Pl. Jav. Rar. 115 (1840); A.DC., Prod. 9: 261 (1845); C.B.Clarke in A.DC. \& C.DC., Monogr. Phan. 5(1): 21 (1883); Clarke in Hooker, Fl. Brit. Ind. 4: 338 (1884); Burtt, Thai Forest Bull., Bot. 29: 83 (2001); Smitinand, Thai Pl. Names ed. 2, 14 (2001); Kress et al., Checkl. Myanmar 261 (2003); Pooma, Threatened Pl. Thailand 70 (2005); Middleton, Edinburgh J. Bot. 64: 381 (2007); Middleton, Edinburgh J. Bot. 66: 414 (2009). - Trichosporum fulgens (Wall. ex R.Br.) Kuntze, Revis. Gen. Pl. 477 (1891). - TYPE: Burma, Tenasserim Division, Tavoy, Gomez, W. in Wallich 797 (lectotype K-W, designated by Middleton (2007); isolectotypes BM [BM000883874], CGE, E [E00259870, E00259871], G [G00370743], G-DC, K [K000831871, K000831872]). (Fig. 1, 7)

Aeschynanthus evrardii Pellegr., Bull. Soc. Bot. France 72: 824 (1926 [1925]); Lecomte, Fl. Indo-Chine 4: 499 (1930); Pham Hoang Ho, Cayco Vietnam ed. 3, 3 (1): 4 (1993); Newman et al., Checkl. Vasc. Pl. Lao PDR 146 (2007). - TYPE: Vietnam, Lam Dong, Ang Kroet, 26 October 1920, Evrard, F. 358 (lectotype P [P00492372], designated by Middleton (2007); isolectotypes P [P00606317, P00606318]). 
Aeschynanthus stenosiphonius W.T.Wang, Bull. Bot. Res., Harbin 3 (4): 49 (1983); Burtt, Thai Forest Bull., Bot. 29: 84 (2001); Smitinand, Thai Pl. Names ed. 2, 15 (2001). - TYPE: Laos, Vieng Pa Pow Tha Kaw, 2 November 1921, Hayata, B. s.n. (holotype TI; isotype TI).

Hoya pseudolanceolata Costantin, Fl. Indo-Chine 4: 132 (1912). - TYPE: Laos, Sayabouri, Pak Lay, Spire 1490 (lectotype P [P00639825], designated here).

Aeschynanthus macranthus auct. non (Merr.) Pellegr.: Pellegrin, Fl. Indo-Chine 4: 498 (1930) pro parte; Barnett, Fl. Siam. 3 (3): 201 (1962); Burtt, Thai Forest Bull., Bot. 29: 84 (2001).

Epiphyte with stems arching and pendulous or upright, green or dull olive-green, sometimes with purple mottling, glabrous. Leaves opposite; petiole 3-20 mm long, glabrous; blade coriaceous to fleshy, elliptic, oblong or ovate, mid to dark green above, paler green beneath, not variegated, 3.3-17 × 1-5.2 cm, 1.7-7.5 times as long as wide, apex acuminate, base cuneate to rounded, glabrous above and beneath, margin entire, 4-8 pairs of secondary veins, obscure to clearly visible, tertiary venation obscure. Inflorescences subterminal or axillary, 3-16-flowered, peduncle absent; bracts deep maroon, ovate or linear, 2.1-11 mm long; pedicels 3.5-24 mm long, green, glandular puberulent or glabrous. Calyx with a tube at base, lobes free, tube very variable in width from narrow and clasping corolla tube to quite wide and not clasping corolla, yellowish, greenish or brownish, sometimes with reddish lobes, glabrous except for ciliate lobes, or few hairs only on very tips of lobes, to glandular pubescent all over, total length (7-)10.7-26 mm long; tube (2.4-)6-18.5 mm long which is (34-)50-94\% of total length, 3.5-14 mm wide at top of tube; lobes triangular, slightly spreading or erect, $1-5(-11.5) \times(1.2-) 1.6-6.5 \mathrm{~mm}$, apex acute, sometimes ultimately with a rounded tip, to acuminate. Corolla (41-)49-73 mm long, tube narrow at base, externally bright red to darker red or orange red, sometimes yellowish at base, usually with darker lines on tube, red or orange-red with a black central line on lobes, sometimes yellowish at very base, internally yellowish to pale red in tube, lobes orange-red or red with a dark red or black central line or arrow and pale orange to cream at base; upper lobes slightly falcate, mostly not reflexed or spreading, sometimes slightly spreading but not fully reflexed, 3.2-7.5 $\times 3.3-7 \mathrm{~mm}$, sinus $3.5-10.5 \mathrm{~mm}$ deep, apex obtuse or rounded; lateral lobes deltoid, not spreading or reflexed, $2.8-8 \times 5.2-9.5 \mathrm{~mm}$, apex rounded or obtuse; lower lobe oblong or elliptic, reflexed, 5.1-14 $\times 3-6 \mathrm{~mm}$, apex obtuse or rounded; sparsely to densely glandular puberulent outside or only at top of tube and on lobes or only ciliate on lobes, minutely and sparsely to very sparsely glandular puberulent internally, this sometimes denser towards the upper half, sessile glands present at top of tube. Stamens long exserted, fused in 2 pairs or rarely all 4 attached; filaments mostly darker in the upper half and ranging from reddish to purplish and then to white at base, sparsely glandular pubescent to glabrous or with few sessile glands in lower part, anthers dark maroon, purple, yellow, white and purple, or greyish purple; anterior filaments inserted at $26-48 \mathrm{~mm}$ from corolla base which is $50-72 \%$ of corolla length, 
filaments $25-50 \mathrm{~mm}$ long, anthers 3-5.6 $\times 0.9-2.3 \mathrm{~mm}$; posterior filaments inserted at $32-50 \mathrm{~mm}$ from corolla base which is $58-82 \%$ of corolla length, filaments $25-42$ mm long, anthers 2.5-5 × 1-2.1 mm; staminode 0.7-11 mm long. Pollen ochre or yellow. Disk 0.5-2.5 mm high, a simple annular ring, 5-dentate, 5-crenate or strongly 5-lobed. Pistil 15.5-80 mm long; stipe 3-26 mm long, glabrous; ovary green or cream, 8-38 mm long, glabrous or with sessile glands; style cream or white, 4.5-41 mm long, glandular pubescent to glabrous. Capsule 15.7-42 cm long, 2-5 mm wide. Seed grain $0.8-2 \times 0.2-0.5 \mathrm{~mm}$, warty, bubble cells absent; apical appendage a filiform hair, 16-34 mm long; hilar appendage a single filiform hair, 13.5-35 mm long; appendages papillose.

Distribution. Myanmar, Thailand, Cambodia, Laos, Vietnam, Peninsular Malaysia (Kedah). In Peninsular Malaysia only recorded from Gunung Jerai in Kedah.

Habitat and ecology. Across its distribution it has been recorded in a wide variety of habitats including primary or disturbed evergreen, mixed deciduous or mossy forest at 10-1500 m altitude. The ecology and altitude for this species on Gunung Jerai was not recorded on the specimens.

Provisional IUCN conservation assessment. Least Concern (LC). This species has a widespread distribution and is common in parts of its range, particularly in Thailand. In Peninsular Malaysia it has only been collected a few times and at only one locality, Gunung Jerai. Its current status there needs to be clarified.

Peninsular Malaysian specimens examined. PENINSULAR MALAYSIA: Kedah: Gunung Jerai, 16 Oct 1976, Stone, B.C.M. 12758 (KLU); ibidem, 18 Nov 1977, Lo, Y.N. \& Mahmud 183 (KLU); ibidem, 23 Nov 1999, Kiew, R. RK4862 (KEP, SING).

Notes. This is a widespread and variable species but in Peninsular Malaysia has only been collected on Gunung Jerai. The description above includes the species from throughout its range as the very few Malaysian specimens are inadequate to write a full description. The material seen from Malaysia has a long and narrow calyx tube with small lobes but elsewhere in the distribution of Aeschynanthus fulgens this species is known to be very variable, especially in this character.

6. Aeschynanthus Iongicaulis Wall. ex R.Br., Cyrtandreae 116 (1839); Steudel, Nomencl. Bot. ed. 2, 1: 32 (1840); Brown in Bennett, Pl. Jav. Rar. 116 (1840); A.DC., Prod. 9: 262 (1845); Miquel, Fl. Ned. Ind. 2: 719 (1858); C.B.Clarke in A.DC. \& C.DC., Monogr. Phan. 5(1): 19 (1883); C.B.Clarke in A.DC. \& C.DC., Monogr. Phan. 5(1): 19 (1883); Barnett, Fl. Siam. 3 (3): 201 (1962); Burtt \& Woods, Notes Roy. Bot. Gard. Edinburgh 33: 481 (1975), pro parte; Chin, Gard. Bull. Singapore 32: 147 (1979); Li, Acta Bot. Yunnan. 5 (1): 36 (1983); Wang, Fl. Reipubl. Popularis Sin. 69: 526 (1990); Turner, Gard. Bull. Singapore 47: 243 (1997 ['1995’]); Wang, Pan, Li, 
Weitzman \& Skog, Fl. China 18: 385 (1998); Burtt, Thai Forest Bull., Bot. 29: 83 (2001), pro parte; Smitinand, Thai Pl. Names ed. 2, 14 (2001); Kress et al., Checkl. Myanmar 261 (2003). - Trichosporum longicaule (Wall. ex R.Br.) Kuntze, Revis. Gen. Pl. 478 (1891). - TYPE: Burma, Chappedong, Wallich, N. 888 (lectotype K [K000096741], designated by Middleton (2007); isolectotypes BM [BM000883859], G-DC, K-W). (Fig. 8, 9)

Aeschynanthus griffithii R.Br., Cyrtandreae 115 (1839); Brown in Bennett, Pl. Jav. Rar. 115 (1840); A.DC., Prod. 9: 261 (1845); Miquel, Fl. Ned. Ind. 2: 719 (1858); C.B.Clarke in A.DC. \& C.DC., Monogr. Phan. 5(1): 24 (1883); Clarke in Hooker, Fl. Brit. Ind. 4: 339 (1884); Kress et al., Checkl. Myanmar 261 (2003). - Trichosporum griffithii (R.Br.) Kuntze, Revis. Gen. Pl. 477 (1891). - TYPE: Burma, Tenasserim Division, Tavoy, Griffith, W. s.n. (holotype BM [BM000883870]).

Aeschynanthus zebrinus Van Houtte, Hort. Vanhoutt. 1(2): 42 (1846). - TYPE: Not located.

Aeschynanthus marmoratus T.Moore, Paxton's Fl. Gard. 3: 56 (1852); C.B.Clarke in A.DC. \& C.DC., Monogr. Phan. 5(1): 38 (1883); Ridley, J. Linn. Soc. Bot. 32: 500 (1896); Ridley, J. Straits Branch Roy. Asiat. Soc. 44: 13 (1905); Ridley, J. Asiat. Soc. Bengal 74 (2): 732 (1909); Ridley, Fl. Mal. Pen. 2: 498 (1923); Barnett, Fl. Siam. 3 (3): 202 (1962). - Trichosporum marmoratum (T.Moore) T.Moore ex Kuntze, Revis. Gen. Pl. 478 (1891). - TYPE: Cultivated specimen, October 1851, Moore, T. s.n. (holotype K [K000831883]).

Epiphyte with arching and pendulous branches; stems grey-brown or grey, glabrous. Leaves opposite; petiole 2-14 mm long, glabrous; blade fleshy, background mid to dark green above but variegated with much paler venation, same but paler beneath, elliptic, 1.9-12.2 × 0.3-4 cm, 2.2-9 times as long as wide, apex caudate to acuminate, base cuneate to acute, margin with few minute teeth but appearing more or less entire, glabrous above and beneath, 5-6 pairs of secondary veins, weakly visible, tertiary venation obscure. Inflorescences terminal, peduncle absent; pedicels 9.5-11 mm long, green suffused purple, glabrous. Calyx of separate lobes free to base, orange, purple or green flushed maroon-purple, glabrous or with sparse glandular hairs; lobes narrowly elliptic to linear, erect, $8-18 \times 0.8-2.4 \mathrm{~mm}$, apex acute to acuminate. Corolla $20.5-31 \mathrm{~mm}$ long, externally tube green, yellowish green or green with flushes of orange, purple or brown, lobes purple or maroon with an orange or yellow margin or all orange, internally tube dark red, maroon or green, lobes maroon, purple or red with a yellow or orange margin; upper lobes ovate or orbicular, not spreading or reflexed, 2.5-3.8 $\times 2.4-4.5 \mathrm{~mm}$, sinus 2.2-4.5 mm deep, apex rounded; lateral lobes orbicular or oblique ovate, not spreading or reflexed, 2-3.9 $\times 3.3-4.8 \mathrm{~mm}$, rounded; lower lobe ovate or squarish, not spreading or reflexed, $2.5-5.8 \times 3.6-4.2 \mathrm{~mm}$, apex rounded, outside glabrous except for ciliate lobes, to densely glandular puberulent, inside with five dense tufts of multicellular hairs near base. Stamens long exserted; filaments 

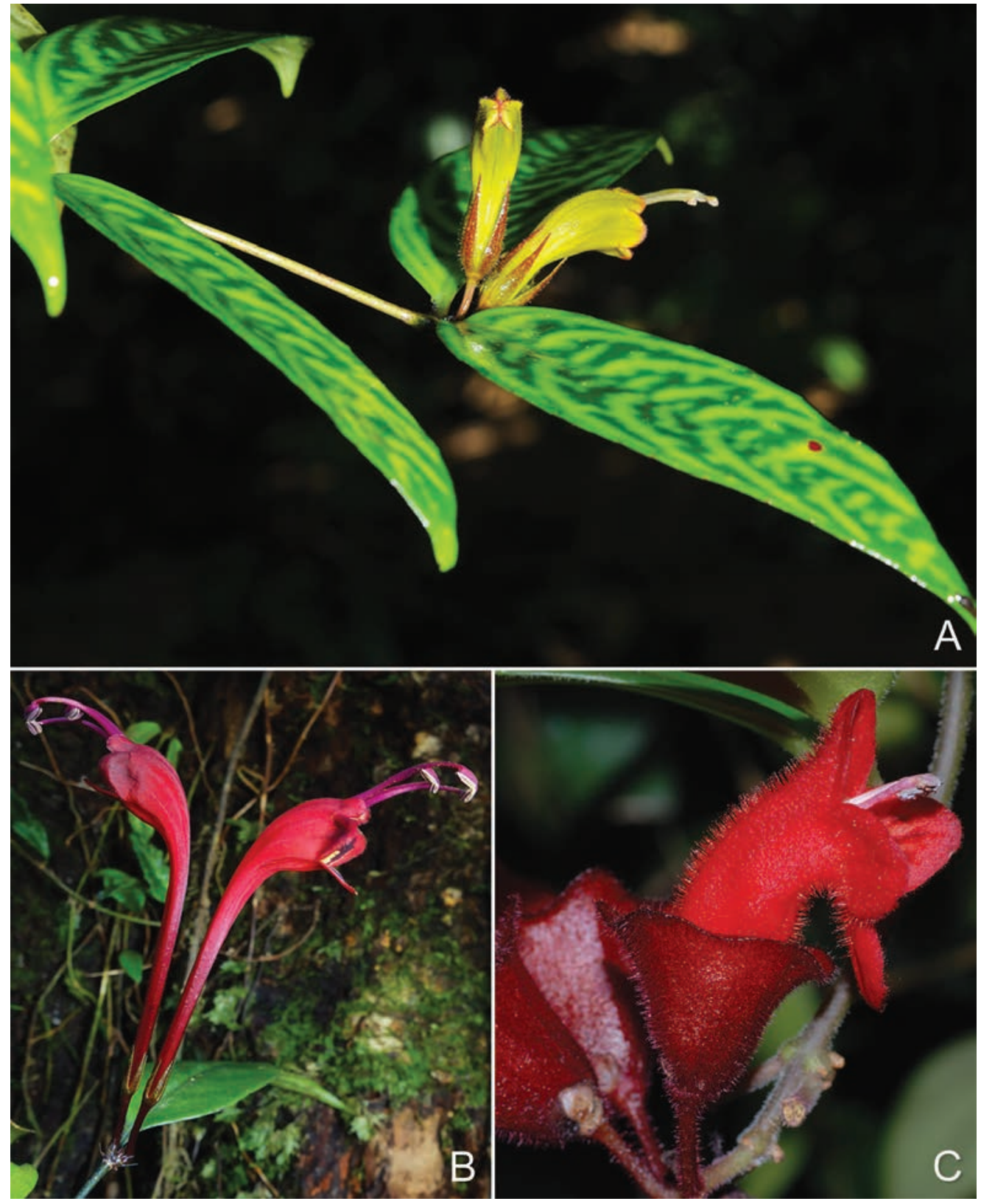

Fig. 8. A. Aeschynanthus longicaulis Wall. ex R.Br. B. Aeschynanthus longiflorus (Blume) A.DC. C. Aeschynanthus obconicus C.B.Clarke. (Photos: A, Preecha Karaket; B, Jana LeongŠkorničková; C, David Middleton)

green or yellow, with glandular hairs; anthers pale brown or grey; anterior filaments inserted at $12-19.5 \mathrm{~mm}$ from corolla base which is $48-52 \%$ of corolla length, anthers 2.6-3.2 × 1-1.2 mm; posterior filaments inserted at $12.5-21 \mathrm{~mm}$ from corolla base 


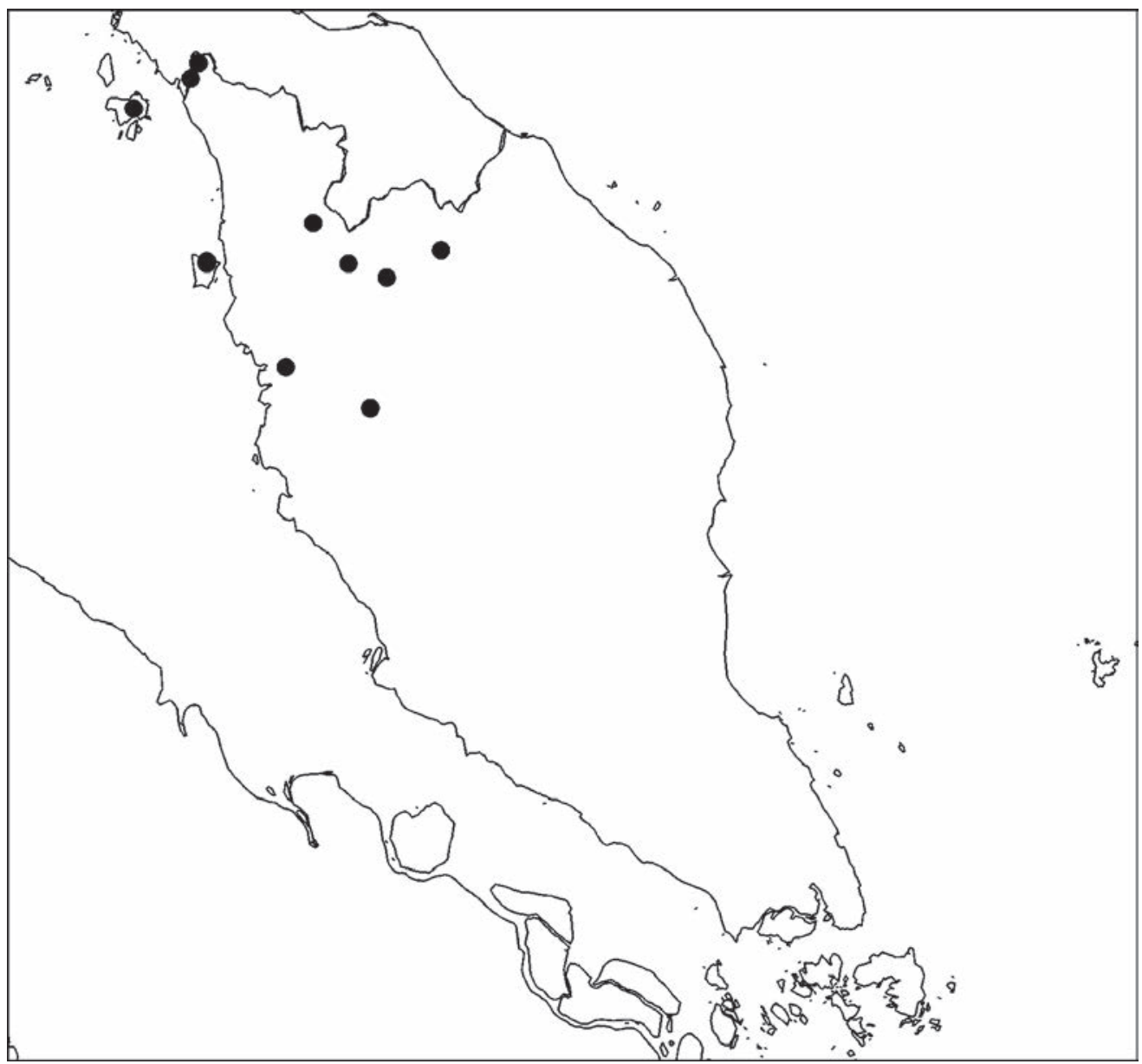

Fig. 9. Distribution of Aeschynanthus longicaulis Wall. ex R.Br. in Peninsular Malaysia (•).

which is $50-55 \%$ of corolla length, filaments $23-27.5 \mathrm{~mm}$ long, anthers $1.8-2.8 \times$ 0.7-1.3 mm; staminode 0.9 mm long; pollen greenish grey. Disk 0.9-1.3 mm high, a simple annular ring. Pistil 14-45 mm long; stipe 1.5-4 mm long, glabrous; ovary 5.5-12 mm long, glabrous or with sessile glands; style yellow or green flushed purple towards apex, 7-31 mm long, glandular pubescent; stigma purple or yellow. Capsule 5.4-37 cm long, 1.8-3.2 mm wide. Seed grain 1.6-2.2 × 0.1-0.6 mm, warty, bubble cells absent; apical appendage a filiform hair, c. $22.5 \mathrm{~mm}$ long; hilar appendages of many (13-25) filiform hairs, 10-25 mm long; appendages papillose.

Distribution. Eastern Myanmar, western and southern Thailand, Peninsular Malaysia (Kedah, Kelantan, Penang, Perak, Perlis).

Habitat and ecology. In primary and secondary lowland forest, reaching to upper dipterocarp forest, sometimes on limestone, at 360-1150 m in Peninsular Malaysia. 
Most Malaysian material does not have good habitat and altitude notes but in Thailand it has also been collected in rubber plantations.

Provisional IUCN conservation assessment. Least Concern (LC). This species has a wide distribution and has been recorded from a variety of habitats. However, it should be noted that it has been collected rather infrequently in Peninsular Malaysia in recent years and only once since 2000 so its status should be monitored.

Peninsular Malaysian specimens examined. PENINSULAR MALAYSIA: Kedah: Langkawi, Gunung Raya, 880 m, 13 Sep 1988, Tay, E.P. 125 (SING); Weng Road near Baling, 2 Nov 1929, Best, G.A. 21255 (SING); Kelantan: Jeli, Upper Sungai Pergau, 25 Sep 1986, Latiff, A. et al. ALM1813 (PSU, UKMB); Penang: Government Hill, 760 m, Sep 1889, Curtis, C. 2142 (SING); ibidem, Mar 1889, Ridley, H.N. 1700 (BM, SING); Penang Hill, 760 m, 8 Sep 1982, Kochummen, K.M. FRI29332 (K, KEP, L); Perak: Gunong [Gunung] Chabang, Scortechini, B. 35 (SING); Ulu Temengor, Jul 1909, Ridley, H.N. 14280 (BM, SING); Grik, 19 Jun 1924, Burkill, H.M. \& Md Haniff 12542 (SING); Taiping, Bukit Larut, 1150 m, 12 May 2009, Yao, T.L. FRI57961 (KEP); ibidem, Heng, H.P. et al. HP10 (SINU); Perlis: Kaki Bukit, 1962, Cultivated C4877 (E); Bukit Bintang Forest Reserve, 360 m, 25 Apr 1962, Burtt, B.L. \& Woods, P.J.B. B1739 (E).

Notes. This species is difficult to distinguish from Aeschynanthus fecundus when not in flower. The name has also been applied widely to species from other parts of Asia that are now recognised as distinct taxa such as Aeschynanthus membranifolius and A. sinolongicalyx W.T.Wang. These species all share characteristic variegated leaves. Flowering material is needed to assess whether Aeschynanthus longicaulis occurs further north into northern Thailand than previously suspected or whether other related species rather have the wider distribution.

The name Aeschynanthus marmoratus, originally described from cultivated material, was long applied to the material from Peninsular Malaysia but the type material is indistinguishable from that of Aeschynanthus longicaulis.

7. Aeschynanthus longiflorus (Blume) A.DC., Prod. 9: 262 (1845); Hooker, Bot. Mag. 73: t.4328 (1847); Zollinger, Syst. Verz. 3: 57 (1855); Miquel, Fl. Ned. Ind. 2: 717 (1858); C.B.Clarke in A.DC. \& C.DC., Monogr. Phan. 5(1): 32 (1883); Ridley, J. Linn. Soc. Bot. 32: 499 (1896); Ridley, J. Straits Branch Roy. Asiat. Soc. 44: 14 (1905); Ridley, J. Asiat. Soc. Bengal 74 (2): 734 (1909); Ridley, Fl. Mal. Pen. 2: 498 (1923); Backer \& Bakhuizen van den Brink, Fl. Java 2: 524 (1965); Turner, Gard. Bull. Singapore 47: 243 (1997 ['1995’]); Burtt, Thai Forest Bull., Bot. 29: 83 (2001); Smitinand, Thai Pl. Names ed. 2, 15 (2001); Middleton, Edinburgh J. Bot. 64: 400 (2007). - Lysionotus longiflorus Blume, Bijdr. Fl. Ned. Ind. 766 (1826). - Trichosporum longiflorum (Blume) Kuntze, Revis. Gen. Pl. 478 (1891); Merrill, Contr. Arnold Arbor. 8: 151 (1934). - TYPE: Indonesia, Java, Blume, C.L. 247 (lectotype L [Leiden number 903,307-102], designated by Middleton (2007); isolectotypes L [2 sheets]). (Fig 8, 10) 
Aeschynanthus perakensis Ridl., J. Linn. Soc. 32: 499 (1896); Ridley, J. Straits Branch Roy. Asiat. Soc. 44: 15 (1905); Ridley, J. Asiat. Soc. Bengal 74 (2): 734 (1909); Ridley, Fl. Mal. Pen. 2: 498 (1923); Henderson, Malay. Wild. Fl. Dicot. 340 (1959); Turner, Gard. Bull. Singapore 47: 243 (1997 ['1995’]). - TYPE: Peninsular Malaysia, Perak, Taiping, Bukit Larut, 1891, Ridley, H.N. s.n. (lectotype SING [SING0035528], designated by Middleton (2007)).

Epiphyte with arching stems; stems glabrous. Leaves opposite; petiole 3-16 mm long, glabrous; blade slightly fleshy or coriaceous, dark to mid green above, pale green beneath, ovate or elliptic, not variegated, 3.7-17.5 × 1.6-6.7 cm, 1.5-6.1 times as long as wide, apex acuminate to caudate, base rounded to cuneate, glabrous above and beneath, margin entire, secondary veins obscure to weakly visible, 3-8 pairs, tertiary venation obscure. Inflorescences terminal, 2-5-flowered, peduncle absent, bracts linear, 3-6 mm long; pedicels 11-16 mm long, brownish green or purplish, glabrous. Calyx of separate lobes free to base, often differing quite substantially in length even within a single flower, green to dark red or purplish, with sparse glandular hairs or glabrous, sometimes with ciliate lobes or with just a few hairs only on very tips, 4.8$23 \mathrm{~mm}$ long; lobes linear, narrowly triangular, narrowly ovate or oblong, erect, 4.8-23 $\times 1-2.2 \mathrm{~mm}$, apex obtuse to acuminate. Corolla 43-90 mm long, tube narrow at base; externally tube bright red or dark red, lobes bright red or dark red, internally tube cream-coloured, lobes bright red on upper 2 lobes and bright red at margin with a darker W-shaped line on lower 3 lobes; upper lobes orbicular, oblong or ovate, not spreading or reflexed, 3.2-8.1 $\times 3.2-5 \mathrm{~mm}$, sinus 3-5.8 $\mathrm{mm}$ deep, apex rounded; lateral lobes oblique ovate or deltoid, not spreading or reflexed, 3.2-7.5 $\times 5.5-9 \mathrm{~mm}$, apex rounded; lower lobe ovate, not spreading or reflexed, 6-12 $\times 4-6.2 \mathrm{~mm}$, apex rounded; outside glabrous to sparsely glandular puberulent, usually with ciliate lobes, inside with sparse glandular hairs throughout, sometimes very sparsely so, and sessile glands below lobes. Stamens long exserted, fused in 2 pairs; filaments reddish, purple or pink, with small glandular hairs, anthers pale pink; anterior filaments inserted at $49-60 \mathrm{~mm}$ from corolla base which is $72-77 \%$ of corolla length, filaments $29-50 \mathrm{~mm}$ long, anthers 3.3-5 × 1-2 mm; posterior filaments inserted at 50-64 mm from corolla base which is $77-81 \%$ of corolla length, filaments $23-37 \mathrm{~mm}$ long, anthers $2.4-3.8 \times$ 0.9-2 mm; staminode 0.7-5 mm long. Disk 1-1.5 mm high, 5-crenate. Pistil 45-83 mm long; stipe 13-20 mm long, glabrous to sparsely minutely papillose; ovary 19-29 mm long, minutely papillose puberulent or with few sessile glands; style purple or purplish pink, 13-34 mm long, glandular pubescent to papillose, sometimes sparsely so. Capsule 21-57 cm long, 3.5-4 mm wide. Seed grain 1-1.8 × 0.3-0.4 mm, warty, bubble cells absent; apical appendage a filiform hair, 15-24 mm long; hilar appendage a single filiform hair, 15-24 mm long; appendages papillose.

Distribution. Peninsular Thailand, Peninsular Malaysia (Kelantan, Negeri Sembilan, Pahang, Penang, Perak, Selangor, Terengganu), Sumatra, Java, Borneo. 


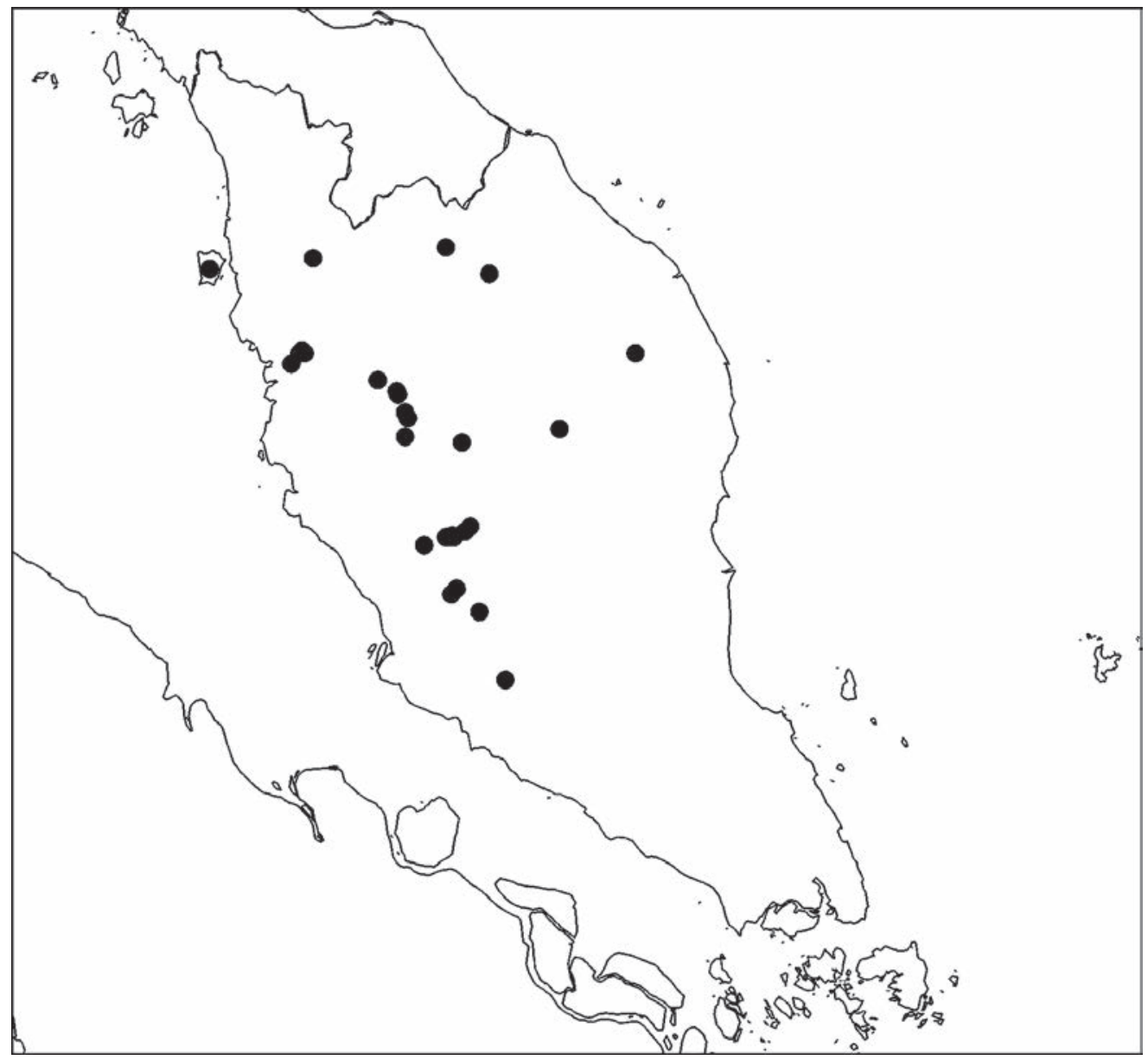

Fig. 10. Distribution of Aeschynanthus longiflorus (Blume) A.DC. in Peninsular Malaysia (•).

Habitat and ecology. This species is recorded most commonly in lower montane forest up to $1535 \mathrm{~m}$ altitude and occasionally from lowland or hill dipterocarp forest. However, in Peninsular Malaysia it is also recorded from as low as 90 m altitude.

Provisional IUCN conservation assessment. Least Concern (LC). This species is widely distributed in the region.

Additional Peninsular Malaysian specimens examined. PENINSULAR MALAYSIA: Kelantan: Jeli, Upper Sungai Pergau, 25 Sep 1986, Latiff, A. et al. ALM1789 (PSU, UKMB); Kuala Krai, Stong Tengah FR, Sungai Kenerong, 564 m, 4 Apr 2009, Rosdi, M., Phoon, S.N., Ong, P.T. \& Imin, K. FRI66272 (KEP); Negeri Sembilan: Jelebu, Bkt Tangga, G. Telapak Burok, 9 Apr 2008, Phoon, S.N. \& Imin, K. FRI60648 (KEP); Pahang: Tahan River, 1520 m, 1891, Ridley, H.N. 2167 (K, SING); Fraser's Hill, Nov 1976, Keng, H. et al. 4755 (SINU); ibidem, 1210 m, 18 Apr 1955, Purseglove, J.W. P4164 (E, K, L, SING); ibidem, 1210-1320 m, 16-30 Sep 1922, Burkill, H.M. \& Holttum, R.E. 7888 (K, SING); ibidem, 1500 m, 16 Apr 
1962, Burtt, B.L. \& Woods, P.J.B. B1644 (E, SING); ibidem, 13 Mar 1986, Anthony, S. SA481 (KEP); ibidem, 1270 m, 4 Oct 1961, Burkill, H.M. HMB2812 (SING); ibidem, 1210 m, 20 Mar 1929, Holttum, R.E. 21528 (SING); ibidem, 25 Oct 1959, Mitchell s.n. (SING); ibidem, 27 Aug 1991, Kiew, R. RK3293 (SING); Fraser’s Hill, Rompin Trail, 1218 m, 26 Oct 2010, Julius, A. FRI73605 (K, KEP, SING); Fraser's Hill, Bishop’s Trail, 1275 m, 31 Mar 2007, Kiew, R., Lindsay, S. \& Middleton, D.J. FRI57497 (KEP); Fraser's Hill, Raub, 1260 m, 21 Mar 1994, Perumal, B., Gan, C.L., Shahril, K.Z., Angan, A. \& Bedul FRI41512 (KEP); Fraser's Hill, Tras Valley, 1090 m, 27 Sep 1922, Burkill, H.M. 8859 (SING); ibidem, 1150 m, 25 Mar 1929, Holttum, R.E. s.n. (SING); 3rd Mile Genting Highlands Road, 910 m, 2 Oct 1970, Kochummen, K.M. FRI16215 (KEP); Cameron Highlands, 48th Mile Blue Valley, 23 Mar 1963, Mohd. Kassim bin Rajab 488 (KLU); Cameron Highlands, Gunung Berembun, Nov 1908, Ridley, H.N. 13603 (SING); Cameron Highlands, Nov 1940, Quaife s.n. (SING); Telom, Nov 1908, Ridley, H.N. s.n. (SING); Cameron Highlands, G. Siku FR, 1430 m, 21 Jan 2010, Mohd Hairul, M.A., Ong, P.T., Siti Munirah, M.Y. \& Kueh, H.L. FRI69946 (KEP); Cameron Highlands, G. Siku FR, Beside the road to G. Siku FR, 1535 m, 25 Oct 2007, Imin, K. \& Kueh, H.L. et al. FRI58561 (KEP); Cameron Highlands, Robinson's Falls, 24 Mar 2007, Julius, A., Middleton, D.J. \& Lindsay, S. et al. FRI57479 (KEP); Penang: unknown s.n. (E); Perak: Gunung Korbu, 1210-1300 m, 11 Mar 1913, Robinson, H.C. s.n. (K); Taiping, 910-1210 m, Nov 1885, King's Collector 8314 (K); Taiping, Bukit Larut, 790 m, 18 Sep 1949, Sinclair, J. \& Kiah SFN38800 (E, K, SING); ibidem, 1270 m, 25 Nov 1980, Keng, H. et al. 85 (SINU); ibidem, Dec 1902, Ridley, H.N. 11447 (K, SING); ibidem, 1060-1210 m, Dec 1884, King’s Collector 7022 (K, SING); ibidem, 18 Sep 1949, Sinclair, J. 6198 (E); ibidem, Anderson, J.W. 95 (SING); ibidem, 910 m, 29 Oct 1968, Smith, G. 443 (KLU); ibidem, 1290-1330 m, 8 Mar 1939, Spare, G.H. 2114 (SING); Caulfield's Hill, Wray, L. 625 (K, SING); Gunung Bintang, Bukit Kuala Bintang, 18 Apr 1928, Md Haniff 21088 (SING); Taiping, Gunung Hijau, Sep 1889, Curtis, C. s.n. (SING); ibidem, 1300 m, 20 Oct 1992, Saw, L.G. FRI37667 (KEP); Selangor: Genting Highlands, Gunung Bunga Buah, 1090 m, 5 May 1999, Chua, L.S.L. FRI 40785 (KEP); Semangkok Pass, 1909, Ridley, H.N. s.n. (SING); Gombak, Gunung Bungah Buah, Trail to Gunung Bunga Buah, 1250 m, 31 Oct 2010, Yao, T.L., Kiew, R. \& Wen, J. FRI65495 (E, KEP); Hulu Langat, Gn. Nuang, Above Pacat Camp, 1418 m, 12 Dec 2013, Yao, T.L., Imin, K., Mohd Nazri, A. \& Kueh, H.L. FRI77335 (KEP); Terengganu: Ulu Brang, Gunong [Gunung] Padang, Near Kampong Lallang, 90 m, 16 Sep 1969, Whitmore, T.C. FRI12601 (K, KEP, L, SING).

Notes. This species is most similar to Aeschynanthus speciosus, particularly those forms with redder flowers, but is easily distinguished by the opposite leaves of $A$. longiflorus and the whorled leaves of A. speciosus.

The calyx pubescence character used by Ridley (1923) to distinguish Aeschynanthus perakensis from A. longiflorus does not hold up to scrutiny and this character is very variable in the species. As noted by Middleton (2007) the type of Aeschynanthus perakensis is cited by Ridley as 'Perak, on Gunong Hijan; Thaiping Hills, at an altitude of 5,000 feet'. No Ridley material has been found which has this locality information on the label and only one specimen has been found which could possibly be original material. This specimen is labelled 'Maxwell's Hill', now known as Bukit Larut of which Gunung Hijau (the modern name for Gunong Hijan) is a part. This specimen was lectotypified by Middleton (2007). A second Ridley specimen from the area cannot be counted as original material as Ridley explicitly says he has seen no fruiting material and the specimen has fruits. 
8. Aeschynanthus obconicus C.B.Clarke in A.DC. \& C.DC., Monogr. Phan. 5(1): 50 (1883); Clarke in Hooker, Fl. Brit. Ind. 4: 343 (1884); Ridley, J. Linn. Soc. Bot. 32: 501 (1896); Ridley, J. Asiat. Soc. Bengal 74 (2): 737 (1909); Ridley, Fl. Mal. Pen. 2: 500 (1923); Turner, Gard. Bull. Singapore 47: 243 (1997 ['1995’]). Trichosporum obconicum (C.B.Clarke) Kuntze, Revis. Gen. Pl. 478 (1891). - TYPE: Peninsular Malaysia, Selangor, Klang, 26 December 1878, Beccari, O. 36 (holotype FI [FI013085]). (Fig. 8, 11)

Epiphytic, stems mostly hanging, green, mostly glabrous, occasionally sparsely puberulent only around nodes. Leaves opposite; petiole 3-10 mm long, glabrous or rarely sparsely puberulent; blade coriaceous, ovate or elliptic, 2.5-10.7 × 0.9-6 cm, 1.5-3.1 times as long as wide, apex acuminate or acute, base cuneate to rounded, mid green above, paler beneath, not variegated, glabrous above and beneath, occasionally with few marginal hairs at margin base, margin entire, sometimes undulate, 4-7 pairs of secondary veins, weakly visible or obscure, tertiary venation obscure. Inflorescences axillary or subterminal, 1-6-flowered; peduncles $4 \mathrm{~mm}$ long; bracts elliptic, 1.3-4 mm long; pedicels 6-12 mm long, dark red, sparsely to densely eglandular puberulent. Calyx with a tube and short and broad lobes or barely any lobes at all, the whole broadly cup-shaped, dark red or dark red at base and becoming bright red higher, sometimes purplish brown, outside densely to sparsely pubescent with red hairs, these varying degrees of eglandular and glandular, very rarely glabrous, inside sparsely pubescent to glabrous, total length 8-19 mm long; tube 7-15 mm long which is 74$94 \%$ of total length, 15-27 mm wide at top of tube; lobes semicircular or a weak curve of the upper rim, erect, spreading or recurved, 0.7-6.5 mm long, 9-16 mm wide, apices rounded. Corolla 23-35 mm long, tube broad at base, externally bright to dark red, internally cream, lobes externally bright red with cream at base of lower 3 lobes, internally red with cream and dark red markings on lower 3 lobes; upper lobes ovate, oblong or slightly falcate, spreading, 4.2-10 × 1.7-6 mm, sinus between lobes 3-5 mm deep, apices rounded; lateral lobes ovate to oblong, spreading to reflexed, 4.2-12.5 $\times$ 4.2-9.8 mm, apices rounded; lower lobe ovate or elliptic, spreading, 5-11.4 × 5.6-6.7 $\mathrm{mm}$, apex rounded; outside densely pubescent or rarely glabrous except for ciliate lobes, inside with scattered short glandular hairs with robust base, sessile glands at lobe sinuses. Stamens not or only slightly exerted, fused in 2 pairs, filaments cream to bright red, papillose, anthers cream or yellow; anterior filaments inserted at 9-15 $\mathrm{mm}$ from corolla base which is $32-58 \%$ of corolla length, filaments $15-18.5 \mathrm{~mm}$ long, anthers $2.4-2.7 \times 1.2-1.5 \mathrm{~mm}$; posterior filaments inserted at $12-17 \mathrm{~mm}$ from corolla base which is $43-63 \%$ of corolla length, filaments $12.8-16 \mathrm{~mm}$ long, anthers $1.6-2.5$ $\times$ 0.9-1.6 mm; staminode c. $1 \mathrm{~mm}$ long. Disk $0.5-1 \mathrm{~mm}$ high, a simple annular ring. Pistil 18.5-36.5 mm long; stipe 6-13 mm long, with few sessile glands or glabrous; ovary cream to pale green, 9.5-26 mm long, with sessile glands; style 2-7.5 mm long, glandular pubescent, sometimes these sparse. Capsules $18-41 \mathrm{~cm}$ long, $2.8 \mathrm{~mm}$ wide. Seed grain $0.8 \mathrm{~mm} \times 0.3 \mathrm{~mm}$, papillose, bubble cells present at base of hilar appendage; apical appendage a filiform hair, $12.5 \mathrm{~mm}$ long; hilar appendage a single filiform hair, $10 \mathrm{~mm}$ long; appendages not papillose. 


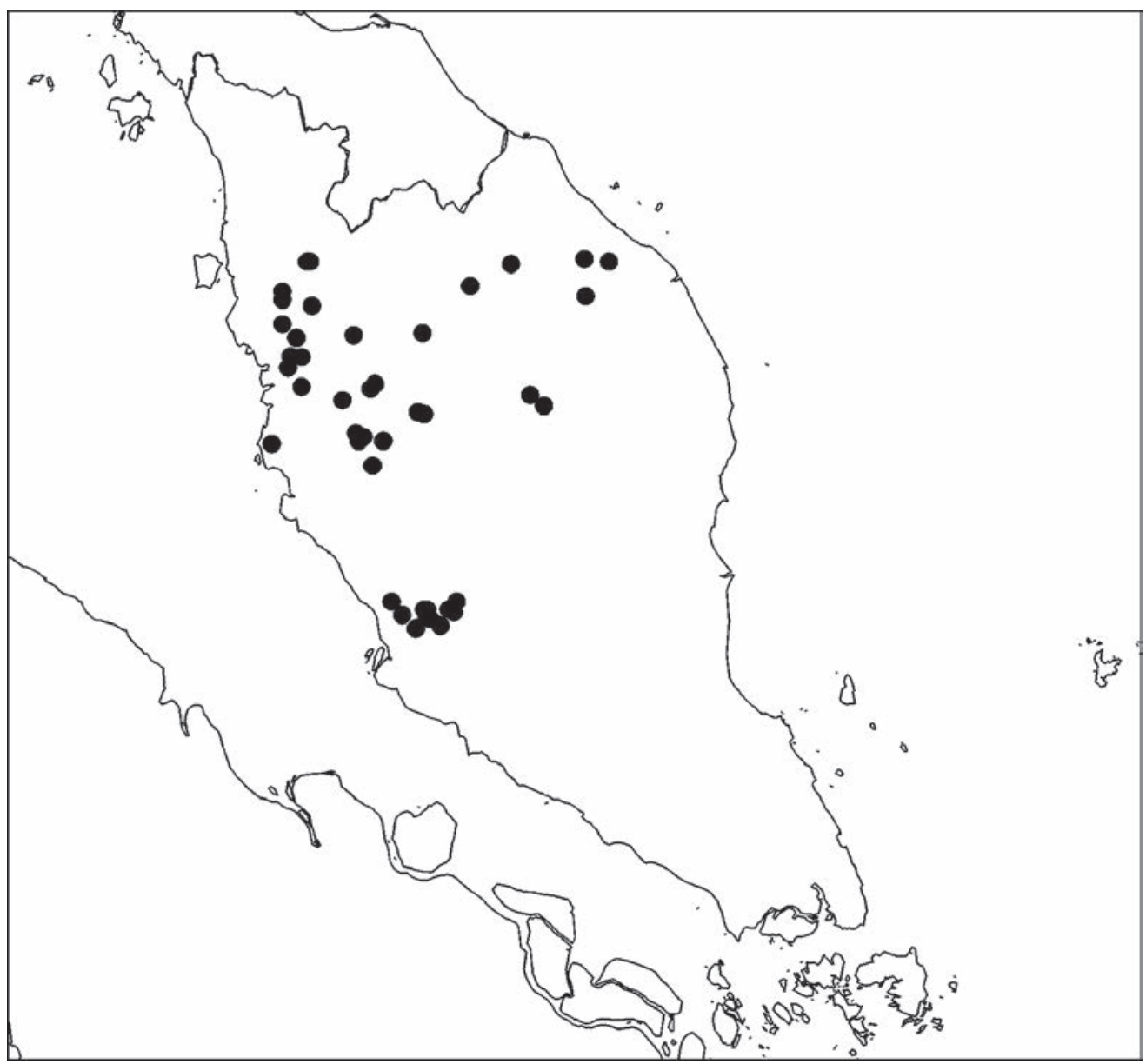

Fig. 11. Distribution of Aeschynanthus obconicus C.B.Clarke in Peninsular Malaysia (•).

Distribution. Peninsular Malaysia (Kedah, Kelantan, Kuala Lumpur, Pahang, Perak, Selangor, Terengganu), Sumatra, Borneo.

Habitat and ecology. Epiphytic in lowland dipterocarp forest, often on trees by rivers, occasionally in lower montane forest, at 0-1300 m altitude.

Provisional IUCN conservation assessment. Least Concern (LC). This species is widespread and occurs in a variety of forest types.

Additional Peninsular Malaysian specimens examined. PENINSULAR MALAYSIA: unknown loc., 21 Nov 1932, unknown collector FMS17068 (K, KEP); unknown state, Belau Tajor, Wray, L. 1772 (SING); Kedah: Gunong [Gunung] Bintang, 8 Apr 1968, Sidek bin Kiah S.277 (L, SING); Kelantan: Kpg. Bihai, Sg. Kerchit, 13 Feb 2003, Kiew, R. RK5239 (SING); Kuala Krai, Stong Tengah FR, Sg. Kenerong, 4 Apr 2009, Imin, K., Phoon, S.N., Ong, P.T. \& Rosdi, M. et al. FRI68188 (KEP); Kuala Lumpur: Feb 1890, Curtis, C. s.n. (SING); Pahang: 
Sungai Telom, 450 m, 24 Aug 1930, Kiah 23943 (NY, SING); Puku, Kuala Teku, 150 m, 21 Feb 1921, Seimund, E. 459 (SING); Kuala Terla Forest Reserve, Trail from Kg. Sg. Jarik, 540 m, 23 Mar 2007, Julius, A. FRI57451 (KEP, SING); Gunung Tahan, Teku, 21 Jun 1922, Md Haniff \& Md Nur 8046 (SING); Taman Negara, Sg. Tahan, Kem Teku, 283 m, 12 May 2008, Lim, C.L. \& Kueh, H.L. FRI64886 (KEP); Perak: unknown loc., Jan 1886, King's Collector 10179 (SING); Gunong [Gunung] Inas, Sira Rimau, 27 Dec 1899, Yapp, R.H. 547 (CGE); Larut Matang, Bkt. Larut, Sungai Bt. Tugoh, 72 m, 28 Jul 2011, Imin, K. \& Asmarayani, H.S. FRI76004 (KEP); Taiping, Bukit Larut, 610 m, 1901, Curtis, C. s.n. (SING); Taiping, Dec 1902, Ridley, H.N. s.n. (SING); ibidem, 25 Aug 1971, Kochummen, K.M. FRI16427 (KEP); ibidem, 450 m, Sep 1889, Curtis, C. s.n. (SING); ibidem, Jul 1881, King's Collector 2012 (SING); ibidem, 1000-1300 m, Dec 1882, King's Collector 3641 (SING); ibidem, Jul 1881, King's Collector 2049 (SING); ibidem, 9 Mar 1924, Burkill, H.M. \& Md Haniff 12798 (SING); ibidem, 4 Mar 1965, Hardial, S. \& Samsuri Ahmad 290 (SING); ibidem, Mar 1882, King's Collector 2849 (SING); ibidem, Mar 1892, Ridley, H.N. 2905 (BM, SING); ibidem, 760-790 m, 16 Sep 1949, Sinclair, J. \& Kiah SFN38805 (E, SING); ibidem, 610 m, 25 Aug 1971, Whitmore, T.C. FRI20392 (K, KEP, L, SING); Gunong [Gunung] Bubu, 600 m, 18 Aug 1966, Ding Hou 657 (K, KEP, L, SING); ibidem, 610 m, 16 Aug 1966, Chew, W.-L. CWL1213 (K, KEP, SING); Kampong Sekam, 510 m, 19 Oct 1982, Avé, W. 165 (K, L); Dinding, 1888, Curtis, C. 1388 (K, SING); Kinta, Kinta Dam, Trail to G. Korbu, 450 m, 10 Jun 2010, Imin, K. et al. FRI71615 (K, KEP); Gunung Korbu, 610 m, 20 Mar 1913, Robinson, H.C. s.n. (K); Kuala Dipang Forest Reserve, 30-210 m, 19 Feb 1976, Sidek bin Kiah SK515 (C, KEP, SING); Sg. Siput, Chior Forest Reserve, Sungai Legap, 180 m, 9 Oct 1967, Ng, F.S.P. FRI5813 (KEP); Chenderiang, Gunong [Gunung] Bujang Melaka, Sungai Rias, 610 m, 12 Feb 1975, Shah, M., Ahmad \& Mahmud MS3407 (C, KEP, SING); Bujang Melaka, Aug 1898, Curtis, C. 3335 (SING); Batu Kurau, Curtis, C. 2990 (SING); Pondok Tanjong Forest Reserve, 14 Feb 1939, Spare, G.H. SFN36226 (SING); Sungei Merbau, 10 Dec 1929, Symington, C.F. 21390 (SING); Bleinja [?Belanjar], Wray, L. 149 (SING); Scortechini, B. 46a (SING); Ijok Forest Reserve, 11 Feb 1939, Aziz Budin 48644 (KEP [2 sheets]); Dinding, Curtis, C. s.n. (SING); Tapah, Nov 1908, Ridley, H.N. 14063 (SING); Bujang Melaka, Sep 1898, Ridley, H.N. s.n. (SING); Dinding, 1892, Curtis, C. s.n. (SING); Gunung Bintang, Bukit Kuala Bintang, 17 Apr 1928, Md Haniff 21074 (SING); Bintang Hijau FR, Sg. Tebing Tinggi, 317 m, 26 Aug 2009, Chan, Y.M., Norzamli, A. \& Norazmi, A. FRI70603 (KEP); Selangor: Petaling, 5 Jul 1889, Ridley, H.N. s.n. (SING); Kanching Reserve, 17 Sep 1925, Strugnell, E.J. 10515 (K); Kanching River, 250 m, 3 Oct 1974, Van Balgooy, M.M.J. 2118 (E, L, NY); Templer National Park, Bukit Perangin, 1110 m, Cultivated C7650 (E); Gombak, Bukit Lagong FR, Sg. Kepong, 392 m, 6 Sep 2007, Chan, Y.M. FRI49298 (KEP); Bukit Lagong Forest Reserve, 300 m, 24 Sep 1980, Kochummen, K.M. FRI29126 (K, KEP, L, SING); Genting Highlands, Gombak F.R., 580 m, 23 Nov 1939, Wong, Y.K. KEP93272 (K); Ridge above Gombak Road, 4 Oct 1970, Stone, B.C.M. 9566 (KLU); Sungei Buloh, Aug 1908, Ridley, H.N. 13372 (BM, SING); ibidem, 80 m, 4 Dec 1960, Poore, M.E.D. 1064 (KLU); Kanching, Sg. Bangkap, 10 Oct 1974, Stone, B.C.M. \& Badaruddin 12068 (BKF, KLU); Genting Sempah ridge, 800 m, 21 May 1982, Stone, B.C.M. 15167 (KLU); ibidem, 760 m, 6 Mar 1973, Stone, B.C.M. 11091 (KLU); ibidem, 850 m, 18 Aug 1974, Stone, B.C.M. s.n. (KLU); Terengganu: Ulu Sungei Setiu, 28 Apr 1986, Kiew, R. RK2262 (KEP); Hulu Terengganu, Tembat Forest Reserve, 364 m, 1 Apr 2009, Mohd. Hairul, M.A. FRI60925 (KEP, SING); Besut, Sungai Kemia, 210 m, 29 Aug 1996, Chua, L.S.L. FRI26700 (KEP).

Notes. This is one of the most readily identified species of Aeschynanthus in Peninsular Malaysia due to its wide cup-shaped red calyx. It is most similar to Aeschynanthus 
tricolor Hook. from Borneo but that species mostly has a shorter corolla and has dark lines extending down the corolla tube (absent in A. obconicus). It is also similar to Aeschynanthus wallichii but that species has a smaller, more open and green calyx.

9. Aeschynanthus pulcher (Blume) G.Don, Gen. Syst. 4: 656 (1838); A.DC., Prod. 9: 262 (1845); Zollinger, Syst. Verz. 3: 56 (1855); Miquel, Fl. Ned. Ind. 2: 721 (1858); C.B.Clarke in A.DC. \& C.DC., Monogr. Phan. 5(1): 43 (1883); Middleton, Edinburgh J. Bot. 64: 412 (2007); Middleton, Edinburgh J. Bot. 66: 437 (2009). - Trichosporum pulchrum Blume, Bijdr. Fl. Ned. Ind. 764 (1826). - TYPE: Indonesia, Java, Blume, C.L. s.n. (lectotype L [Leiden number 903,307-167], designated by Middleton (2007)). (Fig. 12, 13)

Aeschynanthus parvifolius R.Br., Cyrtandreae 115 (1839); Brown in Bennett, Pl. Jav. Rar. 115 (1840); A.DC., Prod. 9: 262 (1845); Miquel, Fl. Ned. Ind. 2: 720 (1858); C.B.Clarke in A.DC. \& C.DC., Monogr. Phan. 5(1): 42 (1883); Ridley, Fl. Mal. Pen. 2: 500 (1923); Henderson, Malay. Wild. Fl. Dicot. 342 (1959); Chin, Gard. Bull. Singapore 32: 147 (1979); Stone, Fed. Mus. J. 26 (1): 98 (1981); Turner, Gard. Bull. Singapore 45: 92 (1993); Turner, Gard. Bull. Singapore 47: 243 (1997 ['1995’]); Smitinand, Thai Pl. Names ed. 2, 15 (2001). - Trichosporum parvifolium (R.Br.) Kuntze, Revis. Gen. Pl. 478 (1891). - TYPE: Indonesia, Sumatra, Pulau Bangka, 1813, Horsfield, T. s.n. (lectotype BM [BM000847144], designated by Middleton (2007)).

Aeschynanthus boschianus de Vriese, Ann. Soc. Agric. Bot. Gand 1: 403 (1845); Van Houtte, Hort. Vanhoutt. 1 (2): 31 (1846). - Trichosporum boschianum (de Vriese) Kuntze, Revis. Gen. Pl. 477 (1891); Merrill, Contr. Arnold Arbor. 8: 151 (1934). TYPE: Cultivated plant grown by Jacob-Makoy in Belgium from plant collected on Mt Gede in Java (untraced).

Aeschynanthus lobbianus Hook., Bot. Mag. 72: t.4260 (1846); Miquel, Fl. Ned. Ind. 2: 721 (1858); C.B.Clarke in A.DC. \& C.DC., Monogr. Phan. 5(1): 44 (1883); Clarke in Hooker, Fl. Brit. Ind. 4: 343 (1884); Ridley, J. Linn. Soc. Bot. 32: 501 (1896); Ridley, J. Straits Branch Roy. Asiat. Soc. 44: 16 (1905); Ridley, J. Asiat. Soc. Bengal 74 (2): 735 (1909). - Trichosporum lobbianum (Hook.) Kuntze, Revis. Gen. Pl. 478 (1891). TYPE: Indonesia, Java, Lobb, T. s.n. (holotype K).

Aeschynanthus javanicus Rollinson ex Hook., Bot. Mag. 76: t.4503 (1850); Miquel, Fl. Ned. Ind. 2: 721 (1858); C.B.Clarke in A.DC. \& C.DC., Monogr. Phan. 5(1): 44 (1883). - Trichosporum javanicum (Hook.) Kuntze, Revis. Gen. Pl. 477 (1891). TYPE: Indonesia, Java, unknown s.n. (holotype K).

Aeschynanthus neesii Zoll. \& Moritzi in H.Zollinger, Syst. Verz. 3: 56 (1855), nom. nud. - Based on Zollinger, H. 1546 (P). 


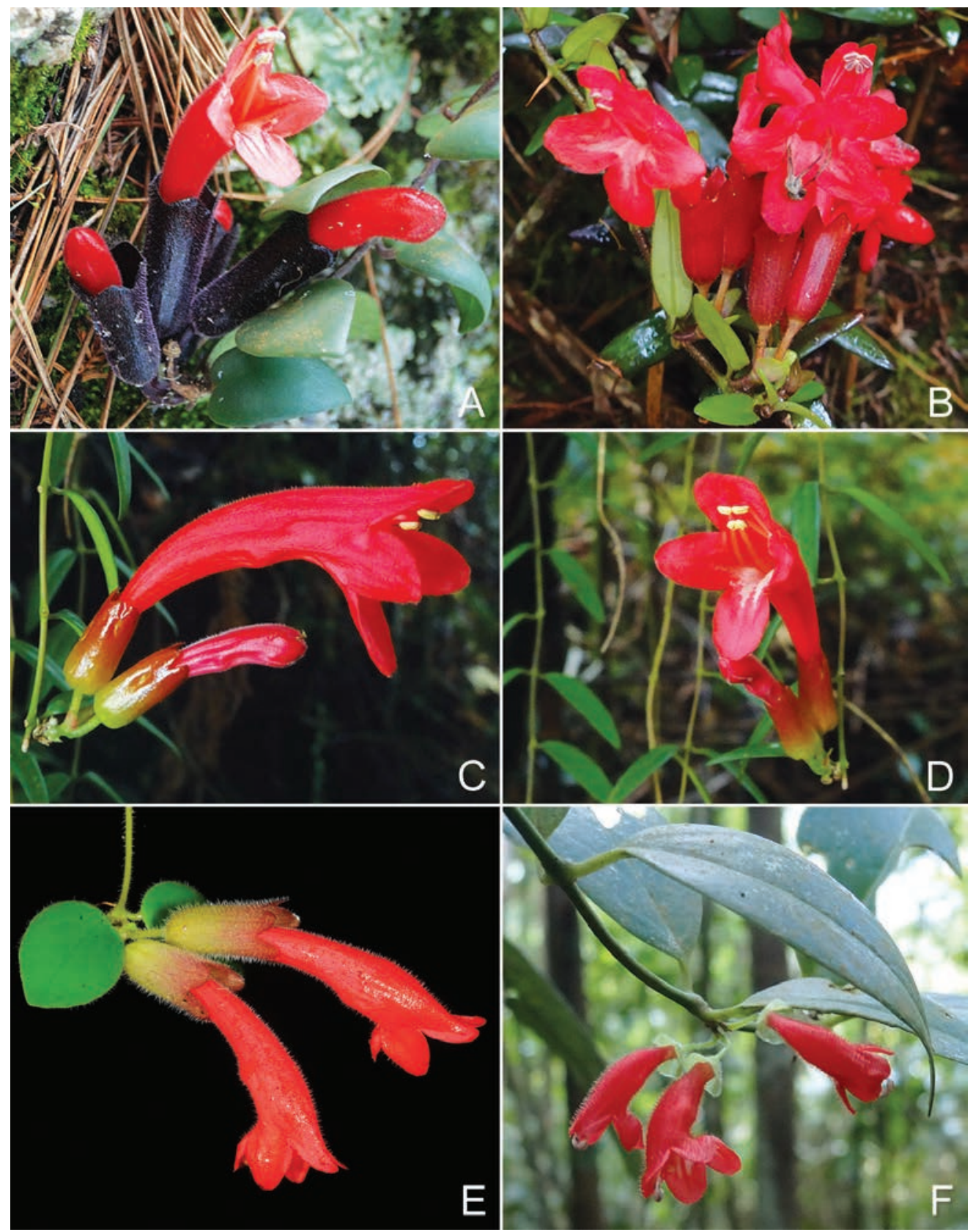

Fig. 12. A-D. Aeschynanthus pulcher (Blume) G.Don showing the variation in flower morphology and colour, particularly in the calyx. E. Aeschynanthus radicans Jack. F. Aeschynanthus wallichii R.Br. (Photos: A, David Middleton; B-D, Jana Leong-Škorničková; E, Preecha Karaket; F, Ali Ibrahim) 


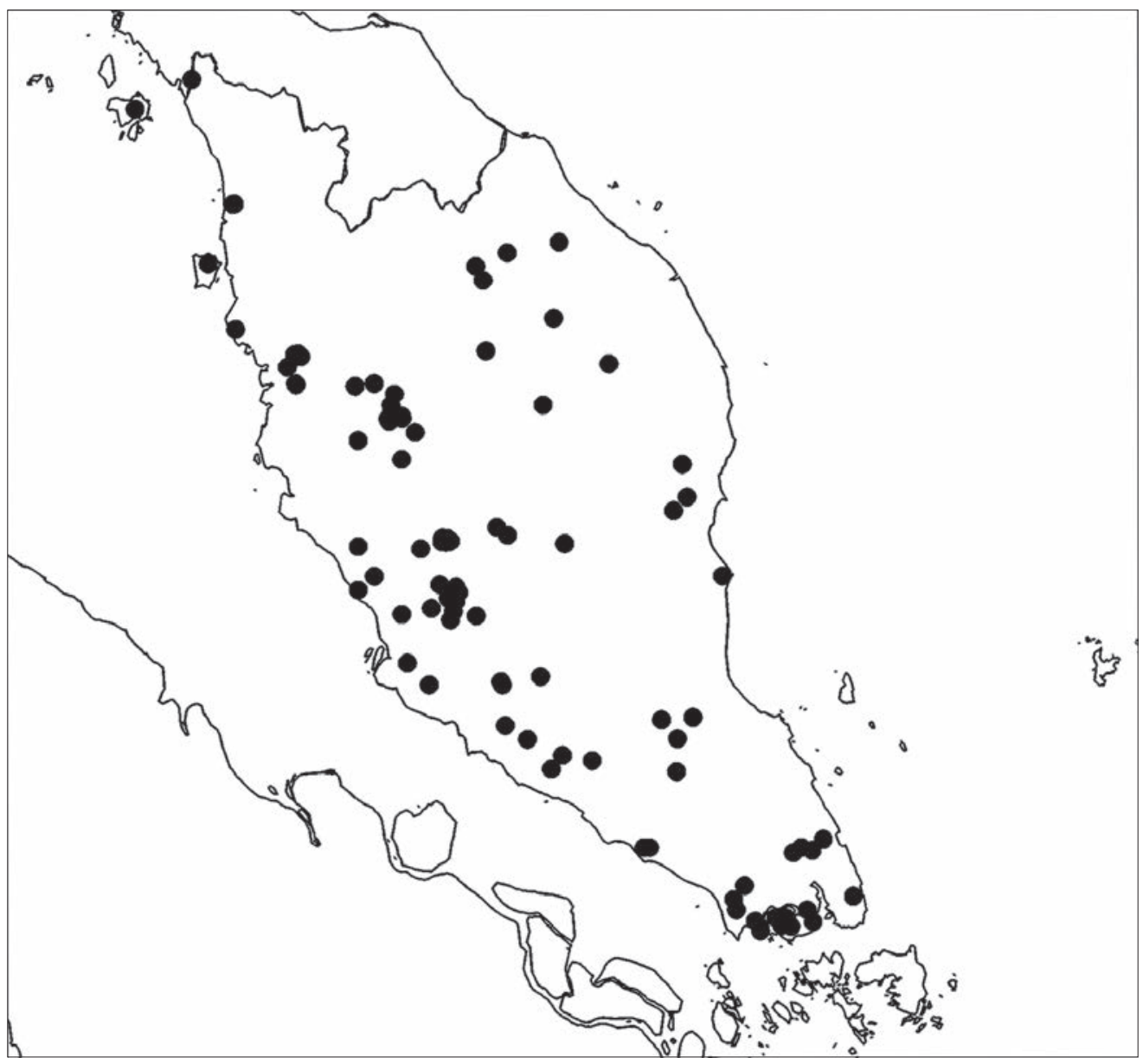

Fig. 13. Distribution of Aeschynanthus pulcher (Blume) G.Don in Singapore and Peninsular Malaysia (•).

Aeschynanthus lampongus Miq., Fl. Ned. Ind., Eerste Bijv. 563 (1861); C.B.Clarke in A.DC. \& C.DC., Monogr. Phan. 5(1): 42 (1883). - Trichosporum lampongum (Miq.) Burkill, Kew Bull. 1935: 319 (1935). - TYPE: Indonesia, Sumatra, Lampung, Radjabassa, 910 m, Teijsmann, J.E. HB4482 (lectotype U, designated by Middleton (2009); isolectotype L).

Aeschynanthus beccarii C.B.Clarke in A.DC. \& C.DC., Monogr. Phan. 5(1): 47 (1883). - Trichosporum beccarii (C.B.Clarke) Kuntze, Revis. Gen. Pl. 477 (1891). - TYPE: Indonesia, Sumatra, Sumatera Barat, Padang, Beccari, O. 796 (lectotype K, designated by Middleton (2009); isolectotypes BM [BM000537111], FI n.v., K, MEL).

Aeschynanthus zollingeri C.B.Clarke in A.DC. \& C.DC., Monogr. Phan. 5(1): 44 (1883). - Trichosporum zollingeri (C.B.Clarke) Kuntze, Revis. Gen. Pl. 478 (1891). - 
TYPE: Indonesia, Java, Zollinger, H. 1512 (lectotype BM [BM001125703], designated by Middleton (2009); isolectotypes B [presumably destroyed], P [P00492368]).

Aeschynanthus lanceolatus Ridl., J. Bot. 62: 299 (1924); Ridley, Fl. Malay Penin. 5: 324 (1925); Turner, Gard. Bull. Singapore 47: 243 (1997 ['1995’]). - TYPE: Peninsular Malaysia, Pahang, Fraser’s Hill, 1210-1320 m, 16 October 1922, Burkill, H.M. \& Holttum, R.E. 8418 (holotype K).

Aeschynanthus lampongus var. parvifolius Ridl., Fl. Malay Penin. 5: 324 (1925); Turner, Gard. Bull. Singapore 47: 243 (1997 ['1995’]). - TYPE: Peninsular Malaysia, Pahang, Puku, Kuala Teku, 20 December 1920, Seimund, E. s.n. (lectotype K, designated by Middleton (2007); isolectotype SING).

Lithophytic or epiphytic, hanging or creeping, stems dark purple to green, sparsely puberulent to glabrous. Leaves opposite; petiole 1-3(6) mm long, sparsely puberulent or glabrous; blade coriaceous or slightly fleshy, elliptic or ovate, dark green to purplish above and beneath, not variegated, 0.9-5.9 × 0.25-2.9 cm, 1.3-5.6 times as long as wide, apex rounded to acuminate, base subcordate to cuneate, glabrous above, glabrous to very sparsely puberulent all over beneath, margin entire, secondary veins weakly visible or obscure, c. 3 pairs, tertiary venation obscure. Inflorescences subterminal or axillary, 1-2-flowered; peduncle 0-12.5 mm long; bracts elliptic or ovate, 3-6.5 × 2-5.5 mm; pedicels 6-13 mm long, green, sparsely puberulent to densely long hairy. Calyx with a tube for most of length and free lobes, dark red to purplish or purplish brown, mostly sparsely eglandular puberulent, more rarely densely puberulent, glabrous or with a few hairs only on very tips, 11.5-29 mm long; tube 10-25 mm long, 89-96\% of total length, 6-21 mm wide at top of tube; lobes triangular, semicircular or merely a weak curve of the upper rim, slightly spreading or erect, 1-5 × 4-7 mm, apex rounded or obtuse. Corolla 42-66 mm long, inflated at base, externally tube dark or bright red, often white at extreme base inside calyx tube, lobes bright red, internally lobes bright red with cream and dark markings on lower 3 lobes; upper lobes oblong or ovate, spreading or not, 5.7-13 × 1.9-6.5 mm, sinus 2.2-4.6 mm deep, apex rounded; lateral lobes deltoid or ovate, spreading or not, 6.1-11.5 × 6.5-10.5 $\mathrm{mm}$, apex rounded; lower lobe elliptic or ovate, spreading or not, 7.6-13 $\times 6.5-8 \mathrm{~mm}$, apex rounded; outside sparsely to densely puberulent, inside glabrous or with scattered papillae or small glandular hairs which are denser in the upper half, sessile glands below lobes. Stamens reaching end of corolla or slightly exserted, fused in 2 pairs; filaments red, glabrous or with very few sessile glands or papillose; anterior filaments inserted at $29-47 \mathrm{~mm}$ from corolla base which is $54-68 \%$ of corolla length, filaments 21-27 mm long, anthers 2.4-3.3 × 0.8-1.6 mm; posterior filaments inserted at 33.5$41 \mathrm{~mm}$ from corolla base which is $60-70 \%$ of corolla length, filaments $17.5-21 \mathrm{~mm}$ long, anthers 2.3-2.9 × 0.8-1.9 mm; staminode 0.5-1.6 mm long. Disk 0.8-1.5 mm high, strongly 5-lobed, 5-crenate or a simple annular ring. Pistil 37-58 mm long; stipe 16-27 mm long, puberulent; ovary pale yellow, 14-30 mm long, minutely papillose or with sessile glands; style pale yellow, 4.5-14 mm long, densely pubescent. Capsule 
18-40 cm long, 2-4 mm wide. Seed grain 0.6-0.9 × 0.2-0.3 mm, papillose, bubble cells present at base of hilar appendage; apical appendage a filiform hair, 7-9.5 mm long; hilar appendage a single filiform hair, 6-9 mm long; appendages not papillose.

Distribution. Extreme south of Thailand, southern Vietnam, Peninsular Malaysia (Johor, Kedah, Kelantan, Melaka, Negeri Sembilan, Pahang, Penang, Perak, Perlis, Selangor, Terengganu), Singapore, Sumatra, Java, ?Borneo. See also note below.

Habitat and ecology. Epiphytic or clambering over rocks, most common in lower montane forest, sometimes in lowland, hill dipterocarp or upper montane forest, in peat swamp forest or in forest on quartzite or sandstone, at 0-2100 m altitude.

Provisional IUCN conservation assessment. Least Concern (LC). This species is widespread and locally common. In Singapore it was long thought to be nationally extinct but has been rediscovered in Nee Soon Swamp (Williams, 2014).

Additional Singaporean and Peninsular Malaysian specimens examined. PENINSULAR MALAYSIA: unknown loc., Jan 1882, Kunstler, H. 2636 (NY); Johor: unknown loc., May 1924, Franck, C.W. 209 (C); ibidem, 6 Jun 1965, Jumali K2077 (SINU); Pontian, Pengkalan Raja, 25 Jun 1939, Corner, E.J.H. \& Henderson, M.R. SFN36611 (K, P), SFN36612 (SING); western tip of Gunong [Gunung] Panti West, 560 m, 18 Jul 1981, Maxwell, J.F. 81-166 (AAU, KLU, SING); Gunong [Gunung] Panti, 1 Aug 1960, unknown s.n. (SINU); ibidem, 480 m, 4 Feb 1981, Collenette, I.S. 2253 (E); ibidem, 520 m, 5 Jul 1970, Samsuri Ahmad S.311 (BKF, C, G, K, KLU, L [2 sheets], LAE, SING); ibidem, 460 m, 31 Jul 2008, Yao, T.L., Lim, C.L., Rosdi, M. \& Ayau, K. FRI65387 (KEP); Kuala Sedili Road, 26 May 1961, Burkill, H.M. HMB2665 (K, SING); Kota Tinggi, Kuala Sedili New Road, 23 Jun 1959, Kadim \& Noor, M. 135 (E, K, L, LAE, SING); Mawai-Sedili Road, 8 Feb 1961, Chew, W.-L. CWL224 (C, E, K, L, SING); 8th mile Mawai-Kuala Sedili New Road, 8 Feb 1961, Sinclair, J. 10568 (E, L, SING); Mersing, Lombong Batu, 6 Jun 1965, Keng, H. \& Jumali K2077 (L); Kulai FR, Gunung Pulai, 29 Jun 1987, Zainudin, A. et al. AZ2438 (UKMB); Penggaram, Nov 1900, Ridley, H.N. s.n. (SING); Batu Pahat, 1892, Nongchi s.n. (SING); Tanjong Kupang, 1894, Ridley, H.N. s.n. (SING); Tanah Runto, 14 Feb 1890, Goodenough, J.S. s.n. (SING); ibidem, 22 Apr 1890, Ridley, H.N. s.n. (SING); Batu Pahat, Nov 1900, Ridley, H.N. s.n. (SING); Tempayang River, Apr 1909, Ridley, H.N. s.n. (SING); Kulai Hutan Simpan, Gunung Pulai, 24 Apr 1922, Md Nur \& Kiah 7783 (SING); Jambu Larang, Oct 1892, Fielding, J. s.n. (SING); Sungei Tukong, 0 m, 20 Dec 1931, Spare, G.H. 991 (SING); Segamat, Gunung Chabang Tiga, Gunung Chabang Tiga South Peak, 935 m, 25 Aug 2007, Chew, M.Y. \& Rosdi, M. FRI55583 (KEP); Gunong [Gunung] Bekok, 18 Aug 1968, Jumali \& Heaslett, E.A. KJ732 (SINU); Gunung Ledang, 7 Dec 2010, Yeo, C.K. s.n. (SINU); ibidem, 24 Feb 1980, Zainal Mustafa ZM64 (UKMB); ibidem, 26 Apr 1972, Year III students FSC312 (KLU); Gunung Ledang, Above Bukit Besar, 12 Jun 1892, Ridley, H.N. s.n. (SING); Kedah: unknown loc., 24 Apr 1868, Maingay, A.C. K.D.1218 (K); Langkawi, Gunong [Gunung] Raya, Sep 1890, Curtis, C. 2503 (SING); Kedah Peak, 1060 m, Dec 1915, Robinson, H.C. \& Kloss, C.B. 5997 (K, SING); ibidem, 910 m, 2 Dec 1915, Robinson, H.C. \& Kloss, C.B. 6049 (K, SING); ibidem, 1000-1400 m, 21 Jan 1983, Davis 69431 (E); ibidem, 1893, Ridley, H.N. s.n. (SING); ibidem, 910-1210 m, 3 Jun 1971, Keng, H. et al. 67 (SING, SINU); ibidem, Aug 1893, Ridley, H.N. 5513 (BM, SING); Gunung Jerai, 850 m, 12 Feb 1961, Yong KEP99330 (K, KEP); ibidem, 1200 m, 14 Sep 1979, Rao \& et al. 167 
(L); ibidem, 8 Nov 1962, Samsuri Ahmad 309 (SING); ibidem, 1121 m, 9 Feb 2010, Imin, K., Nor Ezzawanis, A.T., Hovenkamp, P. \& Angan, A. FRI66493 (KEP); ibidem, 3 Sep 1995, Choo, J.P.S., Chua, D.W.S., Lian, S.H.L., Lim, P.Y. \& Wong, J.Y. PYL2 (SINU); ibidem, 1200 m, 14 Sep 1979, Rao \& et al. 167 (SINU); ibidem, 850 m, 15 May 1969, Smith, G. 513 (KLU); ibidem, 1060 m, 10 Jul 1977, Lo, Y.N. \& Mahmud 83 (KLU); ibidem, 1150 m, 13 May 1969, Stone, B.C.M. \& Mahmud 8500 (KLU); Kelantan: S. Lebir, Sungai Terang, 8 Jul 1935, Henderson, M.R. SFN29643 (K, SING); Gua Musang, 3 Aug 1962, UNESCO limestone expedition 239 (SING); Gua Musang, Gua Batu Boh, 300 m, 12 Aug 1971, Chin, S.C. 1441 (L); Kuala Krai, Gunung Stong Utara Forest Reserve, VJR Gunung Stong Utara, 180 m, 19 Jun 2001, Sam, Y.-Y. FRI46602 (KEP); Dabong, Gunung Setong, 15 May 1988, Kiew, R. RK2727 (SING); Melaka: unknown loc., 7 Oct 1865, Maingay, A.C. K.D.1219 (K); Ayer Panas, Nov 1893, Ridley, H.N. 1578 (BM, SING); Jus, Oct 1893, Goodenough, J.S. 1711 (SING); Jasin, Bkt. Senggeh FR, Batu Lebah, Kg. Bkt. Senggeh, 175 m, 28 Aug 2007, Chan, K.Y. FRI49293 (KEP); Negeri Sembilan: Serting Ulu Forest Reserve, 4 Jun 1992, Saw, L.G. \& Mustafa, D. FRI37514 (K, KEP); Jelebu, Gunung Telapa Buruk, 1000 m, 21 Apr 1988, Kiew, R. RK2641 (KEP); Rembau, Gunung Datuk, 400 m, 10 Jul 1979, Asmah 22 (UKMB); Tampin, Tampin Forest Reserve, Hutan Lipur G. Tampin, 351 m, 5 May 2009, Chan, K.Y. FRI64737 (KEP, SING); Jelebu, Bkt Tangga, G. Telapak Burok, 1038 m, 11 Apr 2008, Rosdi, M. \& Rafidah, A.R. FRI59837 (KEP); Pahang: Genting Highlands, 1150 m, 11 Jun 1978, Stone, B.C.M. 13764 (KLU); ibidem, 25 Nov 1984, Kiew, R. RK1548 (KEP); ibidem, 1450 m, 14 Mar 1982, Stone, B.C.M. 15134 (KLU); ibidem, 15 Nov 1966, Stone, B.C.M. 6543 (G, KLU, L); ibidem, 910 m, 9 May 1972, Kochummen, K.M. FRI16516 (KEP); Genting Highlands, Gunung Ulu Kali, 1600 m, 9 Apr 1978, Maxwell, J.F. 78-90 (L); Genting Sempah ridge, Nov 1970, Stone, B.C.M. s.n. (KLU); Kuantan, Bukit Berserah Forest Reserve, 3 Sep 1933, Mahmood KEP17216 (K, KEP, L); Kwantan [Kuantan], Sep 1889, Durnford, L. s.n. (SING); Pekan, Jul 1917, Evans, J.H.N. s.n. (K); ibidem, 1 Apr 1890, Haviland, G.D. s.n. (SING); ibidem, 30 Nov 1924, Burkill, H.M. \& Md Haniff 17258 (SING); Bukit Cheras, 150 m, 11 Oct 1931, Henderson, M.R. 25065 (SING); Rompin, 19 May 1919, Gerb 3257 (SING); ibidem, 15 Nov 1929, Mahammud 17178 (SING); Gunung Mengkuang, 29 Mar 1959, unknown KEP78844 (KEP); Temerluh, Krau GR, Trail to Batu Bergambar from Batu Gajah, 550 m, 4 Jun 2000, Chua, L.S.L., Damahuri, S., Ayau, K. \& Ramli, P. FRI45633 (KEP); Gunung Senyum, 29 Jul 1929, Henderson, M.R. s.n. (SING); Fraser’s Hill, 1210 m, 18 Apr 1955, Purseglove, J.W. P4182 (K, L, LAE, SING); ibidem, 4 Oct 1961, Burkill, H.M. HMB2811 (K, L, LAE, SING); ibidem, 1300 m, 19 Apr 1955, Purseglove, J.W. P4202 (E, K, L, SING); ibidem, 1210 m, 24 Sep 1959, Shah, M. \& Noor, M. MS607 (E, K, SING); ibidem, 16 Jul 2002, Bramley, G. \& Sam, Y.-Y. GB27 (E, KEP); ibidem, 450 m, 16 Apr 1962, Burtt, B.L. \& Woods, P.J.B. B1643 (E); ibidem, 22 Dec 1979, Bremer, B. \& Bremer, K. 1808 (KLU, L); ibidem, 1240-1270 m, 3 Oct 1987, Worthington, R.D. 13326 (L); ibidem, 16 Dec 1979, Kiew, R. RK832 (KEP); ibidem, 21 Nov 1980, Kiew, $R$. RK1002 (KEP); ibidem, 13 Feb 1986, Kiew, R. RK2160 (KEP); ibidem, 13 Mar 1986, Anthony, S. SA482 (KEP); ibidem, 10 Nov 1987, Anthony, S. SA897 (KEP); ibidem, 1030 m, Kassim, M. 550 (UKMB); ibidem, 25 Oct 1979, Zainudin, A. AZ40 (UKMB); ibidem, 31 Oct 1991, Kiew, R. RK3356 (SING); ibidem, 1210 m, 13 Jul 1957, Hawkins, A.S.M. s.n. (SING); ibidem, 1210 m, 25 Aug 1923, Henderson, M.R. FMS11263 (SING); ibidem, 14 Jun 1930, Kalong 22423 (SING); ibidem, 13 Nov 1981, Keng, H. et al. 86 (SING); ibidem, 21 Jun 2006, Phoon, S.N., Kamarudin S., Kueh, H.L. \& Rafidah, A.R. FRI51576 (KEP); ibidem, Mendum, M. s.n. (E); ibidem, 26 Aug 1991, Kiew, R. RK3264 (SING); ibidem, 1240 m, 25 Aug 1959, Burkill, H.M. HMB1996 (SING); ibidem, 1210-1320 m, 16-30 Sep 1922, Burkill, H.M. \& Holttum, R.E. s.n. (SING); ibidem, 21 Nov 1977, Keng, H. et al. 97 (SINU); ibidem, 5 Nov 1973, Keng, H. et al. 
54 (SINU); ibidem, 28 Oct 1974, Keng, H. et al. 93 (SINU); ibidem, 13 Nov 1981, Keng, H. et al. CTV86 (SINU); ibidem, Dec 1970, Mahmud bin Sidek s.n. (KLU); ibidem, Sep 1940, Addison, G.H. s.n. (SING); ibidem, 1120 m, 29 Aug 1923, Md Nur 11138 (SING); ibidem, 1210 m, 29 Aug 1923, Henderson, M.R. FMS11453 (SING); Fraser’s Hill, Jeriau Road, 1060-1210 m, 20 Aug 1960, Burkill, H.M., Shah, M. \& Noor, M. HMB2430 (SING); ibidem, 1090 m, 17 Jun 1972, Stone, B.C.M. 10798 (KLU); Fraser's Hill, Mager Trail, 1390 m, 3 May 2007, Chew, M.Y., Imin, K., Kiew, R. \& Nooteboom, H.P. FRI53648 (E, KEP, SING); Fraser's Hill, Jalan High Pines, 1200 m, 13 Nov 2006, Phoon, S.N. FRI51987 (KEP, KLU, SING); Fraser's Hill, Pine tree hill, 1450 m, 19 Sep 1922, Burkill, H.M. \& Holttum, R.E. 8549 (SING); Fraser's Hill, Richmond, 27 Oct 2010, Imin, K., Utteridge, T.M.A. \& Julius, A. FRI71855 (KEP); Fraser's Hill, Nuri Trail, 3 Dec 1991, Kiew, R. \& Anthony, S. RK3414 (KEP); Fraser's Hill, Gunong [Gunung] Peninjau, 1300 m, 26 Aug 1959, Burkill, H.M. HMB2038 (K, SING); Cameron Highlands, 1450 m, 1 Apr 1930, Henderson, M.R. s.n. (NY, SING); ibidem, 1976, Anthony, S. SA233 (KEP); ibidem, 1450 m, 18 Jan 1924, Henderson, M.R. 11713 (SING); ibidem, 10 Jul 1979, Everard, B. \& Young, D. 94 (K); Cameron Highlands, Tanah Rata, 1450 m, 23 Nov 1925, Henderson, M.R. SFN17921 (K, SING); Cameron Highlands, Sungai Palas Estate, 1520 m, 8 Sep 1956, Burkill, H.M. HMB856 (K, L, SING); Cameron Highlands, Sungai Ichat, 19 Feb 2008, Rafidah, A.R., Imin, K. \& Ummul Nazrah, A.R. FRI55631 (KEP, SING); Cameron Highlands, 1 km to Sg. Bisik Waterfall from Ringglet road, 1201 m, 19 Nov 2009, Imin, K., Ong, P.T., Kueh, H.L. \& Paiman, N. FRI66402 (KEP); Rompin, Endau-Rompin State Park, Trail to Gunung Keriong, 260 m, 20 Aug 2002, Sam, Y.-Y., Apok, K. \& Markandan, M. FRI47150 (KEP); Cameron Highlands, Sungai Ichat, 12 Aug 1931, Jaemat 25181 (KEP); Cameron Highlands, G. Siku FR, Trail to G. Siku, 1545 m, 21 May 2007, Rosdi, M., Imin, K. \& Ummul Nazrah, A.R. et al. FRI58753 (KEP); Cameron Highlands, Gunung Siku Forest Reserve, 1535 m, 25 Oct 2007, Imin, K. et al. FRI58557 (KEP, SING); Cameron Highlands, Path to Taman Sedia, 6 Apr 1934, Symington, C.F. 36073 (KEP); Cameron Highlands, Jln Kamunting 44th mile, 1520 m, 21 Jul 1965, Khoo, R. \& Ng, S.M. 074 (KLU); Penang: Government Hill, Jul 1894, Curtis, C. s.n. (SING); Perak: unknown loc., Scortechini, B. 36a (SING); Ulu Kinta, 620 m, 19 Apr 1961, Castle-Smith, P.M. 15 (K); Gunung Korbu, 610 m, 24 Mar 1913, Robinson, H.C. s.n. (K); Waterfall north of Tana Rata acc. no. 19680649 vouchered as , Cultivated C7431 (E); Kuala Kangsar, Bubu FR, Gunung Bubu, 938 m, 10 Mar 2010, Julius, A., Coode, M.J.E. \& Angan, A. FRI57716 (KEP); Kuala Kangsar, 850 m, 8 Apr 1995, Chua, L.S.L. FRI39082 (KEP); Sunga Ryah, Scortechini, B. 39 (SING); Gunung Batu Puteh, Wray, L. 873 (SING); Bujang Melaka, 1898, Ridley, H.N. s.n. (SING); Taiping, 910 m, 12 Feb 1917, Md Haniff \& Md Nur 2314 (K, SING); ibidem, Mar 1911, Anderson, J.W. 156 (SING); ibidem, Dec 1902, Ridley, H.N. s.n. (SING); ibidem, Nov 1952, Carter s.n. (SING); Taiping, Gunung Hijau, 14 Jul 1906, Ernst, A. 1189 (L); ibidem, 8 Oct 1899, Fox, W. s.n. (SING); Taiping, Bukit Larut, Oct 1908, Long, F.R. 14 (K); ibidem, 24 Nov 1999, Kiew, R. RK4869 (SING); ibidem, 9 Mar 1939, Spare, G.H. 2139 (SING); ibidem, 1130-1240 m, 9 Sep 1949, Sinclair, J. SFN38609 (BM, E, K, L, SING); ibidem, Wray, L. 655 (K, SING); ibidem, 20 Oct 1992, Kamarudin S. FRI34629 (K); ibidem, Birch Hill, 1330 m, 9 Sep 1977, Lewis, G.P. 251 (K, KEP); ibidem, 1060-1210 m, 3 Dec 1965, Shah, M. \& Sidek MS1064 (E, L, SING); ibidem, Sep, Wray, L. 3217 (SING); ibidem, 1210 m, 22 Nov 1980, Keng, H. et al. 27 (SINU); ibidem, Aug 1967, unknown K6502 (SINU); ibidem, 4 Sep 1995, Heng, H.P. et al. HP12 (SINU); ibidem, 1090 m, 2 Mar 1924, Burkill, H.M. \& Md Haniff 12971 (SING); ibidem, 28 Feb 1924, Burkill, H.M. \& Md Haniff 12833 (SING); ibidem, 1210-1360 m, 6 Mar 1939, Spare, G.H. 2018 (SING); ibidem, Mar 1884, Scortechini, B. 291 (SING); ibidem, 1000 m, 29 Oct 1969, Everett, B. FRI13553 (KEP); ibidem, 16 Jan 1994, Anthonysamy, S. SA1155 (KEP); ibidem, 880-1060 m, 15 May 1992, 
Rahimatsah Amat N12 (KEP); ibidem, 1060 m, 23 Oct 1962, Merton, L.F.H. 004194 (KLU); ibidem, 1080 m, 20 Mar 2007, Julius, A. FRI54942 (KEP, SING); ibidem, Sep 1889, Curtis, C. s.n. (SING); ibidem, 25 Oct 1972, Hons. Students 1 (SING); ibidem, 910 m, 29 Oct 1968, Smith, G. 448 (KLU); Perlis: Bukit Bintang Forest Reserve, 7 May 1988, Latiff, A. \& Zainudin, A. ALM2764 (UKMB); Selangor: Klang, Bukit Changgang, 1 Oct 1937, Md Nur SFN33963 (SING); Klang Gates Rridge, 4 Oct 1981, Kiew, R. RK1081 (KEP); Chemara, 3 Jan 1959, Carrick, J. 607 (KLU); Telok Forest Reserve, 21 Feb 1969, Kochummen, K.M. FRI2654 (KEP); ibidem, 18 Jul 1963, unknown 4636 (KLU); Sungei Buloh, 30 Nov 1889, Goodenough, J.S. s.n. (SING); ibidem, 1890, Goodenough, J.S. s.n. (SING); Gunong [Gunung] Bunga Bua, 28 May 1966, Ng, F.S.P. FRI1136 (KEP, SING); Semangkok Pass, Feb 1904, Napier, W. s.n. (SING); Tanjong Karang, Sungei Tinggi, 30 m, 27 Sep 1975, Samsuri Ahmad SA1125 (SING); Hulu Langat, Gn. Nuang, Above Pacat Camp, 1416 m, 12 Nov 2013, Yao, T.L., Imin, K., Mohd Nazri, A. \& Kueh, H.L. FRI77328 (KEP); Gombak, Klang Gates Ridge, Quartz Ridge, 270 m, 6 Apr 2007, Syahida-Emiza \& Angan, A. FRI55108 (KEP); Kanching, Bukit Takun, 300-400 m, 19 Mar 1972, Chin, S.C. 1812 (KLU); ibidem, 300 m, 11 Jul 1965, Stone, B.C.M. 5889 (KLU); Ulu Besut, Bukit Tangga, 610 m, 20 Jul 1984, Shah, M. \& Mahmud MS4978 (E, SING); Kuala Selangor, Sg. Karang FR, 26 m, 1 Jul 2013, Lim, C.L. FRI73033 (KEP); Kuala Selangor, Sungai Tinggi, 14 Oct 1937, Md Nur SFN34079 (BM, K, L, MICH, P, SING); Kanching, Bukit Takun, 150 m, 3 Nov 1937, Md Nur SFN34400 (K, MICH, SING, US); Telok Forest Reserve, Mar 1965, Whitmore, T.C. FRI227 (K, KEP, L, SING); Genting Highlands, Gunung Bunga Buah, 10 May 1991, Tan, W.K. et al. TWK12 (SING); Genting Highlands, Old trail to Gunung Bunga Buah, 1095 m, 18 Jan 2008, Chan, Y.M. \& Kiew, R. et al. FRI60504 (KEP); Genting Highlands, Ulu Gombak, 910 m, 14 Jun 1973, Mohd Shah \& Mohd Ali MS 3046 (C, SING); Terengganu: Ulu Besut, Bukit Tangga, 910 m, 18 Jul 1984, Shah, M. \& Mahmud MS4895 (E, KEP, SING); Bundi, Feb 1904, Rostado 64 (SING); Hulu Terengganu, Sg. Cicir, 4 Aug 2007, Jutta, M. \& Kueh, H.L. FRI59583 (KEP).

SINGAPORE: Changi, 10 Dec 1889, Ridley, H.N. 2706 (K); MacRitchie Reservoir, 4 Dec 1948, Sinclair, J. 5376 (E); Bukit Timah, 18 May 1964, Hardial, S. \& Samsuri Ahmad 18 (C, L, LAE, SING); Krangi, 30 Nov 1899, Ridley, H.N. s.n. (L); Krangi, 1891, Goodenough, J.S. 2705 (SING); Krangi, 29 Nov 1889, Goodenough, J.S. 2706 (BM, SING); Tuas, 1 May 1891, Goodenough, J.S. s.n. (SING); Chan Chu Kang, May 1889, Corporal s.n. (SING); Bukit Timah, 7 Jun 1974, Noor, M. MN.1913 (SING); Krangi, 10 Dec 1889, Goodenough, J.S. s.n. (SING); Tengeh Reservoir, 4 Aug 1890, Goodenough, J.S. s.n. (SING); Krangi, 2 Nov 1889, Goodenough, J.S. s.n. (SING); Krangi, 7 Dec 1889, Ridley, H.N. s.n. (SING); Krangi, 8 Apr 1890, Goodenough, J.S. s.n. (BM); Krangi, 1909, Ridley, H.N. s.n. (BM); Nee Soon Swamp Forest, 8 Aug 2010, Gwee, A.T. SING2010-443 (SING); Nee Soon Swamp Forest, 17 Feb 2009, Staples, G., Leong, P., Chew, P.T. \& Ibrahim, A. et al. SING2009-157 (KEP, SING); Nee Soon Swamp Forest, 17 Apr 2012, Leong, P., Yam, T.W., Liew, D. \& Rodda, M. et al. SING2012-165 (SING); Tengeh Reservoir, 1891, Ridley, H.N. 2710 (SING); Mandai Road, Aug 1920, Burkill, I.H. 6102 (SING); Nee Soon Swamp Forest, 8 May 2013, Ibrahim, H., Lua, H.K. \& Saiffudin, S. SING2013-093 (SING); Nee Soon Swamp Forest, 8 May 2013, Ibrahim, H. \& Lua, H.K. SING-2013-092 (SING).

Notes. This is a very widespread and variable species in both leaf and flower characters. Although it already has many synonyms further investigation is required as to whether a number of other species in Borneo should also be included in synonymy. In Peninsular Malaysia and Singapore this species has most commonly gone by the name Aeschynanthus parvifolius but was previously synonymised in Middleton (2007) as it 
falls within the range of variation of the species. Likewise, Aeschynanthus lanceolatus is not distinct from this variable species.

There are a small number of specimens with pubescent leaves, reminiscent of Aeschynanthus radicans but with the ovary pubescence of A. pulcher. Further studies should be undertaken to see if there is any possibility of hybridisation between these two similar species.

10. Aeschynanthus radicans Jack, Trans. Linn. Soc. London 14: 43 (1823); Brown, Cyrtandreae 115 (1839); Steudel, Nomencl. Bot. ed. 2, 1: 32 (1840); Brown in Bennett, Pl. Jav. Rar. 115 (1840); A.DC., Prod. 9: 262 (1845); Zollinger, Syst. Verz. $3: 56$ (1855); Miquel, Fl. Ned. Ind. 2: 720 (1858); C.B.Clarke in A.DC. \& C.DC., Monogr. Phan. 5(1): 41 (1883); Clarke in Hooker, Fl. Brit. Ind. 4: 343 (1884); Ridley, J. Linn. Soc. Bot. 32: 501 (1896); Ridley, J. Asiat. Soc. Bengal 74 (2): 736 (1909); Ridley, Fl. Mal. Pen. 2: 500 (1923); Bakhuizen van den Brink, Blumea 6: 395 (1950); Merrill, J. Arnold Arb. 33: 214 (1952); Barnett, Fl. Siam. 3 (3): 202 (1962); Backer \& Bakhuizen van den Brink, Fl. Java 2: 524 (1965); Chin, Gard. Bull. Singapore 32: 148 (1979); Turner, Gard. Bull. Singapore 45: 92 (1993); Turner, Gard. Bull. Singapore 47: 244 (1997 ['1995’]); Burtt, Thai Forest Bull., Bot. 29: 84 (2001); Smitinand, Thai Pl. Names ed. 2, 15 (2001); Middleton, Edinburgh J. Bot. 64: 414 (2007). - Trichosporum radicans (Jack) Nees, Flora 8: 144 (1825); Merrill, Contr. Arnold Arbor. 8: 152 (1934). - TYPE: Indonesia, Sumatra, Lampung, Gunung Rati Telanggaran, 400 m, 14 November 1921, Iboet 57 (neotype L, designated by Middleton (2007)). (Fig. 12, 14)

Trichosporum ovatum D.Don ex C.B.Clarke in A.DC. \& C.DC., Monogr. Phan. 5(1): 41 (1883), nom. nud.

Aeschynanthus radicans var. robustior C.B.Clarke in A.DC. \& C.DC., Monogr. Phan. 5(1): 41 (1883). - TYPE: Kalimantan, Kalimantan Selatan, Banjarmasin, Motley, J. 715 (lectotype K, designated here).

Aeschynanthus radicans var. lanuginosa Ridl., J. Asiat. Soc. Bengal 74 (2): 736 (1909); Turner, Gard. Bull. Singapore 47: 243 (1997 ['1995’]). - TYPE: Peninsular Malaysia, Perak, Scortechini, B. 330 (not traced). This variety is placed in synonymy based on the fact that the characters used to distinguish it are continuously variable in the species.

Epiphytic and hanging to lithophytic and creeping or even epiphytic and climbing by adventitious roots on the stem; stems sparsely to densely puberulent or with longer hairs. Leaves opposite; petiole 1-4.5 mm long, sparsely to densely puberulent; blade slightly fleshy, ovate, mostly green above and beneath, not marbled, orbicular or elliptic, 1-5 × 0.8-2.6 cm, 1.1-2.6 times as long as wide, apex rounded and apiculate or acute to acuminate, base subcordate to obtuse; glabrous to sparsely puberulent all over above, sparsely to densely puberulent all over beneath, margin entire; secondary 


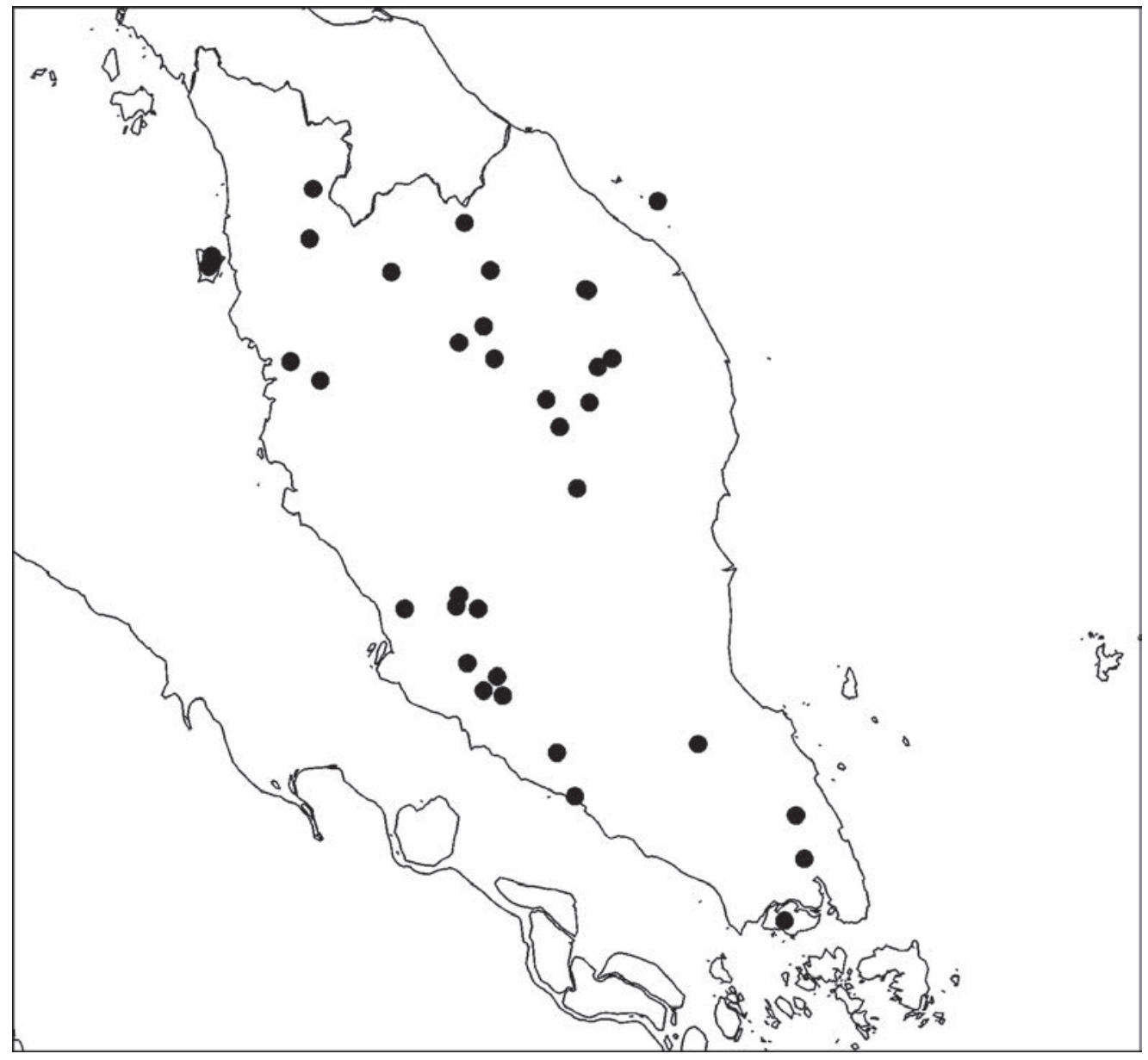

Fig. 14. Distribution of Aeschynanthus radicans Jack in Singapore and Peninsular Malaysia $(\bullet)$.

veins obscure, tertiary venation obscure. Inflorescences axillary, generally 1-flowered; peduncle 0-3 mm long, bracts elliptic to ovate, 5-6 mm long; pedicels 7-14 mm long, sparsely to densely puberulent or with longer hairs. Calyx with a tube for most of length and free lobes, rarely slightly zygomorphic, purple, green with red veins, or green, puberulent, sometimes with quite long hairs, 19.5-26 mm long; tube 13-22 mm long which is 65-91\% of total length, 6.5-9 mm wide at top of tube; lobes narrowly triangular, ovate or oblong, erect, 2-8 × 2.6-5 mm, apex rounded. Corolla 47.5-58 $\mathrm{mm}$ long, inflated at base, externally tube bright red, lobes bright red, internally tube yellowish, lobes red with yellowish at base and darker red markings on lower 3 lobes; upper lobes oblong or ovate, slightly spreading or not, 6.5-10 × 2-4.4 mm, sinus 2.4$6.7 \mathrm{~mm}$ deep, apex rounded; lateral lobes ovate or deltoid, slightly spreading or not, 7-10 × 6.2-7.5 mm, apex rounded; lower lobe elliptic or oblong, slightly spreading or not, 6.5-10.7 × 5.6-7.7 mm, apex rounded, outside densely puberulent, inside glabrous 
or with sessile glands and short stalked glandular hairs throughout, sessile glands below lobes. Stamens reaching to end of upper corolla lobes or slightly exserted, fused in 2 pairs, filaments with very few glandular hairs or sessile glands; anterior filaments inserted at $27-36 \mathrm{~mm}$ from corolla base which is $52-63 \%$ of corolla length, filaments 22-27 mm long, anthers 2.1-3.4 × 1.5-1.9 mm; posterior filaments inserted at 31-38.5 $\mathrm{mm}$ from corolla base which is $59-70 \%$ of corolla length, filaments $15.5-21 \mathrm{~mm}$ long, anthers 2.1-3 × 1.2-1.7 mm; staminode 0.5-5 mm long. Disk 1-8 mm high, a simple annular ring. Pistil 45.5-61 mm long; stipe 18-28 mm long, densely puberulent, often with a mix of glandular and eglandular hairs; ovary 14-28 mm long, densely puberulent, often with a mix of glandular and eglandular hairs; style 6-15 mm long, densely puberulent, often with a mix of glandular and eglandular hairs. Capsule 19-35 mm long, 2.5-3 mm wide. Seed grain 0.8-0.9 × 0.15-0.3 mm, papillose, bubble cells present at base of hilar appendage; apical appendage a filiform hair, 7-8 $\mathrm{mm}$ long; hilar appendage a single filiform hair, 6-8 $\mathrm{mm}$ long; appendages not papillose.

Distribution. Southern Thailand, Peninsular Malaysia (Johor, Kedah, Kelantan, Melaka, Negeri Sembilan, Pahang, Penang, Perak, Selangor, Terengganu), Singapore (but currently considered to be nationally extinct), Sumatra, Borneo.

Habitat and ecology. Epiphytic or clambering over rocks in lowland or hill dipterocarp forest, often by streams, occasionally in swamp forest, at 0-1000 m altitude.

Provisional IUCN conservation assessment. Least Concern (LC). This species is widespread and locally common although there are rather few recent collections of it from Malaysia. The last collection made of this species in Singapore was in 1931 on Bukit Timah. It has since been listed as nationally extinct by Chong et al. (2009). At one time Aeschynanthus pulcher was also listed as nationally extinct in Singapore and has since been rediscovered (Williams, 2014). Aeschynanthus radicans may also still occur in Singapore in small numbers in the forest canopy or may come back in from Peninsular Malaysia or Sumatra.

Additional Singaporean and Peninsular Malaysian specimens examined. PENINSULAR MALAYSIA: Johor: Kota Tinggi, 20 Aug 1954, Sinclair, J. 8151 (E); ibidem, 20 Aug 1954, Sinclair, J. SFN40358 (SING); Bekok, Endau State Park, Sungai Selai, 200 m, 16 Aug 2002, Sam, Y.-Y. FRI47104 (KEP); Bekok, Endau State Park, Sungai Selai, 200 m, 16 Aug 2002, Sam, Y.-Y. FRI47104 (KEP); Kedah: Weng, 3 Aug 1985, Bogner 1699 (M); Gunung Inas Recreation Park, 29 May 2001, Sam, Y.-Y. FRI46549 (KEP); Baling, Gunung Inas Forest Reserve, Bukit Iboi, 2 Nov 2007, Imin, K., Kueh, H.L. \& Phoon, S.N. et al. FRI58594 (KEP, SING); Kelantan: Gua Panjang, 7 Aug 1962, UNESCO limestone expedition 440A (K, L, SING); Gua Musang, Kuala Betis, 8 Oct 1985, Latiff, A. \& Zainudin, A. ALM1033 (L); Sg. Ketil, May 1991, Davison, G.W.H. s.n. (KEP); Jeli, Kuala Yong, 22 Sep 1986, Latiff, A., Zainudin, A. \& Miran, S. ALM1644 (UKMB); Kelumpor, 3 Feb 1923, Md Haniff \& Md Nur 10390 (SING); Kuala Krai, Stong FR, 8 Aug 2009, Julius, A. \& Imin, K. FRI56237 (KEP); Melaka: unknown loc., Cuming, H. 2387 (K); ibidem, Griffith, W. s.n. (K); ibidem, 20 Aug 1916, Burkill, I.H. 2159 (SING); Sungei Rambei River, Jun 1889, Derry, R. 205 (SING); ibidem, Oct 1889, Derry, R. 305 (SING); 
Selandar, Oct 1893, Goodenough, J.S. 1518 (SING); Negeri Sembilan: Gunung Angsi, 7 Sep 1937, Franck, C.W. 1150 (C); Selam Forest Reserve, 27 Nov 1922, Holttum, R.E. 9715 (SING); Bukit Sutu, 1 Nov 1885, Alvins, M.V. 1942 (SING); Jelebu, Berembun FR, Jeram Toi, 446 m, 8 Apr 2008, Yao, T.L., Angan, A. \& Norazmi, A. FRI57913 (KEP); Pahang: Tahan River, 150 m, 8 Sep 1937, Corner, E.J.H. s.n. (L); Sungei Takan, Aug 1891, Ridley, H.N. s.n. (SING); Puku, Kuala Teku, 21 Dec 1920, Seimund, E. 390 (SING); Sungai Sat, 18 Jul 1929, Henderson, M.R. SFN21928 (BM, NY, SING); Jerantut, Tekam Forest Reserve, 3 Oct 2002, Sam, Y.-Y. FRI44492 (KEP); Tasek Bera, 16 Mar 1939, Mashall 35847 (KEP); Jerantut, Tekam Forest Reserve, 3 Oct 2002, Sam, Y.-Y. FRI44492 (KEP); Penang: Government Hill, Jul 1894, Curtis, C. s.n. (SING); Penang Hill, 500 m, 14 Aug 1986, Weber, A. s.n. (KEP); Beside Hill railway, 14 Aug 1925, Flippance, F. s.n. (SING); Perak: Changkat Mentri, Sep 1918, Kloss, C.B. 6476 (K); Kuala Kangsar, Bubu Forest Reserve, Sungei Kenas, 410 m, 22 Oct 1992, Saw, L.G. FRI37684 (KEP); Sunkai, 24 Feb 1987, Anthony, S. SA740 (KEP); Perak River, 14 Jan 1963, Allen, B.M. 4852A (SING); Sungai Ryah, Scortechini, B. 37 (SING); Temengor, 1909, Ridley, H.N. 14281 (SING); ibidem, Jul 1909, Ridley, H.N. 14282 (BM); Taiping, Bukit Larut, Sep 1908, Long, F.R. 13 (K); ibidem, Keng, H. et al. 1 (SINU); Selangor: Ulu Langat, 19 Aug 1959, Gadoh anak Umbai for A.H. Millard KL1680 (K, KEP, L, SING); ibidem, 23 Aug 1958, Gadoh anak Umbai for A.H. Millard KL781 (KEP); Genting Sempah, 21 Oct 1921, Hume, H.L. 9228 (SING); Semenyih, 23 Jul 1921, Hume, H.L. 8253 (SING); Genting Highlands, Ulu Gombak, 10 Oct 1921, Hume, H.L. 8943 (SING); Sungei Buloh, Oct 1899, Goodenough, J.S. s.n. (SING); Terengganu: Bukit Besar, 760 m, 25 Aug 1901, Annandale, N. \& Robinson, H.C. s.n. (CGE, K); ibidem, 910 m, 3 May 1899, Gwynne-Vaughan, D.T. 410 (CGE); Hulu Terengganu, Sg. Cicir, 4 Aug 2007, Jutta, M. \& Kueh, H.L. FRI59582 (KEP); Hulu Terengganu, Tembat Forest Reserve, 5 Apr 2010, Mohd Hairul, M.A., Ong, P.T., O’Byrne, P. \& Mohd Nazri, A. et al. FRI70936 (KEP); ibidem, 30 Jul 2009, Rosdi, M., Kamarul Hisham, M. \& Angan, A. FRI66336 (KEP).

SINGAPORE: Bukit Timah, 12 Sep 1948, Sinclair, J. s.n. (P); ibidem, 26 Feb 1931, Md Nur SFN24637 (NY, SING); ibidem, 1891, Ridley, H.N. 2704 (K); ibidem, 12 Sep 1948, Sinclair, J. 5090 (E); ibidem, 1891, Ridley, H.N. 2704 (SING); ibidem, 1900, Ridley, H.N. s.n. (BM).

Notes. Aeschynanthus radicans is rather similar to rare forms of A. pulcher with hairs on the leaves but can be distinguished from it by the hairs on the ovary (glands only in A. pulcher).

11. Aeschynanthus rhododendron Ridl., J. Linn. Soc. 32: 500 (1896); Ridley, J. Straits Branch Roy. Asiat. Soc. 44: 15 (1905); Ridley, J. Asiat. Soc. Bengal 74 (2): 735 (1909); Ridley, Fl. Mal. Pen. 2: 499 (1923); Turner, Gard. Bull. Singapore 47: 244 (1997 ['1995’]); Middleton, Edinburgh J. Bot. 64: 418 (2007). - TYPE: Peninsular Malaysia, Perak, Taiping, Gunung Hijau, 1520 m, 1892, Ridley, H.N. s.n. (lectotype SING [SING0035553], designated by Middleton (2007)). (Fig. 15, 16)

Aeschynanthus longicalyx Ridl., J. Straits Branch Roy. Asiat. Soc. 44: 16 (1905); Ridley, J. Asiat. Soc. Bengal 74 (2): 735 (1909); Ridley, Fl. Mal. Pen. 2: 499 (1923); Henderson, Malay. Wild. Fl. Dicot. 341 (1959); Turner, Gard. Bull. Singapore 47: 243 (1997 ['1995’]). - TYPE: Malaysia, Selangor, Semangkok Pass, February 1904, BurnMurdoch s.n. (lectotype SING [SING0035404], designated by Middleton (2007); isolectotype $\mathrm{K})$. 
Aeschynanthus longicalyx var. superbus Ridl., J. Fed. Malay States Mus. 5: 43 (1914); Stone, Fed. Mus. J. 26 (1): 98 (1981); Middleton, Edinburgh J. Bot. 64: 422 (2007); Middleton, Edinburgh J. Bot. 66: 440 (2009). - TYPE: Peninsular Malaysia, Selangor, Gunung Mengkuang Lebah, 1520 m, 4 February 1913, Robinson, H.C. s.n. (lectotype SING [SING0035408], designated by Middleton (2007); isolectotypes BM [BM000537105], K [K000831889]).

Epiphyte with erect and arching stems; branchlets glabrous. Leaves opposite; petiole 3-12 mm long, glabrous; blade coriaceous to fleshy, mid to dark green above, paler beneath, not marbled, ovate or elliptic, $2.1-13 \times 0.9-5.9 \mathrm{~cm}, 1.3-5.8$ times as long as wide, apex acuminate, base cuneate to rounded, glabrous above and beneath, punctate or not beneath, margin entire, secondary veins obscure to clearly visible, 3-5 pairs, tertiary venation obscure or laxly reticulate. Inflorescences axillary or subterminal with 1-2 flowers; peduncle absent; bracts linear, c. $2 \mathrm{~mm}$ long; pedicels 8-19 $\mathrm{mm}$ long, glabrous. Calyx with a tube and free lobes, rarely slightly zygomorphic, red, sometimes with some green at base, glabrous or with a few hairs only on very tips of lobes, 16-65 mm long; tube $11-37 \mathrm{~mm}$ long which is $45-88 \%$ of total length, 7.5-27 mm wide at top of tube; lobes triangular or narrowly triangular, erect, 2.3-32 × 1.8-11 mm, apex acuminate or acute. Corolla 54-102 mm long, tube curved, narrow at base, externally bright red on tube and lobes, internally red with darker markings on lower 3 lobes and pale orange at base of lobes and in tube; upper lobes oblong, slightly spreading to reflexed, 9-22 × 8.5-12 mm, sinus 5-11 mm deep, apex rounded; lateral lobes ovate or orbicular, reflexed, 7-18 $\times 7-16 \mathrm{~mm}$, apex rounded; lower lobe oblong or obovate, spreading or reflexed, 9-21 $\times 8.5-12 \mathrm{~mm}$, apex rounded; glabrous to sparsely eglandular puberulent outside, sometimes only around top, inside with scattered glandular hairs throughout except at base and becoming more dense in throat, sessile glands inside tube present. Stamens not exserted or only very slightly exserted beyond upper lobes, fused in 2 pairs; filaments bright red, with glandular hairs; anterior filaments inserted in tube at $40-50.5 \mathrm{~mm}$ from corolla base which is $48-59 \%$ of corolla length, filaments $32-41 \mathrm{~mm}$ long, anthers $3.6-5 \times 1.4-2.5 \mathrm{~mm}$; posterior filaments inserted in tube at $51-56 \mathrm{~mm}$ from corolla base which is $62-69 \%$ of corolla length, filaments 20-25 mm long, anthers 3-4.5 × 1.1-2.3 mm; staminode 0.7-4 mm long. Disk 0.8-1.5 mm high, a simple annular ring or 5-crenate. Pistil 5684 mm long; stipe 18-33 mm long, glabrous; ovary 20-50 mm long, glabrous; style 7-22 mm long, densely glandular and eglandular pubescent. Capsule 11-22 cm long, 4-6 mm wide, with a long stipe. Seed grain 0.8-1.5 × 0.2-0.4 mm, papillose, bubble cells absent; apical appendage short and stout, 0.7-1.2 mm long; hilar appendage a single stout appendage, 0.7-1.1 mm long; appendages not papillose.

Distribution. Extreme south of Thailand, Peninsular Malaysia (Kelantan, Pahang, Perak, Selangor), Sumatra.

Habitat and ecology. Recorded from (90-)1200-2100 m altitude. Almost all collections of this species have been made in upper montane forest at over $1200 \mathrm{~m}$ altitude or in 


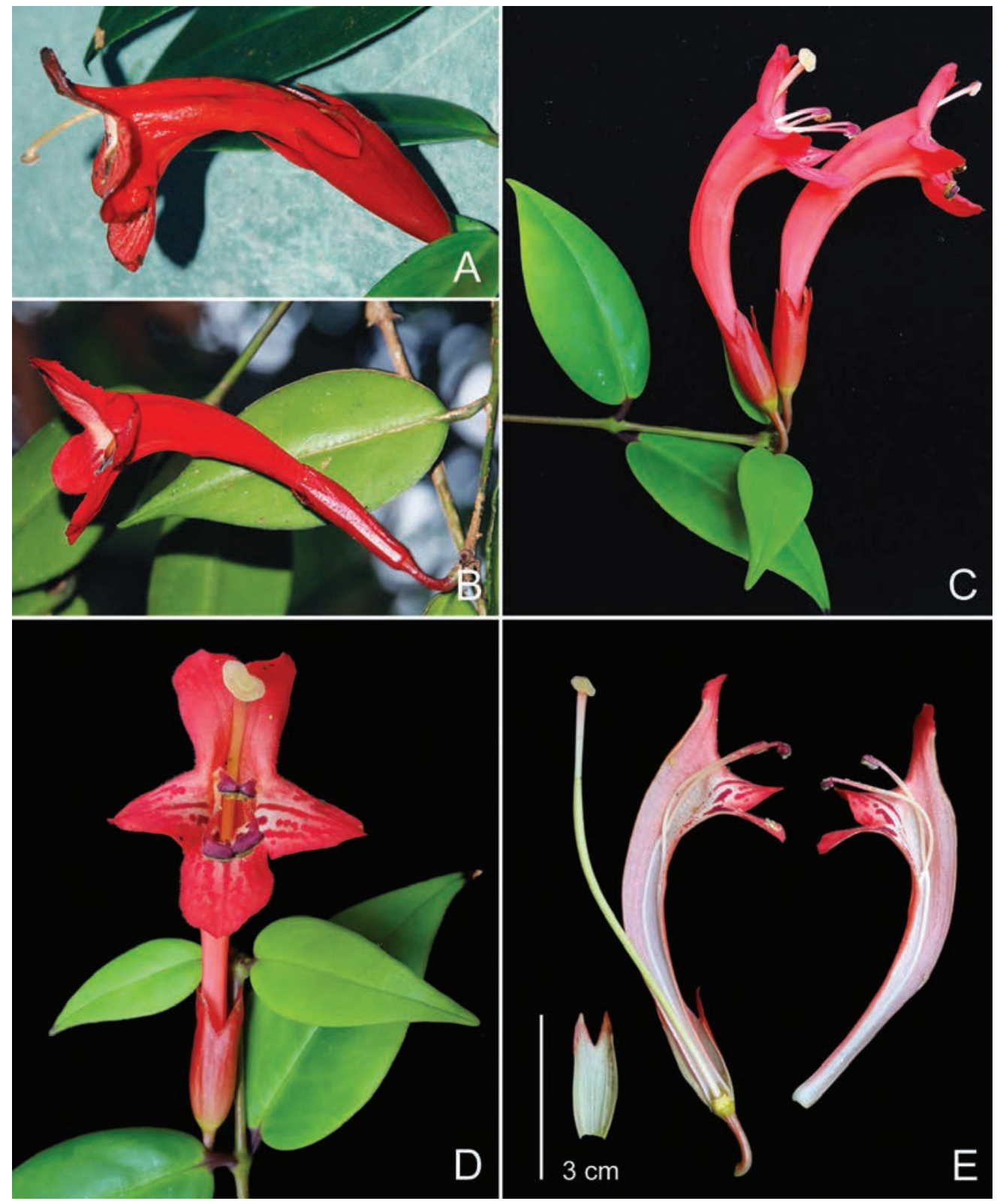

Fig. 15. Aeschynanthus rhododendron Ridl. A. Flower with larger-lobed calyx from southern part of range. B. Flower with smaller-lobed calyx from northern part of range. C. Habit showing the stamens beginning to wither and reflex in the flower on the right. D. Flower from the front. E. Flower dissection. (Photos: A-B, David Middleton; C-E, Jana Leong-Škorničková)

mossy forest at even higher altitudes. However, there is a collection, Mohd Shah \& Sidek MS1073 (E, SING) recorded from much lower altitude, 300-400 feet (90-120 m) at Bukit Larut. This may be a mistake. 


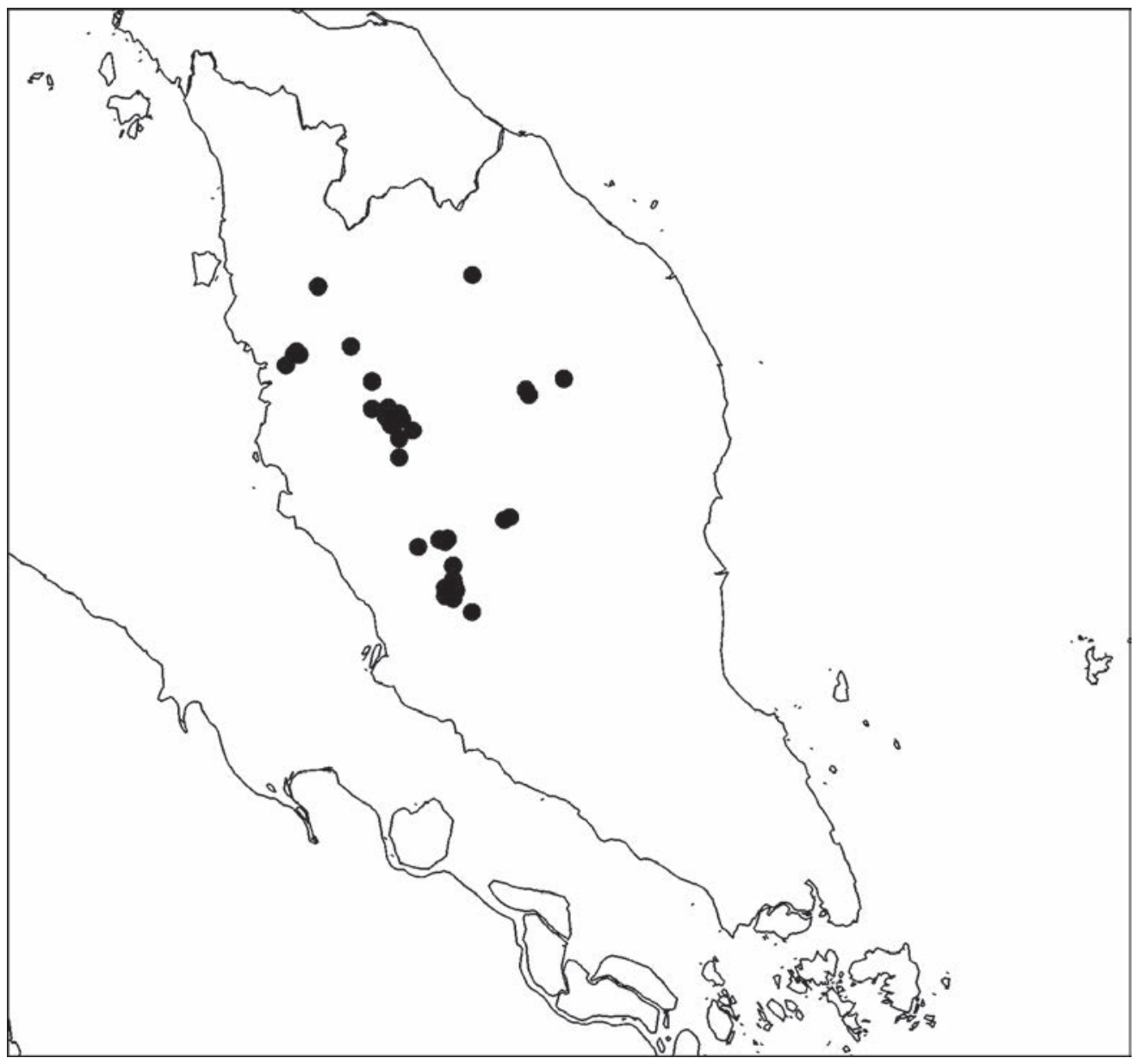

Fig. 16. Distribution of Aeschynanthus rhododendron Ridl. in Peninsular Malaysia (•).

Provisional IUCN conservation assessment. Least Concern (LC). This species is widespread and locally common.

Additional Peninsular Malaysian specimens examined. PENINSULAR MALAYSIA: Kelantan: Kuala Krai, G. Tera, Permatang C, 11 Feb 2007, Yao, T.L., Kiew, R., Chew, M.Y. \& Kamarudin S. FRI55845 (KEP); Pahang: Genting Highlands, 25 Nov 1984, Kiew, R. RK1546 (KEP); ibidem, 15 Feb 1987, Worthington, R.D. 12463 (L, NY); ibidem, 1520 m, 14 Jun 1967, Whitmore, T.C. FRI3887 (KEP, L, SING); Genting Highlands, Trail to Bt. Tunggul, 1500 m, 18 Jan 1994, Perumal, B., Gan, C.L., Angan, A. \& Bedul FRI41661 (KEP); Genting Highlands, Gunung Ulu Kali, 25 Mar 2008, Phoon, S.N. \& O’Byrne, P. et al. FRI60557 (K, KEP); ibidem, 9 May 1991, Tan, W.K. et al. TWK1 (SING); ibidem, 1670 m, 19 Sep 1967, Dransfield, J. s.n. (KLU); ibidem, 1500 m, 21 Dec 1982, Stone, B.C.M. 15366 (KLU); ibidem, 1767 m, 25 Mar 2008, Phoon, S.N. FRI60496 (K, KEP); ibidem, 1700 m, 24 Sep 1998, Kamarudin S. FRI33755 (KEP); ibidem, 1750 m, 12 Oct 1974, Van Balgooy, M.M.J. 2154 (E, L); ibidem, 1670 m, 18 Mar 1979, Stone, B.C.M. 14045 (KLU, L); ibidem, 1700 m, 4 Jun 1977, Siew Wei Hoe 47 (L); 
ibidem, 2 Mar 1996, Van Balgooy, M.M.J. 7133 (K, KEP, L); ibidem, 14 Mar 1982, Stone, B.C.M. 15071 (BISH); ibidem, 1756 m, 22 Mar 2006, Syahida-Emiza FRI51460 (KEP, SING); ibidem, 16 Feb 2007, Wilkie, P., Julius, A., Nor Ezzawanis, A.T. \& Imin, K. FRI52906 (KEP); Cameron Highlands, 1976, Anthony, S. SA231 (KEP); ibidem, 30 Mar 1932, Mead, J.P. 27945 (SING); ibidem, 9 Mar 1947, Wyatt-Smith, J. KEP 56945 (K, KEP, L); ibidem, 1820-2000 m, Aug 1975, Keng, H. et al. K8010 (SINU); ibidem, Batten Pooll, A.H. s.n. (SING); ibidem, 2120 m, 1 Feb 1962, Togashi, M. 622111 (TI); ibidem, 2000 m, 5 Mar 1970, Togashi, M. s.n. (TI); ibidem, 1820-2000 m, 25-31 Aug 1975, Rao \& et al. K8010 (AAU, L); Cameron Highlands, Ulu Bertam Forest Reserve, 1450 m, 9 Nov 1959, Abbe, E.C., Kadim bin Tassim, Mansor bin Omar \& Abbe, L.B. 9119 (L, NY); Cameron Highlands, Gunung Batu Berinchang, Tarred road to microwave station descending $11 \mathrm{~km}$ to Brinchang, $2000 \mathrm{~m}, 7$ May 2007, Yao, T.L., Nooteboom, H.P. \& Angan, A. FRI55898 (KEP); Cameron Highlands, Gunung Batu Berinchang, 1620 m, 20 Jan 2010, Mohd Hairul, M.A., Ong, P.T., Siti Munirah, M.Y. \& Kueh, H.L. FRI69938 (KEP); ibidem, 1950 m, 20 Jan 2011, Imin, K., Mohd Nazri, A. \& Julius, A. et al. FRI71954 (KEP); ibidem, 9 Apr 1987, Saw, L.G. FRI34368 (KEP); ibidem, 22 Aug 1995, Tam Sheh May TSM2 (KEP); ibidem, 1997 m, 11 Feb 2010, Saw, L.G., Damahuri, S. \& Norzamli, A. FRI48241 (BKF, KEP); ibidem, 2020 m, 30 Aug 1970, Chin, S.C. 233 (KLU); ibidem, 1980 m, 22 Mar 2007, Julius, A. FRI56020 (KEP, SING); ibidem, 5 Nov 1960, Poore, M.E.D. 466 (KLU); ibidem, 30 Jul 1967, Stone, B.C.M. 7218 (KLU); ibidem, 29 Jul 2007, Low, Y.W. LYW140 (KLU); ibidem, 1920 m, 9 Dec 1961, Abdul Samat bin Abdullah 68 (KLU); ibidem, 2024 m, 26 Oct 2007, Imin, K. et al. FRI58570 (K, KEP, SING); ibidem, 1945 m, 21 Feb 2008, SyahidaEmiza FRI57293 (K, KEP, SING); ibidem, 1910 m, 10 Jun 1961, Castle-Smith, P.M. 42 (K); ibidem, 17 Mar 1996, Van Balgooy, M.M.J. 7228 (KEP, L); ibidem, 1910 m, 18 Apr 1968, Woods, P.J.B. 680 (E); ibidem, 1870 m, 26 Jul 2002, Bramley, G. \& Neale, S. GB31 (E, K, KEP); ibidem, 2000 m, 20 Jun 1975, Van Balgooy, M.M.J. 2663 (E, L); ibidem, 13 Sep 1985, Latiff, A., Zainudin, A. \& Miran, S. ALM970 (L); ibidem, 1880 m, 12 Aug 1986, Wong, K.M. FRI35244 (K, KEP, L, SING); ibidem, 2020 m, 4 Nov 1958, Sinclair, J. 9949 (E, SING); ibidem, 1970 m, 11 Oct 1963, Chew, W.-L. CWL922 (K, L, SING); ibidem, 2020 m, 7 Mar 1960, Hawkins, A.S.M. 6 (SING); ibidem, 2 Apr 1989, Latiff, A. \& Zainudin, A. ALM3121 (UKMB); ibidem, 1520 m, 15 Feb 1975, Mohd Shah MS 3446 (SING); ibidem, 8 Sep 1970, Whitmore, T.C. FRI15450 (K, KEP, L, SING); ibidem, 1820 m, 9 Apr 1930, Henderson, M.R. SFN23534 (NY, SING); ibidem, 2020 m, 8 Apr 1960, Abbe, L.B., Abbe, E.C., Kadim bin Tassim \& Mansor bin Omar 9767 (K, L, NY); Gunung Benom, 14 Nov 2009, Mohd Hairul, M.A. \& Mohd Nazri, A. et al. FRI69892 (KEP); Cameron Highlands, Gunung Berembun, 1750 m, 5 Apr 1988, Sabari, D. FRI32715 (KEP); ibidem, 28 Jun 1988, Samsuri Ahmad SA96 (SINU); ibidem, 9 Aug 2008, Rosdi, M. \& Phoon, S.N. et al. FRI59867 (KEP); ibidem, Nov 1908, Ridley, H.N. 13600 (BM, K, SING); ibidem, 1820 m, 25 Nov 1925, Henderson, M.R. 17988 (SING); ibidem, 4 Apr 1930, Henderson, M.R. s.n. (SING); ibidem, 1820 m, 10 Sep 1970, Whitmore, T.C. FRI15495 (K, KEP, L, SING); ibidem, 1670 m, 3 Oct 1963, Chew, W.-L. CWL772 (C, G, K, L, SING); Cameron Highlands, Trail to G. Berembun, 1670 m, 1 Mar 1968, Ng, F.S.P. FRI5931 (K, KEP, L); Cameron Highlands, near Parit Falls, Mar 1952, Johnston, A.M. 76 (SING); Cameron Highlands, Break Pressure Tank Hill, 1480 m, 31 Aug 1956, Burkill, H.M. HMB760 (K, L, LAE, SING); Cameron Highlands, Tanah Rata, 1400-1500 m, 12 Jan 1983, Davis 69248A (E [2 sheets], SING); ibidem, 1440 m, 29 Aug 1956, Burkill, H.M. HMB730 (K, L, SING); Cameron Highlands, Tanah Rata, Parit Waterfall, 28 Aug 1990, Okada, H., Darnaedi, D., Akiyama, H., Kawahara, T. \& Watano, Y. 1027 (TI); Cameron Highlands, Robinson's Falls, 1430 m, 24 Mar 2007, Julius, A., Middleton, D.J. \& Lindsay, S. et al. FRI57483 (KEP); ibidem, 1400-1840 m, 20 Mar 1992, Klackenberg, J. \& Lundin, R. 689 (L); 
ibidem, 9 May 1964, Mahmud bin Sidek 4816 (KLU); ibidem, 14 Apr 1968, Woods, P.J.B., Black, M. \& Wycherley, P. 615 (E, KEP); Cameron Highlands, Tanah Rata, 1440 m, 29 Aug 1970, Chin, S.C. 165 (KLU); Cameron Highlands, Brinchang Mist Forest, 1670 m, 20 Feb 1962, Poore, M.E.D. 1028 (KLU); Gunung Benom, 31 Jul 1925, unknown s.n. (SING); ibidem, 28 Jul 1925, Federated Malay States Museum Coll s.n. (K); Gunung Mengkuang, 1520 m, 28 Mar 1959, Wyatt-Smith, J. 78808 (KEP); Gunung Tahan, Tangga Duabelas, 1530 m, 24 Mar 1987, Kiew, R. RK2430 (KEP); Above Genting Sempah, 1440 m, 23 Jul 1967, Stone, B.C.M. 7194 (KLU, L); Fraser's Hill, 15 Nov 1977, Keng, H. et al. 410 (SINU); ibidem, 1450 m, 27 Aug 1923, Henderson, M.R. 11365 (SING); Fraser's Hill, Pine tree hill, 1450 m, 19 Sep 1922, Burkill, H.M. \& Holttum, R.E. 8531 (SING); ibidem, 1450 m, 27 Aug 1923, Md Nur 11056 (SING); Gunong [Gunung] Gedong, 3 Sep 1928, Holttum, R.E. 20769 (SING); Gunung Tahan, 1911, Ridley, H.N. 16090 (K, SING); Perak: unknown loc., Scortechini, B. s.n. (SING); Caulfield's Hill, 1210 m, Apr 1884, Scortechini, B. 388 (SING); ibidem, Aug 1885, Wray, L. 656 (K, SING); Taiping, Scortechini, B. $468 b$ (SING); ibidem, 12 Nov 1889, Curtis, C. s.n. (SING); Taiping, Gunung Hijau, 1530 m, 7 Jun 1983, Stone, B.C.M. 15497 (KLU); ibidem, 7 Mar 1939, Spare, G.H. 2047 (SING); ibidem, Sep 1889, Curtis, C. s.n. (SING); ibidem, 1448 m, 19 Mar 2007, Julius, A., Middleton, D.J. \& Lindsay, S. et al. FRI53302 (KEP, SING); ibidem, 1448 m, 14 Jul 2006, Kamarul Hisham, M. FRI52054 (KEP); ibidem, 1440 m, 5 Mar 1924, Henderson, M.R. 11829 (SING); ibidem, 1360 m, 14 Feb 1907, Md Haniff \& Md Nur 2456 (SING); ibidem, 8 Oct 1899, Fox, W. s.n. (SING); ibidem, 1210 m, Dec 1887, Curtis, C. 1311 (K, SING); ibidem, 1210-1360 m, 4 Dec 1965, Mohd Shah \& Sidek MS1110 (SING); ibidem, 1520 m, 1892, Ridley, H.N. s.n. (SING); ibidem, 1380 m, 11 Sep 1949, Sinclair, J. \& Kiah SFN38670 (BM, E, K, L, P, SING); Sungei Pelus, 1520 m, 1889, Wray, L. s.n. (SING); Gunong [Gunung] Inas, 1180-1360 m, 9 Dec 1899, Yapp, R.H. 440 (CGE, K); Gunung Batu Puteh, 1920 m, 26 Feb 1994, Perumal, B., Gan, C.L., Shahril, K.Z. \& Angan, A. FRI41602 (KEP, KLU); Gunong [Gunung] Jasar, 23 Aug 1977, Ng, F.S.P. FRI27146 (KEP); Gunong [Gunung] Jasar, 17 Apr 1968, Woods, P.J.B. 632 (E); Gunung Korbu, 1670 m, 10 Mar 1913, Robinson, H.C. s.n. (K); Taiping, Bukit Larut, Bukit Bintang Hijau, 1451 m, 1 Mar 2011, Wilkie, P., Siti Munirah, M.Y., Mohd. Hairul, M.A. \& Nazre FRI75005 (E, KEP); ibidem, 16 Aug 1986, Weber, A. s.n. (KEP); ibidem, 90-120 m, 9 Dec 1965, Mohd Shah \& Sidek MS 1073 (E, SING); ibidem, 1270 m, 20 Oct 1988, Saw, L.G. FRI36398 (KEP); ibidem, 27 Jun 1984, Keng, H. et al. D-\#5 (SINU); Selangor: Semangkok Pass, Feb 1904, Burn-Murdoch s.n. (K, SING); Gunung Mengkuang Lebah, 1520 m, 16 Jan 1913, Robinson, H.C. s.n. (K); ibidem, 1520 m, 17 Jan 1913, Robinson, H.C. s.n. (K); ibidem, 1520 m, 4 Feb 1913, Robinson, H.C. s.n. (BM, K, SING); Bukit Fraser, Jul 1899, Hose, G. 46 (SING); Gunong [Gunung] Bunga Buah, 1360m acc. no. 19680624 vouchered as Cultivated C7315 (E, KEP); Ulu Langat, Feb 1912, Kloss, C.B. s.n. (K); Hulu Langat, Ulu Langat Forest Reserve, 1440 m, 25 Jan 2003, Sam, Y.-Y. FRI47208 (KEP); Ulu Langat, Gunung Nuang, 12 May 1940, Symington, C.F. 51762 (KEP); Gunong [Gunung] Nuang, 15 Aug 1974, Lee, D.W. s.n. (KLU); Ulu Selangor, Gunung Moyang, 3 Nov 1940, Symington, C.F. 56711 (KEP).

Notes. This species is rather variable in the shape of the calyx. The type of the species is from Gunung Hijau in the Taiping Hills where the calyx is generally narrow and the lobes small. This is the form also found in Thailand. In the Genting Highlands, Fraser's Hill and Gunung Benom the calyx tube flares gently from the base so that the mouth is wider and the lobes are much larger. This is the plant formerly recognised as Aeschynanthus longicalyx. The largest calyx seen is from Gunung Ulu Kali in the Genting Highlands. Material from the Cameron Highlands is rather intermediate. 
12. Aeschynanthus speciosus Hook., Bot. Mag. 73: t.4320 (1847); Miquel, Fl. Ned. Ind. 2: 718 (1858); Clarke in A.DC. \& C.DC., Monogr. Phan. 5(1): 33 (1883); Ridley, J. Linn. Soc. Bot. 32: 499 (1896); Ridley, J. Straits Branch Roy. Asiat. Soc. 44: 14 (1905); Ridley, J. Asiat. Soc. Bengal, Pt. 2, Nat. Hist. 74(2): 733 (1909); Ridley, Fl. Mal. Pen. 2: 498 (1923); Turner, Gard. Bull. Singapore 47(1): 244 (1997 ['1995’]); Burtt, Thai Forest Bull., Bot. 29: 84 (2001); Smitinand, Thai Pl. Names, ed. 2: 15 (2001); Middleton, Edinburgh J. Bot. 64: 420 (2007). - Trichosporum speciosum (Hook.) Kuntze Revis. Gen. Pl.: 478 (1891). - TYPE: Java, Jawa Barat, Mt Asapan, Lobb, T. s.n. (lectotype K, designated by Middleton (2007)). (Fig. 17, 18)

Aeschynanthus aucklandiae Low, Sarawak: 386 (1848). - TYPE: Borneo, East Malaysia, Sarawak, Low, H. s.n. (holotype CGE).

Epiphyte with erect, arching and pendulous stems; branches glabrous. Leaves in whorls of 3-6; petiole generally wide and flat, sometimes slightly winged, 2-12 mm long, glabrous; blade coriaceous or slightly fleshy, mid to dark green above, paler beneath, not marbled, elliptic or ovate, 3.8-15.3 × 1.2-5.5 cm, 2.3-6.1 times as long as wide, apex acuminate to caudate, base cuneate to rounded, glabrous above and beneath, not punctate beneath, margin dentate to entire, often strongly undulate, secondary veins obscure to weakly visible, c. 7-8 pairs, tertiary venation obscure. Inflorescences terminal with 4-12 flowers; peduncle absent; bracts linear, 5-13 mm long; pedicels 7-14.5 mm long, glabrous or sparsely eglandular puberulent. Calyx of separate lobes free to the base, pale green, yellowish or dark reddish brown and then often flushed with one of the other colours, glabrous to densely eglandular or glandular puberulent; lobes narrowly triangular or linear, erect, 6.5-26.3 × 1-2.6 mm, apex acute or acuminate. Corolla 54-118 mm long, tube narrow at base, externally yellow, yellowish green or orangish on basal half to two-thirds of tube and orange-red to bright red above, more rarely bright red all over, lobes orange-red to bright red, internally light yellowish in tube and red or orangish on upper 2 lobes and red or orangish at the margin with darker $\mathrm{V}$ - or $\mathrm{W}$-shaped markings on lower 3 lobes and yellowish at the base; upper lobes oblong, not spreading or reflexed, 4.5-11 × 3.6-8 mm, sinus 4-8.2 mm deep, apex rounded; lateral lobes oblong, deltoid or ovate, spreading, 5.7-14 × 5.7-13.5 $\mathrm{mm}$, apex rounded; lower lobe oblong or elliptic, spreading or reflexed, $7-15.5 \times$ 4.5-12 mm, apex retuse to rounded; outside glabrous, slightly papillose or sparsely glandular puberulent, sometimes only on ciliate lobes, inside with sparse sessile glands. Stamens long exserted, fused in 2 pairs; filaments bright red or white, with glandular hairs, anthers grey, pale brown or purple-black; anterior filaments inserted at $50-96 \mathrm{~mm}$ from corolla base which is $67-81 \%$ of corolla length, filaments $29-46 \mathrm{~mm}$ long, anthers 3.9-7 × 1.3-2.3 mm; posterior filaments inserted at 53-100 mm from corolla base which is $72-85 \%$ of corolla length, filaments $21-38 \mathrm{~mm}$ long, anthers 2.6-5 × 1.2-2.1 mm; staminode 0.3-4 mm long. Disk 1.2-2.5 mm high, 5-dentate or 5-crenate. Pistil 83-130 mm long; stipe 20-30 mm long, with few sessile glands or glabrous; ovary 27-44 mm long, with sessile glands, these sometimes very few; style yellow or green, 14-65 mm long, glandular pubescent, especially in upper half. 
Capsule 20-45 cm long, 2.3-3.6 mm wide. Seed grain 0.9-1.3 × 0.3-0.4 mm, warty, bubble cells absent; apical appendage a filiform hair, 15-22 mm long; hilar appendage a single filiform hair, 15.5-23 mm long; appendages papillose.

Distribution. Southern Thailand, Peninsular Malaysia (Pahang, Perak, Selangor), Sumatra, Java, Borneo.

Habitat and ecology. In hill dipterocarp forest habitats at 240-760 m altitude. It has been recorded to $1900 \mathrm{~m}$ altitude in Sumatra and is likely to also occur at higher altitudes in Peninsular Malaysia.

Provisional IUCN conservation assessment. Least Concern (LC). This species is remarkably infrequently collected despite having very showy flowers such that it is readily observed in the forest for collection. It is likely to be relatively rare over most of its range but both the $\mathrm{EOO}$ and $\mathrm{AOO}$ are much higher than those suggesting a threat category would be merited.

Peninsular Malaysian specimens examined. PENINSULAR MALAYSIA: Pahang: Bentong Sg., Naming, 760 m, 18 Jan 1959, unknown KEP93108 (KEP); Rompin, Endau-Rompin State Park, Ulu Kinchin, Sg. Kinchin, Norain, Raihana \& Rosjana 07 (SINU); Perak: Near Ulu Selama, 8 Jan 1900, Yapp, R.H. 610 (CGE); Bruseh near Bidor, 240 m, 27 Jun 1904, Napier, W. s.n. (SING); Selangor: Ulu Langat, Gadoh, U. KL1319 (KEP, SING); Serendah Ridge, 300 m, 18 Jul 1957, Hawkins, A.S.M. s.n. (SING); Bukit Elam, Jan 1891, Kelsall, H. s.n. (SING).

Notes. It is possible that Aeschynanthus pseudohybridus Mendum from Borneo is a synonym of this species but further study is necessary. It differs in the more uniformly red flowers compared to the orange and yellow flowers of $A$. speciosus.

13. Aeschynanthus volubilis Jack, Trans. Linn. Soc. London 14: 42 (1823); Brown, Cyrtandreae 115 (1839); Steudel, Nomencl. Bot. ed. 2, 1: 32 (1840); Brown in Bennett, Pl. Jav. Rar. 115 (1840); A.DC., Prod. 9: 262 (1845); Miquel, Fl. Ned. Ind. 2: 719 (1858); C.B.Clarke in A.DC. \& C.DC., Monogr. Phan. 5(1): 46 (1883); Bakhuizen van den Brink, Blumea 6: 395 (1950); Merrill, J. Arnold Arb. 33: 214 (1952); Backer \& Bakhuizen van den Brink, Fl. Java 2: 524 (1965); Sinha, Fl. Gt. Nicobar Isl. 329 (1999). - Trichosporum volubile (Jack) Nees, Flora 8: 144 (1825). - TYPE: Plate II, fig. 3 in Jack, Transactions of the Linnean Society of London vol. 14, unnumbered page between pp. 44 and 45 (1823), lectotype designated here. Epitype: Sumatra, East Coast [Sumatera Utara], Asahan, Silo Maradja, June 1927, Bartlett 8695 (epitype NY, designated here; isoepitype MICH). (Fig. 19)

Aeschynanthus obovatus C.B.Clarke in A.DC. \& C.DC., Monogr. Phan. 5(1): 47 (1883). - Trichosporum obovatum (C.B.Clarke) Kuntze, Revis. Gen. Pl. 478 (1891). TYPE: Kalimantan, Kalimantan Selatan, Banjarmasin, Motley, J. 1158 (holotype K). 

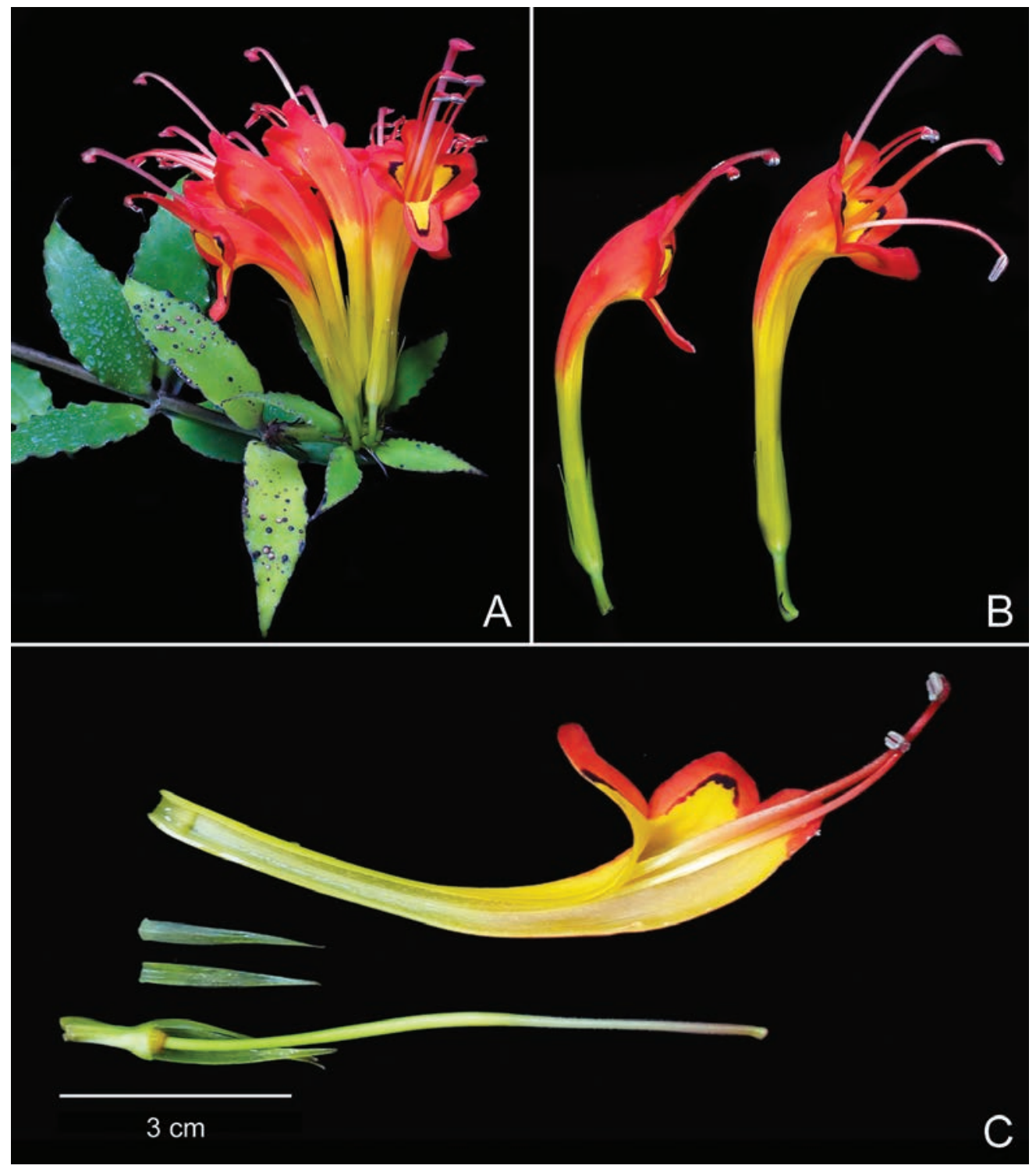

Fig. 17. Aeschynanthus speciosus Hook. A. Habit. B. Flowers, side view. The flower on the right is older with a much longer style and the stamens beginning to reflex (and with one pair becoming detached). C. Flower dissection with 2 calyx lobes removed to view nectary. (Photos: Jana Leong-Škorničková)

Aeschynanthus obovatus var. pallidus C.B.Clarke in A.DC. \& C.DC., Monogr. Phan. 5(1): 48 (1883). - TYPE: Java, Zippelius, A. Leiden number 52 (lectotype L [L0281661], designated here; isolectotypes L [L0281673, L0281674]).

Aeschynanthus hoseanus Kraenzl., J. Linn. Soc. Bot. 37: 284 (1906). - Trichosporum hoseanum (Kraenzl.) Merr., J. Straits Branch Roy. Asiat. Soc. special number: 530 


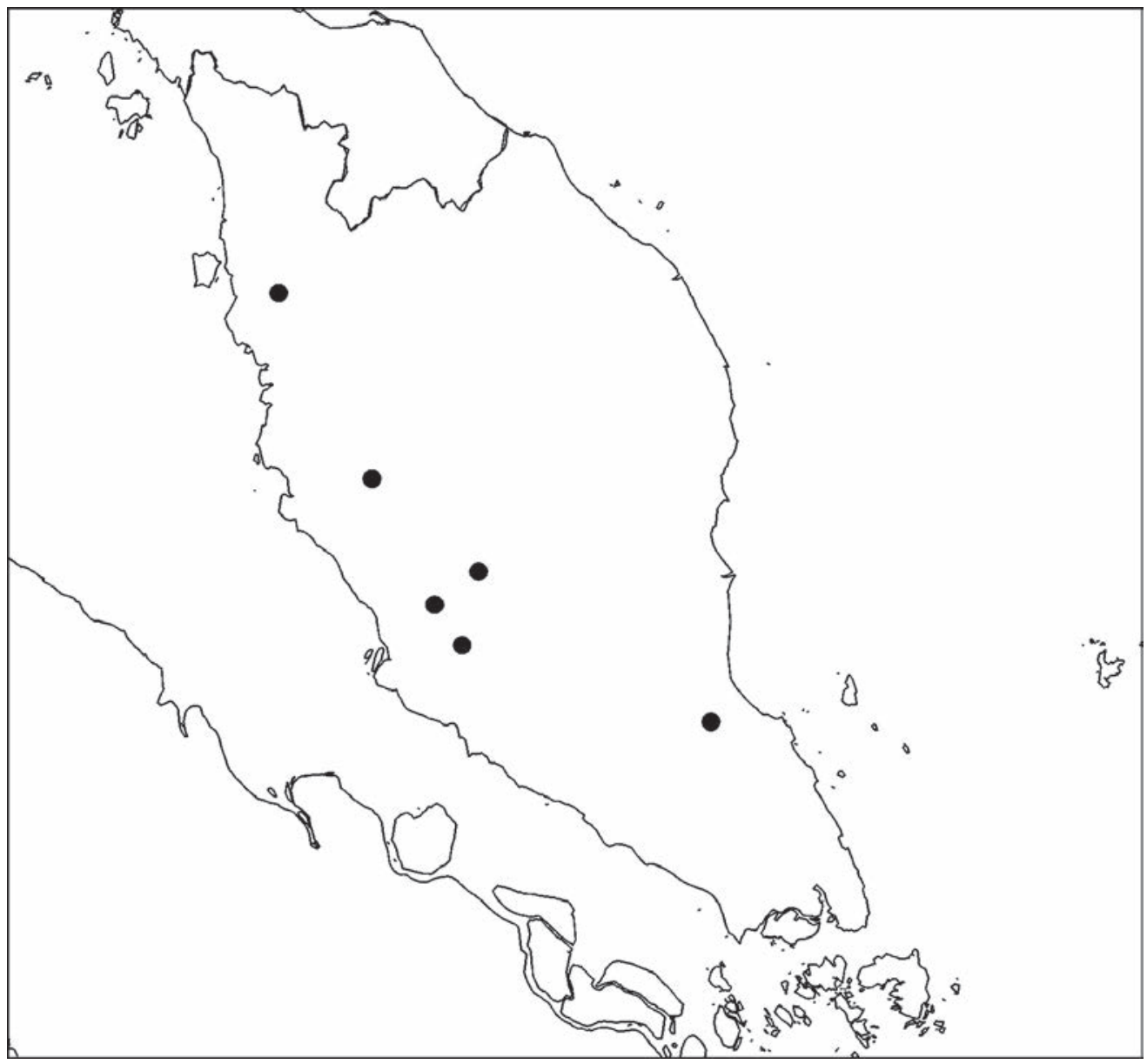

Fig. 18. Distribution of Aeschynanthus speciosus Hook. in Peninsular Malaysia (•).

(1921). - TYPE: Borneo, East Malaysia, Sarawak, Saribas, 24 Nov 1893, Haviland, G.D. \& Hose, C. 3528 (holotype K; isotype BM [BM000797673]).

Aeschynanthus flippancei Ridl., Bull. Misc. Info. Kew. 1926: 473 (1926); Turner, Gard. Bull. Singapore 47: 243 (1997 ['1995']). - TYPE: Cultivated in Penang Botanic Gardens but originally from Malaysia, Penang, Balik Pulau, Flippance, F. s.n. (lectotype K, designated here; isolectotype SING [SING0035633]).

Epiphyte with erect, arching or pendulous stems; stem glabrous. Leaves opposite; petiole 3-14 mm long, glabrous or sparsely puberulent; blade coriaceous, mid to dark green above, paler beneath, elliptic or ovate, $1.8-5.7 \times 0.7-3.2 \mathrm{~cm}, 1.6-2.7$ times as long as wide, apex obtuse to acuminate, base rounded to cuneate, glabrous above and beneath, not punctate beneath, not variegated, margin entire, 3-5 pairs of secondary veins, weakly visible or obscure, tertiary venation obscure. Inflorescence subterminal 
or axillary, 3-4-flowered; peduncle 3-5 mm long; pedicels 7-12 mm long, glabrous. Calyx with a tube for most of length and free lobes, tube fairly broad and gently flaring from base, 10-16.5 mm long; tube 8.8-14 mm long, 83-90\% of total length, c. $6 \mathrm{~mm}$ wide at top of tube; lobes triangular or semicircular, slightly spreading or erect, 1-2.5 $\times 2.3-5 \mathrm{~mm}$, apex rounded to acute, rarely acuminate. Corolla $19.5-27 \mathrm{~mm}$ long, tube slightly curved or almost straight, broad at base; upper lobes oblong, not spreading or reflexed, 3.2-4.5 $\times 2.5 \mathrm{~mm}$, sinus $1.5-2 \mathrm{~mm}$ deep, apices rounded; lateral lobes deltoid, reflexed, 3-5.2 × 3.8-4.4 mm, apices rounded; lower lobe oblong, spreading, 4.2-5.5 $\times 3.2-4 \mathrm{~mm}$, apex rounded; outside glabrous except for ciliate lobes, inside with sparse glandular hairs in upper part of tube and more densely so on inside of lobes except near margin. Stamens long exserted, fused in 2 pairs; filaments with sparse short stalked glands throughout; anterior filaments inserted at 14-17 mm from corolla base which is $52-63 \%$ of corolla length, filaments $16-18 \mathrm{~mm}$ long, anthers $2.4 \times 1 \mathrm{~mm}$; posterior filaments inserted at $16-19 \mathrm{~mm}$ from corolla base which is $48-67 \%$ of corolla length, filaments $12.5-13 \mathrm{~mm}$ long, anthers $2-2.2 \times 1-1.6 \mathrm{~mm}$; staminode $0.4-1.3 \mathrm{~mm}$ long. Disk 1-1.2 mm high, a simple annular ring or 5-crenate. Pistil 18-32 mm long; stipe 1.7-8 mm long, glabrous; ovary 10-16 mm long, with very few sessile glands and appearing glabrous; style 4-8 mm long, glandular pubescent. Capsule 10-28 cm long, 2.8-3 mm wide. Seed grain $0.5-0.6 \times 0.1-0.2 \mathrm{~mm}$ wide, smooth, bubble cells present at base of hilar end; apical appendage a filiform hair, 4-8.5 $\mathrm{mm}$ long; hilar appendage a single filiform hair, 7.5-9 $\mathrm{mm}$ long.

Distribution. Peninsular Malaysia (Penang), Sumatra (but see note below).

Habitat and ecology. None of the collections I have seen of this species from Malaysia or elsewhere include habitat data or altitudinal range.

Provisional IUCN conservation assessment. Data Deficient (DD). The limits of the species and its distribution are unclear.

Additional Peninsular Malaysian specimens examined. PENINSULAR MALAYSIA: Penang: Balik Pulau, Flippance, F. s.n. (K, SING); Government Hill, May 1921, Md Haniff s.n. (SING).

Notes. Aeschynanthus volubilis is the type species of the genus. It is known in Peninsular Malaysia only from a small number of specimens formerly identified as Aeschynanythus flippancei.

There are a number of species and specimens from Borneo and eastern Malesia which appear similar to Aeschynanthus volubilis but require further study to ascertain whether they are synonyms and/or extensions of the distribution of the species.

14. Aeschynanthus wallichii R.Br., Cyrtandreae 116 (1839); Brown in Bennett, Pl. Jav. Rar. 116 (1840); de Candolle, Prod. 9: 263 (1845); Miquel, Fl. Ned. Ind. 2: 722 (1858); C.B.Clarke in A.DC. \& C.DC., Monogr. Phan. 5(1): 48 (1883); Clarke in 
Hooker, Fl. Brit. Ind. 4: 343 (1884); Ridley, J. Linn. Soc. Bot. 32: 502 (1896); Ridley, J. Asiat. Soc. Bengal 74 (2): 736 (1909); Ridley, Fl. Mal. Pen. 2: 500 (1923); Turner, Gard. Bull. Singapore 45: 92 (1993); Turner, Gard. Bull. Singapore 47: 244 (1997 ['1995’]). - Aeschynanthus radicans Wall., Num. List no. 798 (1829), nom. nud. TYPE: Singapore, 1822, Wallich, N. 798 (lectotype K-W [K001111917], designated here; isolectotypes CGE, K [K000831885]). (Fig. 12, 19)

Aeschynanthus brevicalyx Miq., Fl. Ned. Ind. 2: 720 (1858); C.B.Clarke in A.DC. \& C.DC., Monogr. Phan. 5(1): 49 (1883). - Trichosporum brevicalyx (Miq.) Kuntze, Revis. Gen. Pl. 477 (1891). - TYPE: Indonesia, Sumatra, Sikilieh, Teijsmann, J.E. HB1194 (lectotype U, designated here; isolectotype L).

Epiphytic; stems glabrous. Leaves opposite; petiole 5-12 mm long, glabrous; blade coriaceous or slightly fleshy, ovate or elliptic, $3.1-10.5 \times 0.8-5 \mathrm{~cm}, 1.7-3.8$ times as long as wide, apex acuminate, base cuneate to subcordate, not marbled, glabrous above and beneath, not variegated, margin glabrous, entire, 4-5 pairs of secondary veins, only weakly visible or obscure, tertiary venation obscure. Inflorescence subterminal or axillary, 3-6-flowered; peduncles to $2.5 \mathrm{~mm}$ long, subglabrous to densely eglandular puberulent; pedicels 4.2-9 mm long, green, subglabrous to densely eglandular puberulent. Calyx fused into a wide open cup with lobes semicircular or only as curves on rim, green or yellow-green, sparsely to densely eglandular puberulent, sparsely pubescent inside, 2.5-6.5 mm long; tube 1.9-5.5 mm long which is 70-90\% of total length, tube 4-7 mm wide at apex; lobes semicircular or a weak curve of the rim, spreading or erect, 0.6-1.2 × 3.5-5.5 mm, apices rounded. Corolla 19.5-27 mm long, tube slightly curved or more or less straight, externally bright or dark red, base of tube not gibbose, outside sparsely to densely glandular to eglandular puberulent, inside with short stiff upward pointing hairs near base or throughout, sessile glands at inside sinuses of lobes; upper lobes orbicular or oblong, not spreading or reflexed, 3.8-6.6 $\times$ 3-4.5 mm, sinus 2.2-3.1 mm deep, apices rounded; lateral lobes ovate or deltoid, not spreading or reflexed, 5-8 $\times 4.2-6.5 \mathrm{~mm}$, apices rounded; lower lobe ovate or orbicular, not spreading or reflexed, 4.7-7 $\times 4.7-9 \mathrm{~mm}$, apex rounded. Stamens slightly or not exserted, fused in 2 pairs, filaments papillose; anterior filaments inserted at 10-12.7 $\mathrm{mm}$ from corolla base which is $48-51 \%$ of corolla length, filaments $14-15 \mathrm{~mm}$ long, anthers 1.9-2.2 $\times 0.9-1 \mathrm{~mm}$; posterior filaments inserted at 11.5-14.5 mm from corolla base which is $55-58 \%$ of corolla length, filaments $10-11 \mathrm{~mm}$ long, anthers $1.7-2.1 \times$ 0.9-1 mm; staminodes 1-1.2 mm long. Disk c. $0.9 \mathrm{~mm}$ high, a simple annular ring or 5-crenate. Pistil 16.5-22 mm long; stipe 1.5-4 mm long, glabrous or with few sessile glands; ovary 12.5-14.7 mm long, with sessile glands; style 2.5-3.3 mm long, glandular pubescent; stigma circular. Capsule 18-37 cm long, c. 4 mm wide. Seed grain $0.8-1.2 \times 0.2-0.3 \mathrm{~mm}$, papillose, bubble cells present at base of hilar appendage; apical appendage a filiform hair, c. $9 \mathrm{~mm}$ long; hilar appendage a single filiform hair, c. $10 \mathrm{~mm}$ long; appendages not papillose.

Distribution. Peninsular Malaysia (Johor, Pahang, Perak, Terengganu), Singapore, Sumatra. 


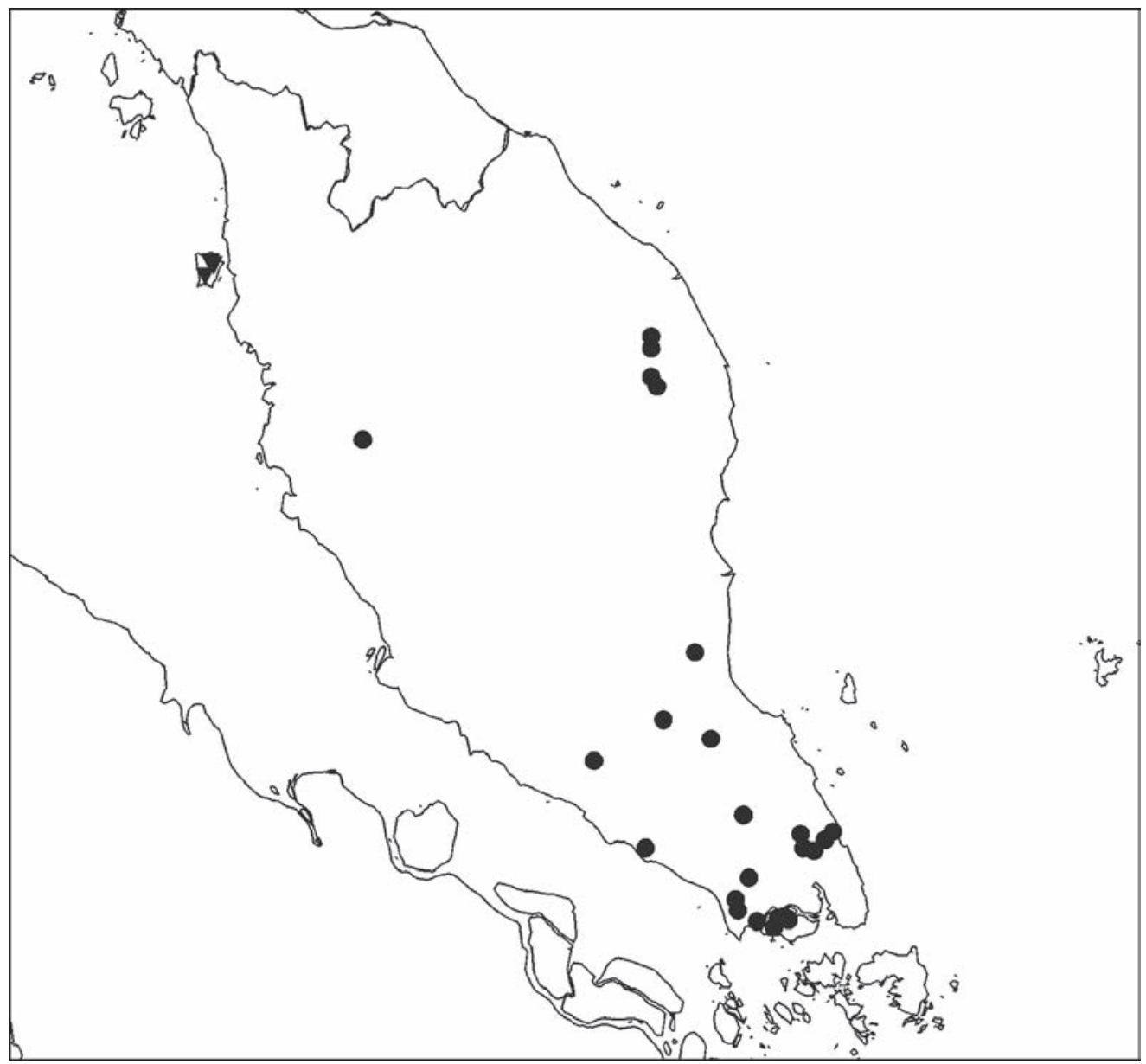

Fig. 19. Distribution of Aeschynanthus volubilis Jack ( $\boldsymbol{\nabla})$ in Peninsular Malaysia and Aeschynanthus wallichii R.Br. (•) in Singapore and Peninsular Malaysia.

Habitat and ecology. In lowland dipterocarp forest, or in peat swamp forest at 0-760 $\mathrm{m}$ altitude.

Provisional IUCN conservation assessment. Least Concern (LC). This species is relatively infrequently collected but has an EOO and AOO considerably above levels considered to be concerning. In Singapore it is considered to be Critically Endangered and is now confined to Nee Soon Swamp.

Additional Singaporean and Peninsular Malaysian specimens examined. PENINSULAR MALAYSIA: Johor: 5 miles S of Labis FR, 17 Apr 1966, Whitmore, T.C. KEP207 (KEP); Mawai-Sedili Road, 24 Jan 1961, Chew, W.-L. CWL222 (C, E, K, L, SING); Kuala Sedili fresh water swamp forest, 6 m, 26 May 1961, Burkill, H.M. HMB2668 (SING); Kota Tinggi, Kuala Sedili New Road, 23 Jun 1959, Kadim \& Noor, M. 138 (SING); ibidem, 23 Jun 1959, Kadim \& Noor, M. 131 (SING); ibidem, 25 Sep 1959, Shah, M. \& Noor, M. MS813 (E, L, 
SING); ibidem, 23 Jun 1959, Shah, M. 138 (K); Kota Tinggi, Gunung Panti West, 550 m, 19 Jul 1981, Maxwell, J.F. 81-182 (L, SING); Gunung Panti, 520 m, 5 Jul 1970, Samsuri Ahmad SA318 (SING); ibidem, 4 Mar 1987, Kiew, R. RK2398 (SING); ibidem, 470 m, 24 Aug 1986, Wong, K.M. s.n. (KEP); ibidem, 22 Jan 1994, Kiew, R. RK3775 (KEP); ibidem, 4 Mar 1987, Kiew, R. RK2398 (KEP); ibidem, 460 m, 31 Jul 2008, Yao, T.L., Lim, C.L., Rosdi, M. \& Ayau, K. FRI65386 (KEP); ibidem, 480 m, 5 Feb 1981, Collenette, I.S. 2254 (E); Pontian, Pengkalan Raja, 28 Jun 1939, Henderson, M.R. SFN36656 (K, SING); Gunung Ledang, Lobb, T. 111 (K); Alor Bukit, 22 Nov 1966, Hardial, S. 537 (K); Sungai Kayu, 12 Oct 1936, Kiah SFN32054 (BM, K, LAE, SING); Alor Bukit, 22 Nov 1966, Hardial, S. 537 (SING); Endau, Sg. Sempanong, 4 Feb 1986, Kiew, R. RK2112 (SING); Ulu Batu Pahat, Kampong Simpai, 1892, Lake \& Kelsall, H. s.n. (SING); Sebrau [?Tebrau], Aug 1908, Ridley, H.N. s.n. (SING); Tempayang River, 1908, Ridley, H.N. s.n. (BM, SING); Tanjong Kupang, Jun 1890, Ridley, H.N. s.n. (SING); Nov-Dec 1935-1935, Vesterdal, A. 306 (C); Kluang, 20 Nov 1922, Holttum, R.E. 9489 (SING); ibidem, 7 Mar 2000, Morgany, T., Tan, H.T.W. \& Loo, A.H.B. M260 (SINU);

Pahang: Pekan, Pekan Forest Reserve, Sungai Bebar, 21 Apr 2005, Zainon, A.S. FRI50105 (KEP); Rompin, Endau-Rompin State Park, Trail to Gunung Keriong, 20 Aug 2002, Sam, Y.Y. FRI47144 (KEP); Perak: Chenderiang, Gunong [Gunung] Bujang Melaka, 120 m, 10 Aug 1959, Mrs. Allen \& Kadim 436 (SING); Terengganu: Ulu Brang, 760 m, Jul 1937, Moysey, L. \& Kiah SFN33624 (LAE, SING); Sekayu, 7 May 1988, Kiew, R. RK2696 (KEP); Sakayu, 29 Aug 1986, Anthony, S. SA639 (KEP); Dungun, Pasir Raja Forest Reserve, Hutan Lipur Cemerung, 18 Oct 2002, Sam, Y.-Y. FRI47158 (KEP); ibidem, 18 Mar 1998, Anonymous s.n. (SING); Ulu Brang, Terengganu Tambahan FR, Sg. Chih, 360 m, 26 Mar 2013, Imin, K., Ong, P.T. \& Kueh, H.L. FRI77847 (KEP).

SINGAPORE: unknown loc., 1822, Wallich, N. 798 (CGE, K-W); ibidem, unknown s.n. (SING); Seletar, Gan, J.T.W.M., Hardie, J.A. \& Khng, Y.W.K. 1012 (SINU); Seletar, 16 May 1992, Gan, J.T.W.M., Hardie, J.A. \& Khng, Y.W.K. 1037 (SINU); Seletar, 2 Oct 1948, Sinclair, J. SFN38248 (SING); Seletar, 18 Sep 1948, Sinclair, J. SFN38246 (E, NY, P, SING); Jurong Road 15th mile, 19 Oct 1932, Corner, E.J.H. SFN26034 (K, SING); Nee Soon Swamp Forest, 26 Jan 1995, Karim, N.A. et al. NK206 (SING); ibidem, 5 m, 19 Mar 1982, Maxwell, J.F. 8280 (SING); Chan Chu Kang, Jun 1892, Ridley, H.N. s.n. (MEL); Chan Chu Kang, Jan 1892, Ridley, H.N. s.n. (SING); ibidem, 4 Apr 1890, Goodenough, J.S. s.n. (SING); Krangi [Kranji], 7 Dec 1889, Ridley, H.N. s.n. (SING); ibidem, 1894, Mat s.n. (SING); Mandai Road, 19 Feb 1931, Spare, G.H. F905 (K); Bukit Mandai, Sep 1890, Goodenough, J.S. s.n. (SING).

Notes. This species is most similar to Aeschynanthus obconicus but the flowers, and in particular the calyx, are smaller. The calyx is also green (vs red) and more saucershaped than cup-shaped.

\section{Further study necessary}

Rafidah, A.R. \& Nor Ezzawanis, A.T. FRI64274 (KEP) from G. Telapak Burok in Berembun Forest Reserve, Negeri Sembilan, Malaysia, has small opposite leaves, 0.9$2.1 \times 0.6-1.1 \mathrm{~cm}$, and seeds with one long appendage at each end. This combination of characters is otherwise unknown in Peninsular Malaysia and without flowers the identity of this plant remains a mystery. Aeschynanthus longiflorus, which has the same fruit and seed type, is known from the same locality but it is not known to 
have leaves anywhere near as small. FRI64274 is rather reminiscent of Aeschynanthus fruticosus from Sumatra in leaf shape and seed type but that species always has most leaves in whorls of three or more.

ACKNOWLEDGEMENTS: I thank Jana Leong-Škorničková (SING) for handling the review process of this paper and, particularly, for taking many photographs and compiling the plates for me. I thank Ali Ibrahim (SING), Preecha Karaket (BKF) and Saw Leng Guan (KEP) for additional photos. I also thank Ruth Kiew (KEP) for her assistance in locating a number of Malaysian place names; Martin Pullan (E) for both enabling the use of and assistance with the Padme database; and the Singapore Botanic Gardens' Herbarium curatorial staff for arranging loans. Ruth Kiew (KEP) and Gemma Bramley (K) are thanked for their useful comments on the manuscript.

\section{References}

Bhattacharyya, U.C. \& Goel, A.K. (2015). Systematic account of the genus Aeschynanthus Jack (Gesneriaceae) in India. Phytotaxonomy 14: 1-22.

Chong, K.Y., Tan, H.T.W. \& Corlett, R.T. (2009). A Checklist of the Total Vascular Plant Flora of Singapore, Native, Naturalised and Cultivated Species. Singapore: Raffles Museum of Biodiversity Research.

Clarke, C.B. (1883). Cyrtandreae. In: De Candolle, A. \& De Candolle, C. (eds) Monographiae phanerogamarum, vol. 5. Pp. 1-303, 32 pl. Paris: Masson.

Denduangboripant, J., Mendum, M. \& Cronk, Q.C.B. (2001). Evolution in Aeschynanthus (Gesneriaceae) inferred from ITS sequences. Pl. Syst. Evol. 228: 181-197.

IUCN (2012). IUCN Red List Categories and Criteria: Version 3.1. Second edition. Gland, Switzerland and Cambridge, UK: IUCN.

Kress, W.J., DeFilipps, R.A., Farr, E. \& Daw Yin Yin Kyi (2003). A checklist of the trees, shrubs, herbs and climbers of Myanmar. Contr. U.S. Natl. Herb. 45: 1-590.

Mendum, M. \& Atkins, H.J. (2003). The Gesneriaceae of Sulawesi I: an introduction. Edinburgh J. Bot. 60: 299-304.

Mendum, M., Lassnig, P., Weber, A. \& Christie, F. (2001). Testa and seed appendage morphology in Aeschynanthus (Gesneriaceae): phytogeographical patterns and taxonomic implications. Bot. J. Linn. Soc. 135: 195-213.

Middleton, D.J. (2007). A revision of Aeschynanthus (Gesneriaceae) in Thailand. Edinburgh J. Bot. 64: 363-429.

Middleton, D.J. (2009). A revision of Aeschynanthus (Gesneriaceae) in Cambodia, Laos and Vietnam. Edinburgh J. Bot. 66: 391-446.

Ridley, H.N. (1923). The Flora of the Malay Peninsula, vol. 2. London: L. Reeve \& Co., Ltd. (Gesneriaceae, Pp. 495-547)

Saw, L.G. (2010). Vegetation of Peninsular Malaysia. In: Kiew, R., Chung, R.C.K., Saw, L.G., Soepadmo, E. \& Boyce, P.C. (eds) Flora of Peninsular Malaysia 1: 21-46.

Thiers, B. (continuously updated). Index Herbariorum: A global directory of public herbaria and associated staff. New York Botanical Garden's Virtual Herbarium. http://sweetgum. nybg.org/science/ih/ (accessed on 12 Jan. 2016).

Tjitrosoedirdjo, S.S., Zakaria, R. \& Nurainas (2009). Notes on Aeschynanthus (Gesneriaceae) of Sumatra, Indonesia. Blumea 54: 278-279. 
Turner, I.M. (1993). The names used for Singapore plants since 1900. Gard. Bull. Singapore 45: 1-287.

Turner, I.M. (1997 ['1995']). A catalogue of the vascular plants of Malaya. Gard. Bull. Singapore 47: 1-757.

Wang, W.T., Pan, K.Y., Li, Z.Y., Weitzman, A.L. \& Skog, L.E. (1998). Gesneriaceae. In: Wu, Z.Y. \& Raven, P.H. (eds) Flora of China, vol. 18. Pp. 244-401. Beijing: Science Press; St. Louis: Missouri Botanical Garden Press.

Weber, A. (2004). Gesneriaceae. In: Kubitzki, K. \& Kadereit, J.W. (eds) The families and genera of vascular plants, vol. 7, Flowering plants: Dicotyledons; Lamiales (except Acanthaceae including Avicenniaceae). Pp 63-158. Berlin \& Heidelberg: Springer.

Williams, C. (2014). The rediscovery of a presumed nationally extinct Aeschynanthus. Gardenwise 43: 10-11.

Appendix 1. Index of Exsiccatae. The number in parentheses refers to the number of the taxon in the text.

Abbe, E.C. et al. 9119 (11); Abbe, L.B. et al. 9767 (11); Abdul Samat bin Abdullah 68 (11); Addison, G.H. s.n. (9); Allen, B.M. 4852A (10); Alvins, M.V. 1942 (10); Anderson, J.W. 95 (7), 156 (9); Annandale, N. \& Robinson, H.C. s.n. (10); Anonymous s.n. (14); Anthony, S. SA230 (3), SA231 (11), SA233 (9), SA481 (7), SA482 (9), SA639 (14), SA740 (10), SA897 (9); Anthonysamy, S. SA1155 (9); Asmah 22 (9); Avé, W. 165 (8); Aziz Budin 48644 (8).

Barnes, E. s.n. (3); Batten Pooll, A.H. s.n. (11); Beccari, O. 36 (8); Best, G.A. 21255 (6); Bogner 1699 (10); Bramley, G. \& Neale, S. GB31 (11); Bramley, G. \& Sam, Y.-Y. GB27 (9); Bremer, B. \& Bremer, K. 1808 (9); Burkill, H.M. HMB730 (11), HMB760 (11), HMB856 (9), HMB868 (3), HMB1996 (9), HMB2038 (9), HMB2665 (9), HMB2668 (14), HMB2811 (9), HMB2812 (7), HMB4221 (3), 8859 (7); Burkill, H.M. \& Holttum, R.E. s.n. (9), 7888 (7), 8418 (9), 8531 (11), 8549 (9); Burkill, H.M. \& Md Haniff 12542 (6), 12798 (8), 12833 (9), 12971 (9), 15750 (1), 17258 (9); Burkill, H.M. et al. HMB2430 (9); Burkill, I.H. 2159 (10), 6102 (9); Burn-Murdoch s.n. (11); Burtt, B.L. B1739 (6); Burtt, B.L. \& Woods, P.J.B. B1644 (7), B1739 (6), B1643 (9).

Carrick, J. 607 (9); Carter s.n. (9); Castle-Smith, P.M. 15 (9), 42 (11); Chan, K.Y. FRI49293 (9), FRI64737 (9); Chan, Y.M. FRI49298 (8); Chan, Y.M. \& Kiew, R. et al. FRI60504 (9); Chan, Y.M. et al. FRI70603 (8); Chew, M.Y. \& Rosdi, M. FRI55583 (9); Chew, M.Y. et al. FRI53648 (9); Chew, W.-L. CWL222 (14), CWL224 (9), CWL772 (11), CWL922 (11), CWL1213 (8); Chin, S.C. 165 (11), 233 (11), 1441 (9), 1812 (9); Chin, S.C. et al. 4533 (1); Choo, J.P.S. et al. PYL2 (9); Chua, L.S.L. FRI26700 (8), FRI39082 (9), FRI40785 (7); Chua, L.S.L. et al. FRI45633 (9); Collenette, I.S. 2253 (9), 2254 (14); Corner, E.J.H. s.n. (10), SFN26034 (14), s.n. (3); Corner, E.J.H. \& Henderson, M.R. SFN36611 (9), SFN36612 (9); Corporal s.n. (9); Cultivated C4877 (6), C7315 (11), C7431 (9), C7650 (8); Cuming, H. 2387 (10); Curtis, C. s.n. (11), 1311 (11), 1388 (8), 2142 (6), 2503 (9), 2990 (8), 3335 (8).

Darnaedi, D. et al. 566 (3); Davidson, C. 1292 (1); Davis 69248A (11), 69431 (9); Davison, G.W.H. s.n. (10); Derry, R. 205 (10), 305 (10); Ding Hou 657 (8); Dransfield, J. s.n. (11); Durnford, L. s.n. (9).

Ernst, A. 1189 (9); Evans, J.H.N. s.n. (9); Everard, B. \& Young, D. 94 (9); Everett, B. FRI13553 (9). Federated Malay States Museum Coll s.n. (11); Fielding, J. s.n. (9); Flippance, F. s.n. (13); Fox, W. s.n. (11); Franck, C.W. 209 (9), 1150 (10). 
Gadoh anak Umbai for A.H. Millard KL781 (10), KL1680 (10); Gadoh anak Umbai KL1319 (12); Gan, J.T.W.M. et al. 1012 (14), 1037 (14); Gerb 3257 (9); Goodenough, J.S. s.n. (9), 1518 (10), 1711 (9), 2705 (9), 2706 (9); Griffith, W. s.n. (10); Gwee, A.T. SING2010443 (9); Gwynne-Vaughan, D.T. 410 (10).

Hardial, S. 537 (14); Hardial, S. \& Samsuri Ahmad 18 (9), 290 (8); Haviland, G.D. s.n. (9); Hawkins, A.S.M. s.n. (12), 6 (11); Heaslett, E.A. s.n. (1); Henderson, M.R. s.n. (2), s.n. (11), s.n. (9), FMS11263 (9), 11365 (11), FMS11453 (9), 11713 (9), 11829 (11), SFN17921 (9), 17988 (11), SFN21928 (10), SFN23534 (11), 25065 (9), SFN29643 (9), SFN36656 (14); Heng, H.P. et al. HP10 (6), HP12 (9); Holttum, R.E. s.n. (7), 9489 (14), 9715 (10), 15312 (2), 20769 (11), 20772 (3), 21528 (7); Hons. Students 1 (9); Hose, G. 46 (11); Hume, H.L. 8253 (10), 8943 (10), 9228 (10), 9596 (1), 9772 (1).

Ibrahim, H. \& Lua, H.K. SING-2013-092 (9); Ibrahim, H. et al. SING2013-093 (9); Imin, K. \& Asmarayani, H.S. FRI76004 (8); Imin, K. \& Kueh, H.L. et al. FRI58561 (7); Imin, K. \& Syahida Emiza, S. FRI76065 (3), FRI76073 (3); Imin, K. et al. FRI58557 (9), FRI58570 (11), FRI58593 (4), FRI58594 (10), FRI66402 (9), FRI66493 (9), FRI68188 (8), FRI71615 (8), FRI71855 (9), FRI71954 (11), FRI77536 (3), FRI77847 (14).

Jaemat 25181 (9); Johnston, A.M. 76 (11); Jong, K. 9017 (1); Julius, A. FRI53313 (1), FRI54942 (9), FRI56020 (11), FRI57451 (8), FRI73605 (7); Julius, A. \& Imin, K. FRI56237 (10); Julius, A. et al. FRI53302 (11), FRI57479 (7), FRI57483 (11), FRI57691 (1), FRI57716 (9); Jumali K2077 (9); Jumali \& Heaslett, E.A. KJ732 (9); Jutta, M. \& Kueh, H.L. FRI59582 (10), FRI59583 (9).

Kadim \& Noor, M. 131 (14), 135 (9), 138 (14); Kalong 22423 (9); Kamarudin S. FRI34629 (9), FRI33755 (11); Kamarul Hisham, M. FRI52054 (11); Kamarul Hisham, M. et al. FRI52094 (3); Karim, N.A. et al. NK206 (14); Kassim, M. 550 (9); Kelsall, H. s.n. (12); Keng, H. \& Jumali K2077 (9); Keng, H. et al. K8010 (11), CTV86 (9), D-5 (11), 1 (10), 27 (9), 54 (9), 67 (9), 85 (7), 86 (9), 93 (9), 97 (9), 410 (11), 4755 (7); Khoo, $R$. \& Ng, S.M. 074 (9); Kiah SFN32054 (14), 23943 (8); Kiew, R. RK832 (9), RK1002 (9), RK1081 (9), RK1546 (11), RK1548 (9), RK2112 (14), RK2160 (9), RK2262 (8), RK2398 (14), RK2398 (14), RK2430 (11), RK2641 (9), RK2696 (14), RK2727 (9), RK3241 (3), RK3264 (9), RK3293 (7), RK3356 (9), RK3775 (14), RK4862 (5), RK4869 (9), RK5239 (8), RK5307 (3); Kiew, R. \& Anthony, S. RK3414 (9); Kiew, R. et al. FRI57497 (7); King's Collector 2012 (8), 2049 (8), 2849 (8), 3641 (8), 4738 (2), 7022 (7), 8314 (7), 10179 (8); Klackenberg, J. \& Lundin, R. 689 (11); Kloss, C.B. s.n. (11), 6476 (10); Kochummen, K.M. FRI2654 (9), FRI16215 (7), FRI16427 (8), FRI16516 (9), FRI16796 (1), FRI29126 (8), FRI29332 (6); Kunstler, H. 2636 (9), 4463 (1).

Lake \& Kelsall, H. s.n. (14); Latiff, A. 347 (1); Latiff, A. \& Zainudin, A. ALM1033 (10), ALM2764 (9), ALM3121 (11); Latiff, A. et al. ALM970 (11), ALM1644 (10), ALM1789 (7), ALM1813 (6); Lee, D.W. s.n. (11); Leong, P. SING2010-809 (9); Leong, P. et al. SING2012-165 (9); Lewis, G.P. 251 (9); Lim, C.L. FRI73033 (9); Lim, C.L. \& Kueh, H.L. FRI64886 (8); Lo, Y.N. \& Mahmud 83 (9), 183 (5); Lobb, T. 111 (14); Long, F.R. 13 (10), 14 (9); Low, Y.W. LYW140 (11); Lua, H.K. \& Ibrahim, H. SING2015-084 (1).

Mahammud 17178 (9); Mahmood KEP17216 (9); Mahmud bin Sidek s.n. (9), 4816 (11); Maingay, A.C. K.D.1219 (9), K.D.1218 (9); Mashall 35847 (10); Mat s.n. (14); Maxwell, J.F. 78-90 (9), 81-166 (9), 81-182 (14), 82-80 (14); Md Haniff s.n. (13), 21074 (8), 21088 (7); Md Haniff \& Md Nur 2314 (9), 2347 (3), 2456 (11), 2469 (3), 8046 (8), 10390 (10); Md Nur s.n. (2), 11056 (11), 11138 (9), SFN24637 (10), SFN32980 (3), SFN33963 (9), SFN34079 (9), SFN34254 (1), SFN34400 (9); Md Nur \& Foxworthy, F.W. 11963 (1); Md Nur \& Kiah 7783 (9); Mead, J.P. 27945 (11); Mendum, M. s.n. 
(9); Merton, L.F.H. 004194 (9); Mitchell s.n. (7); MK \& AR 1321 (1); Mohd Hairul, M.A. \& Mohd Nazri, A. et al. FRI69892 (11); Mohd Hairul, M.A. et al. FRI69938 (11), FRI69946 (7), FRI70936 (10), FRI72324 (1); Mohd Shah MS 3446 (11); Mohd Shah \& Mohd Ali MS 3046 (9); Mohd Shah \& Sidek MS 1073 (11), MS1110 (11); Mohd. Hairul, M.A. FRI60925 (8); Mohd. Kassim bin Rajab 488 (7); Morgany, T. et al. M260 (14); Moysey, L. \& Kiah SFN33624 (14); Mrs. Allen \& Kadim 436 (14).

Napier, W. s.n. (12); Ng, F.S.P. FRI1136 (9), FRI5813 (8), FRI5931 (11), FRI27146 (11); Ng, H.H. \& Lok, A.F.S.L. s.n. (1); Nongchi s.n. (9), 8 (1); Noor, M. MN.1913 (9); Norain, Raihana \& Rosjana 07 (12).

Okada, H. et al. 1027 (11), 1050 (3).

Perumal, B. et al. FRI41512 (7), FRI41602 (11), FRI41631 (3), FRI41661 (11); Phoon, S.N. FRI51987 (9), FRI60496 (11); Phoon, S.N. \& Imin, K. FRI60648 (7); Phoon, S.N. \& O’Byrne, P. et al. FRI60557 (11); Phoon, S.N. et al. FRI51576 (9); Poore, M.E.D. 466 (11), 1028 (11), 1064 (8); Purseglove, J.W. P4202 (9), P4182 (9), P4164 (7), P4222 (3). Quaife s.n. (7).

Rafidah, A.R. \& Nor Ezzawanis, A.T. FRI64274 (1); Rafidah, A.R. et al. FRI55631 (9); Rahimatsah Amat $N 12$ (9); Rao \& et al. K8010 (11), 167 (9); Ridley, H.N. s.n. (1), 1578 (9), 1700 (6), 2151 (1), 2167 (7), 2704 (10), 2706 (9), 2710 (9), 2905 (8), 5513 (9), 6244 (1), 11447 (7), 13372 (8), 13599 (4), 13600 (11), 13603 (7), 14063 (8), 14280 (6), 14281 (10), 14282 (10), 16090 (11), 16122 (3); Ridley, H.N. \& Curtis, C. 7365 (3); Robinson, H.C. s.n. (7); Robinson, H.C. \& Kloss, C.B. 5997 (9), 6049 (9); Rosdi, M. \& Phoon, S.N. et al. FRI59867 (11), FRI59872 (3); Rosdi, M. \& Rafidah, A.R. FRI59837 (9); Rosdi, M. et al. FRI58753 (9), FRI66272 (7), FRI66336 (10); Rostado 64 (9).

Sabari, D. FRI32715 (11); Sam, Y.-Y. FRI44492 (10), FRI46549 (10), FRI46602 (9), FRI47104 (10), FRI47144 (14), FRI47158 (14), FRI47208 (11); Sam, Y.-Y. et al. FRI47150 (9); Samsuri Ahmad SA96 (11), 309 (9), S.311 (9), SA318 (14), SA1125 (9); Saw, L.G. FRI34368 (11), FRI36398 (11), FRI37667 (7), FRI37684 (10); Saw, L.G. \& Mohd Hairul, M.A. FRI48326 (1); Saw, L.G. \& Mustafa, D. FRI37514 (9); Saw, L.G. et al. FRI48241 (11); Scortechini, B. 36a (9), $46 a$ (8), s.n. (11), $468 b$ (11), 35 (6), 37 (10), 39 (9), 291 (9), 330 (10), 388 (11), 1815 (1); Seimund, E. s.n. (9), 390 (10), 459 (8); Shah, M. 138 (14), 155 (1); Shah, M. \& Mahmud MS4895 (9), MS4978 (9); Shah, M. \& Noor, M. MS607 (9), MS813 (14); Shah, M. \& Sidek MS1064 (9); Shah, M. et al. MS3407 (8); Sidek bin Kiah S.277 (8), SK515 (8); Siew Wei Hoe 47 (11); Sinclair, J. s.n. (10), 5090 (10), 5376 (9), 6198 (7), 8151 (10), 9949 (11), 10568 (9), SFN38246 (14), SFN38248 (14), SFN38609 (9), SFN40358 (10); Sinclair, J. \& Kiah SFN38670 (11), SFN38800 (7), SFN38805 (8); Smith, G. 443 (7), 448 (9), 513 (9); Smith, J.W. 63690 (3); Spare, G.H. F905 (14), 991 (9), 2018 (9), 2047 (11), 2114 (7), 2139 (9), SFN36226 (8); Staples, G. et al. SING2009-157 (9); Stone, B.C.M. s.n. (8), 5889 (9), 6543 (9), 7194 (11), 7218 (11), 9566 (8), 10798 (9), 11091 (8), 12758 (5), 13764 (9), 14045 (11), 15071 (11), 15134 (9), 15167 (8), 15366 (11), 15497 (11); Stone, B.C.M. \& Badaruddin 12068 (8); Stone, B.C.M. \& Mahmud 8500 (9); Strugnell, E.J. 10515 (8); Syahida Emiza, S. FRI51460 (11), FRI57293 (11); Syahida Emiza, S. \& Angan, A. FRI55108 (9); Syahida Emiza, S. \& Chew, M.Y. FRI57277 (3); Symington, C.F. 20938 (3), 21390 (8), 36073 (9), 51762 (11), 56711 (11).

Tam Sheh May TSM2 (11); Tan, W.K. et al. TWK1 (11), TWK12 (9); Tay, E.P. 125 (6); Teruya, Z. 951 (1); Togashi, M. s.n. (11), 622111 (11).

UNESCO limestone expedition 440A (10), 239 (9); unknown FMS17068 (8), KEP78844 (9), KEP93108 (12), s.n. (11), K6502 (9), s.n. (7), 4636 (9). 
Van Balgooy, M.M.J. 2118 (8), 2154 (11), 2658 (3), 2663 (11), 7133 (11), 7149 (3), 7228 (11). Vesterdal, A. 306 (14).

Wallich, N. 798 (14); Weber, A. s.n. (11); Whitmore, T.C. KEP207 (14), FRI227 (9), FRI3887 (11), FRI12601 (7), FRI15450 (11), FRI15495 (11), FRI20392 (8); Wilkie, P. et al. FRI52906 (11), FRI75005 (11); Wong, K.M. FRI35244 (11), s.n. (14); Wong, Y.K. KEP93272 (8); Woods, P.J.B. 616 (3), 632 (11), 634 (3), 680 (11); Woods, P.J.B. et al. 615 (11); Worthington, R.D. 12463 (11), 13326 (9); Wray, L. s.n. (11), 149 (8), 625 (7), 655 (9), 656 (11), 873 (9), 1636 (11), 1772 (8), 3217 (9), 4245 (1); Wray, L. \& Robinson, H.C. 5483 (3); Wyatt-Smith, J. KEP 56945 (11), 78808 (11).

Yao, T.L. FRI65302 (3), FRI57961 (6); Yao, T.L. et al. FRI55845 (11), FRI55898 (11), FRI57913 (10), FRI65386 (14), FRI65387 (9), FRI65495 (7), FRI77328 (9), FRI77335 (7); Yapp, R.H. 161 (1), 440 (11), 547 (8), 610 (12); Year III students FSC312 (9); Yeo, C.K. s.n. (9); Yong KEP99330 (9).

Zainal Mustafa ZM64 (9); Zainon, A.S. FRI50105 (14); Zainudin, A. AZ40 (9); Zainudin, A. et al. AZ2438 (9). 
